\title{
KLOOSTERMAN SUMS AND MAASS CUSP FORMS OF HALF INTEGRAL WEIGHT FOR THE MODULAR GROUP
}

\author{
SCOTT AHLGREN AND NICKOLAS ANDERSEN
}

Abstract. We estimate the sums

$$
\sum_{c \leq x} \frac{S(m, n, c, \chi)}{c}
$$

where the $S(m, n, c, \chi)$ are Kloosterman sums of half-integral weight on the modular group. Our estimates are uniform in $m, n$, and $x$ in analogy with Sarnak and Tsimerman's improvement of Kuznetsov's bound for the ordinary Kloosterman sums. Among other things this requires us to develop mean value estimates for coefficients of Maass cusp forms of weight $1 / 2$ and uniform estimates for $K$-Bessel integral transforms. As an application, we obtain an improved estimate for the classical problem of estimating the size of the error term in Rademacher's formula for the partition function $p(n)$.

\section{Contents}

1. Introduction and statement of results

2. Background

3. A mean value estimate for coefficients of Maass cusp forms

4. The Kuznetsov trace formula

5. A theta lift for Maass cusp forms

6. Estimates for a $K$-Bessel transform

7. Application to sums of Kloosterman sums

8. A second estimate for coefficients of Maass cusp forms

9. Sums of Kloosterman sums in the case $m=1$

10. Application to the remainder term in Rademacher's formula

\section{InTRODUCTION AND STATEMENT OF RESUlts}

The Kloosterman sum

$$
S(m, n, c)=\sum_{\substack{d \bmod c \\(d, c)=1}} e\left(\frac{m \bar{d}+n d}{c}\right), \quad e(x):=e^{2 \pi i x}
$$

plays a leading part in analytic number theory. Indeed, many problems can be reduced to estimates for sums of Kloosterman sums (see, for example, [HB] or [Sa]).

Date: November 25, 2015.

The first author was supported by a grant from the Simons Foundation (\#208525 to Scott Ahlgren). 
In this paper we study sums of generalized Kloosterman sums attached to a multiplier system of weight $1 / 2$ (the sums $S(m, n, c)$ are attached to the trivial integral weight multiplier system). Kloosterman sums with general multipliers have been studied by Bruggeman [Br2], Goldfeld-Sarnak [GS], and Pribitkin [Pr], among others. We focus on the multiplier system $\chi$ for the Dedekind eta function and the associated Kloosterman sums

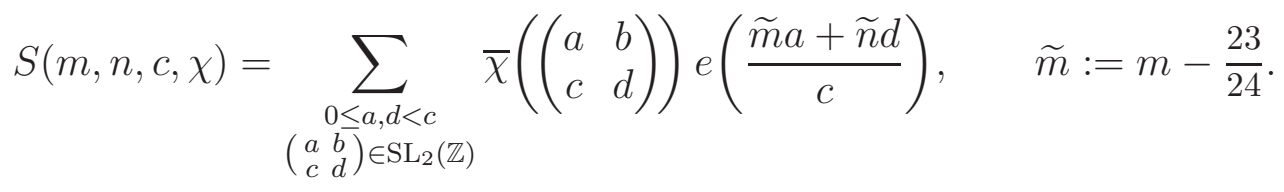

These sums are intimately connected to the partition function $p(n)$, and we begin by discussing an application of our main theorem to a classical problem.

In the first of countless important applications of the circle method, Hardy and Ramanujan [HR proved the asymptotic formula

$$
p(n) \sim \frac{1}{4 \sqrt{3} n} e^{\pi \sqrt{2 n / 3}}
$$

(and in fact developed an asymptotic series for $p(n)$ ). Perfecting their method, Rademacher [R1, R2] proved that

$$
p(n)=\frac{2 \pi}{(24 n-1)^{\frac{3}{4}}} \sum_{c=1}^{\infty} \frac{A_{c}(n)}{c} I_{\frac{3}{2}}\left(\frac{\pi \sqrt{24 n-1}}{6 c}\right),
$$

where $I_{\frac{3}{2}}$ is the $I$-Bessel function,

$$
A_{c}(n):=\sum_{\substack{d \bmod c \\(d, c)=1}} e^{\pi i s(d, c)} e\left(-\frac{d n}{c}\right)
$$

and $s(d, c)$ is a Dedekind sum (see (2.8) below). The Kloosterman sum $A_{c}(n)$ is a special case of (1.1); in particular we have (see \$2.8 below)

$$
A_{c}(n)=\sqrt{-i} S(1,1-n, c, \chi) .
$$

The series (1.2) converges very rapidly. For example, the first four terms give

$$
\begin{aligned}
p(100) & \approx 190568944.783+348.872-2.598+0.685 \\
& =190569291.742,
\end{aligned}
$$

while the actual value is $p(100)=190569292$.

A natural problem is to estimate the error which results from truncating the series (1.2) after the $N$ th term, or in other words to estimate the quantity $R(n, N)$ defined by

$$
p(n)=\frac{2 \pi}{(24 n-1)^{\frac{3}{4}}} \sum_{c=1}^{N} \frac{A_{c}(n)}{c} I_{\frac{3}{2}}\left(\frac{\pi \sqrt{24 n-1}}{6 c}\right)+R(n, N) .
$$

Since $I_{\nu}(x)$ grows exponentially as $x \rightarrow \infty$, one must assume that $N \gg \sqrt{n}$ in order to obtain reasonable estimates. For $\alpha>0$, Rademacher [R1, (8.1)] showed that

$$
R(n, \alpha \sqrt{n}) \ll_{\alpha} n^{-\frac{1}{4}}
$$

Lehmer [L1, Theorem 8] proved the sharp Weil-type bound

$$
\left|A_{c}(n)\right|<2^{\omega_{o}(c)} \sqrt{c},
$$


where $\omega_{o}(c)$ is the number of distinct odd primes dividing $c$. Shortly thereafter [L2] he used this bound to prove that

$$
R(n, \alpha \sqrt{n}) \ll_{\alpha} n^{-\frac{1}{2}} \log n .
$$

In 1938, values of $p(n)$ had been tabulated only for $n \leq 600$. Using (1.4), Lehmer showed [L1, Theorem 13] that $p(n)$ is the nearest integer to the series (1.2) truncated at $\frac{2}{3} \sqrt{n}$ for all $n>600$.

Building on work of Selberg and Whiteman [Wh], Rademacher [R3] later simplified Lehmer's treatment of the sums $A_{c}(n)$. Rademacher's book [R4, Chapter IV] gives a relatively simple derivation of the error bound $n^{-\frac{3}{8}}$ using these ideas.

Using equidistribution results for Heegner points on the modular curve $X_{0}(6)$, Folsom and Masri [FM] improved Lehmer's estimate. They proved that if $24 n-1$ is squarefree, then

$$
R\left(n, \sqrt{\frac{n}{6}}\right) \ll n^{-\frac{1}{2}-\delta} \quad \text { for some } \delta>0 .
$$

Using the estimates for sums of Kloosterman sums below, we obtain an improvement in the exponent, and we (basically) remove the assumption that $24 n-1$ is squarefree. For simplicity in stating the results, we will assume that

$$
24 n-1 \text { is not divisible by } 5^{4} \text { or } 7^{4} \text {. }
$$

As the proof will show (c.f. Section 8) the exponent 4 in (1.6) can be replaced by any positive integer $m$; such a change would have the effect of changing the implied constants in (1.7) and (1.8).

Theorem 1.1. Suppose that $\alpha>0$. For $n \geq 1$ satisfying (1.6) we have

$$
R\left(n, \alpha n^{\frac{1}{2}}\right) \ll_{\alpha, \epsilon} n^{-\frac{1}{2}-\frac{1}{168}+\epsilon} .
$$

In fact, our method optimizes when $N$ is slightly larger with respect to $n$.

Theorem 1.2. Suppose that $\alpha>0$. For $n \geq 1$ satisfying (1.6) we have

$$
R\left(n, \alpha n^{\frac{1}{2}+\frac{5}{252}}\right) \ll_{\alpha, \epsilon} n^{-\frac{1}{2}-\frac{1}{28}+\epsilon} .
$$

Remark. Suppose, for example, that $24 n-1$ is divisible by $5^{4}$. Then $n$ has the form $n=$ $625 m+599$, so that $p(n) \equiv 0(\bmod 625)$ by work of Ramanujan [BO, Section 22]. A sharp estimate for $R(n, N)$ for such $n$ is less important since $p(n)$ can be determined by showing that $|R(n, N)|<312.5$. The situation is similar for any power of 5 or 7 .

Remark. The estimates in Theorems 1.1 and 1.2 depend on progress toward the RamanujanLindelöf conjecture for coefficients of certain Maass cusp forms of weight $1 / 2$. Assuming the conjecture, we can use the present methods to prove

$$
R\left(n, \alpha n^{\frac{1}{2}}\right) \ll_{\alpha, \epsilon} n^{-\frac{1}{2}-\frac{1}{16}+\epsilon} .
$$

The analogue of Linnik and Selberg's conjecture ((1.10) below) would give

$$
R\left(n, \alpha n^{\frac{1}{2}}\right) \ll_{\alpha, \epsilon} n^{-\frac{3}{4}+\epsilon} .
$$

Computations suggest that this bound would be optimal. 
Theorems 1.1 and 1.2 follow from estimates for weighted sums of the Kloosterman sums (1.1). For the ordinary Kloosterman sum $S(m, n, c)$, Linnik [Li] and Selberg [Se2] conjectured that there should be considerable cancellation in the sums

$$
\sum_{c \leq x} \frac{S(m, n, c)}{c} .
$$

Sarnak and Tsimerman [ST] studied these weighted sums for varying $m, n$ and put forth the following modification of Linnik and Selberg's conjecture with an " $\epsilon$-safety valve" in $m, n$ :

$$
\sum_{c \leq x} \frac{S(m, n, c)}{c} \ll_{\epsilon}(|m n| x)^{\epsilon}
$$

Using the Weil bound [W]

$$
|S(m, n, c)| \leq \tau(c)(m, n, c)^{\frac{1}{2}} \sqrt{c},
$$

where $\tau(c)$ is the number of divisors of $c$, one obtains

$$
\sum_{c \leq x} \frac{S(m, n, c)}{c} \ll \tau((m, n)) x^{\frac{1}{2}} \log x
$$

Thus the conjecture (1.10) represents full square-root cancellation.

The best current bound in the $x$-aspect for the sums (1.9) was obtained by Kuznetsov $[\mathrm{Ku}$, who proved for $m, n>0$ that

$$
\sum_{c \leq x} \frac{S(m, n, c)}{c} \ll_{m, n} x^{\frac{1}{6}}(\log x)^{\frac{1}{3}} .
$$

Recently, Sarnak and Tsimerman [ST] refined Kuznetsov's method, making the dependence on $m$ and $n$ explicit. They proved that for $m, n>0$ we have

$$
\sum_{c \leq x} \frac{S(m, n, c)}{c} \ll_{\epsilon}\left(x^{\frac{1}{6}}+(m n)^{\frac{1}{6}}+(m+n)^{\frac{1}{8}}(m n)^{\frac{\theta}{2}}\right)(m n x)^{\epsilon},
$$

where $\theta$ is an admissible exponent toward the Ramanujan-Petersson conjecture for coefficients of weight 0 Maass cusp forms. By work of Kim and Sarnak [Ki, Appendix 2], the exponent $\theta=7 / 64$ is available. A generalization of their results to sums taken over $c$ which are divisible by a fixed integer $q$ is given by Ganguly and Sengupta GSe.

We study sums of the Kloosterman sums $S(m, n, c, \chi)$ defined in (1.1). In view of (1.3) we consider the case when $m$ and $n$ have mixed sign (as will be seen in the proof, this brings up a number of difficulties which are not present in the case when $m, n>0$ ).

Theorem 1.3. For $m>0, n<0$ we have

$$
\sum_{c \leq x} \frac{S(m, n, c, \chi)}{c} \ll_{\epsilon}\left(x^{\frac{1}{6}}+|m n|^{\frac{1}{4}}\right)|m n|^{\epsilon} \log x .
$$

While Theorem 1.3 matches [ST] in the $x$ aspect, it falls short in the $m n$ aspect. As we discuss in more detail below, this is due to the unsatisfactory Hecke theory in halfintegral weight. This bound is also insufficient to improve the estimate (1.5) for $R(n, N)$; for this we sacrifice the bound in the $x$-aspect for an improvement in the $n$-aspect. The resulting theorem, which for convenience we state in terms of $A_{c}(n)$, leads to the estimates of Theorems 1.1 and 1.2 . 
Theorem 1.4. Fix $0<\delta<1 / 2$. Suppose that $n \geq 1$ satisfies (1.6). Then

$$
\sum_{c \leq x} \frac{A_{c}(n)}{c} \ll_{\delta, \epsilon} n^{\frac{1}{4}-\frac{1}{56}+\epsilon} x^{\frac{3}{4} \delta}+\left(n^{\frac{1}{4}-\frac{1}{168}+\epsilon}+x^{\frac{1}{2}-\delta}\right) \log x .
$$

As in [Ku] and [ST], the basic tool is a version of Kuznetsov's trace formula. This relates sums of the Kloosterman sums $S(m, n, c, \chi)$ weighted by a suitable test function to sums involving Fourier coefficients of Maass cusp forms of weight 1/2 and multiplier $\chi$. Proskurin [P2] proved such a formula for general weight and multiplier when $m n>0$; in Section 4 we give a proof in the case $m n<0$ (Blomer [B] has recorded this formula for twists of the theta-multiplier by a Dirichlet character).

The Maass cusp forms of interest are functions which transform like $\operatorname{Im}(\tau)^{\frac{1}{4}} \eta(\tau)$, where

$$
\eta(\tau)=e\left(\frac{\tau}{24}\right) \prod_{n=1}^{\infty}(1-e(n \tau)), \quad \operatorname{Im}(\tau)>0
$$

is the Dedekind eta function, and which are eigenfunctions of the weight $1 / 2$ Laplacian $\Delta_{1 / 2}$ (see Section 2 for details). To each Maass cusp form $F$ we attach an eigenvalue $\lambda$ and a spectral parameter $r$ which are defined via

$$
\Delta_{\frac{1}{2}} F+\lambda F=0
$$

and

$$
\lambda=\frac{1}{4}+r^{2}
$$

We denote the space spanned by these Maass cusp forms by $\mathcal{S}_{\frac{1}{2}}(1, \chi)$. Denote by $\left\{u_{j}(\tau)\right\}$ an orthonormal basis of Maass cusp forms for this space, and let $r_{j}$ denote the spectral parameter attached to each $u_{j}$. Then (recalling the notation $\tilde{n}=n-\frac{23}{24}$ ), $u_{j}$ has a Fourier expansion of the form

$$
u_{j}(\tau)=\sum_{n \neq 0} \rho_{j}(n) W_{\frac{\operatorname{sgn}(n)}{4}, i r_{j}}(4 \pi|\widetilde{n}| y) e(\widetilde{n} x),
$$

where $W_{\kappa, \mu}(y)$ is the $W$-Whittaker function (see \$2.3). In Section 4 we will prove that

$$
\sum_{c>0} \frac{S(m, n, c, \chi)}{c} \phi\left(\frac{4 \pi \sqrt{\widetilde{m}|\widetilde{n}|}}{c}\right)=8 \sqrt{i} \sqrt{\widetilde{m}|\widetilde{n}|} \sum_{r_{j}} \frac{\overline{\rho_{j}(m)} \rho_{j}(n)}{\operatorname{ch} \pi r_{j}} \check{\phi}\left(r_{j}\right),
$$

where $\phi$ is a suitable test function and $\check{\phi}$ is the $K$-Bessel transform

$$
\check{\phi}(r):=\operatorname{ch} \pi r \int_{0}^{\infty} K_{2 i r}(u) \phi(u) \frac{d u}{u} .
$$

In Section 5 we introduce theta lifts (as in Niwa [N], Shintani [Sh]) which give a Shimuratype correspondence

$$
\mathcal{S}_{\frac{1}{2}}(N, \psi \chi, r) \rightarrow \mathcal{S}_{0}\left(6 N, \psi^{2}, 2 r\right)
$$

Here $\mathcal{S}_{k}(N, \nu, r)$ denotes the space of Maass cusp forms of weight $k$ on $\Gamma_{0}(N)$ with multiplier $\nu$ and spectral parameter $r$. These lifts commute with the action of the Hecke operators on the respective spaces, and in particular allow us to control the size of the weight $1 / 2$ Hecke eigenvalues, which becomes important in Section 8. The existence of these lifts shows that there are no small eigenvalues; this fact is used in Sections 6, 7, and 9. In particular, from (1.15) it will follow that if $\mathcal{S}_{1 / 2}(1, \chi, r) \neq 0$ then either $r=i / 4$ or $r>1.9$. 
To estimate the right-hand side of (1.13), we first obtain a mean value estimate for the sums

$$
|\widetilde{n}| \sum_{0<r_{j} \leq x} \frac{\left|\rho_{j}(n)\right|^{2}}{\operatorname{ch} \pi r_{j}} .
$$

In analogy with Kuznetsov's result [Ku, Theorem 6] in weight 0, we prove in Section 3 a general mean value result for weight $\pm 1 / 2$ Maass cusp forms which has the following as a corollary.

Theorem 1.5. With notation as above, we have

$$
|\widetilde{n}| \sum_{0<r_{j} \leq x} \frac{\left|\rho_{j}(n)\right|^{2}}{\operatorname{ch} \pi r_{j}}= \begin{cases}\frac{x^{\frac{5}{2}}}{5 \pi^{2}}+O_{\epsilon}\left(x^{\frac{3}{2}} \log x+|n|^{\frac{1}{2}+\epsilon} x^{\frac{1}{2}}\right) & \text { if } n<0 \\ \frac{x^{\frac{3}{2}}}{3 \pi^{2}}+O_{\epsilon}\left(x^{\frac{1}{2}} \log x+n^{\frac{1}{2}+\epsilon}\right) & \text { if } n>0 .\end{cases}
$$

We then require uniform estimates for the Bessel transform (1.14), which are made subtle by the oscillatory nature of $K_{i r}(x)$ for small $x$ and by the transitional range of the $K$-Bessel function. In Section 6 we obtain estimates for $\check{\phi}(r)$ which, together with Theorem [1.5, suffice to prove Theorem 1.3 .

To prove Theorem 1.4 we require a second estimate for the Fourier coefficients $\rho_{j}(n)$. In [ST] such an estimate is obtained via the simple relationship

$$
a(n)=\lambda(n) a(1)
$$

satisfied by the coefficients $a(n)$ of a Hecke eigenform with eigenvalue $\lambda(n)$. This relationship is not available in half-integral weight (the best which one can do is to relate coefficients of index $m^{2} n$ to those of index $n$ ). As a substitute, we employ an average version of a theorem of Duke [D].

Duke proved that if $a(n)$ is the $n$-th coefficient of a normalized Maass cusp form in $\mathcal{S}_{1 / 2}\left(N,\left(\frac{D}{\bullet}\right) \nu_{\theta}, r\right)\left(\nu_{\theta}\right.$ is the theta-multiplier defined in 92.2$)$ then for squarefree $n$ we have

$$
|a(n)| \ll_{\epsilon}|n|^{-\frac{2}{7}+\epsilon}|r|^{\frac{5}{2}-\frac{\operatorname{sgn}(n)}{4}} \operatorname{ch}\left(\frac{\pi r}{2}\right) .
$$

This is not strong enough in the $r$-aspect for our purposes (Baruch and Mao [BM have obtained a bound which is stronger in the $n$-aspect, but even weaker in the $r$-aspect).

Here we modify Duke's argument to obtain an average version of his result. We prove in Theorem 8.1 that if the $u_{j}$ as above are eigenforms of the Hecke operators $T\left(p^{2}\right), p \nmid 6$, then we have

$$
\sum_{0<r_{j} \leq x} \frac{\left|\rho_{j}(n)\right|^{2}}{\operatorname{ch} \pi r_{j}} \ll_{\epsilon}|n|^{-\frac{4}{7}+\epsilon} x^{5-\frac{\operatorname{sgn} n}{2}},
$$

for any collection of values of $n$ such that $24 n-23$ is not divisible by arbitrarily large powers of 5 or 7 . In Section 9 we use Theorem 8.1 and a modification of the argument of [ST] to prove Theorem 1.4. Section 10 contains the proofs of Theorems 1.1 and 1.2 .

Notation. Throughout, $\epsilon$ denotes an arbitrarily small positive number whose value is allowed to change with each occurence. Implied constants in any equation which contains $\epsilon$ are allowed to depend on $\epsilon$. For all other parameters, we will use a subscript (e.g. $\left.\ll_{a, b, c}\right)$ to signify dependencies in the implied constant. 


\section{ACKNOWLEDGMENTS}

We thank Andrew Booker for providing the computations which appear in Section 5 ,

\section{BACKGROUND}

We give some background on Maass forms and Kloosterman sums of general weight $k$ and multiplier $\nu$ (references for this material are [DFI, Sa, P2] along with the original sources [M1, M2, Ro2, Se1, Se2]). In the body of the paper we will work primarily in integral or half-integral weight and with the multiplier attached to a Dirichlet character or to the Dedekind eta-function, but for much of the background it is no more complicated to describe the general case. For the benefit of the reader we have attempted to provide a relatively self-contained account by providing details at various points.

2.1. Eigenfunctions of the Laplacian. Let $k$ be a real number and let $\mathbb{H}$ denote the upper half-plane. The group $\mathrm{SL}_{2}(\mathbb{R})$ acts on $\tau=x+i y \in \mathbb{H}$ via linear fractional transformations

$$
\gamma \tau:=\frac{a \tau+b}{c \tau+d}, \quad \gamma=\left(\begin{array}{ll}
a & b \\
c & d
\end{array}\right) .
$$

For $\gamma \in \mathrm{SL}_{2}(\mathbb{R})$ we define the weight $k$ slash operator by

$$
\left.f\right|_{k} \gamma:=j(\gamma, \tau)^{-k} f(\gamma \tau), \quad j(\gamma, \tau):=\frac{c \tau+d}{|c \tau+d|}=e^{i \arg (c \tau+d)},
$$

where we always choose the argument in $(-\pi, \pi]$.

The weight $k$ Laplacian

$$
\Delta_{k}:=y^{2}\left(\frac{\partial^{2}}{\partial x^{2}}+\frac{\partial^{2}}{\partial y^{2}}\right)-i k y \frac{\partial}{\partial x}
$$

commutes with the weight $k$ slash operator for every $\gamma \in \mathrm{SL}_{2}(\mathbb{R})$. The operator $\Delta_{k}$ can be written as

$$
\begin{aligned}
& \Delta_{k}=-R_{k-2} L_{k}-\frac{k}{2}\left(1-\frac{k}{2}\right), \\
& \Delta_{k}=-L_{k+2} R_{k}+\frac{k}{2}\left(1+\frac{k}{2}\right),
\end{aligned}
$$

where $R_{k}$ is the Maass raising operator

$$
R_{k}:=\frac{k}{2}+2 i y \frac{\partial}{\partial \tau}=\frac{k}{2}+i y\left(\frac{\partial}{\partial x}-i \frac{\partial}{\partial y}\right)
$$

and $L_{k}$ is the Maass lowering operator

$$
L_{k}:=\frac{k}{2}+2 i y \frac{\partial}{\partial \bar{\tau}}=\frac{k}{2}+i y\left(\frac{\partial}{\partial x}+i \frac{\partial}{\partial y}\right) .
$$

These raise and lower the weight by 2 : we have

$$
R_{k}\left(\left.f\right|_{k} \gamma\right)=\left.\left(R_{k} f\right)\right|_{k+2} \gamma \quad \text { and } \quad L_{k}\left(\left.f\right|_{k} \gamma\right)=\left.\left(L_{k} f\right)\right|_{k-2} \gamma .
$$

From (2.1) and (2.2) we obtain the relations

$$
R_{k} \Delta_{k}=\Delta_{k+2} R_{k} \quad \text { and } \quad L_{k} \Delta_{k}=\Delta_{k-2} L_{k} .
$$

A real analytic function $f: \mathbb{H} \rightarrow \mathbb{C}$ is an eigenfunction of $\Delta_{k}$ with eigenvalue $\lambda$ if

$$
\Delta_{k} f+\lambda f=0 .
$$


If $f$ satisfies (2.4) then for notational convenience we write

$$
\lambda=\frac{1}{4}+r^{2},
$$

and we refer to $r$ as the spectral parameter of $f$. From (2.3) it follows that if $f$ is an eigenfunction of $\Delta_{k}$ with eigenvalue $\lambda$ then $R_{k} f$ (resp. $L_{k} f$ ) is an eigenfunction of $\Delta_{k+2}$ (resp. $\Delta_{k-2}$ ) with eigenvalue $\lambda$.

2.2. Multiplier systems. Let $\Gamma=\Gamma_{0}(N)$ for some $N \geq 1$. We say that $\nu: \Gamma \rightarrow \mathbb{C}^{\times}$is a multiplier system of weight $k$ if

(i) $|\nu|=1$,

(ii) $\nu(-I)=e^{-\pi i k}$, and

(iii) $\nu\left(\gamma_{1} \gamma_{2}\right) j\left(\gamma_{1} \gamma_{2}, \tau\right)^{k}=\nu\left(\gamma_{1}\right) \nu\left(\gamma_{2}\right) j\left(\gamma_{2}, \tau\right)^{k} j\left(\gamma_{1}, \gamma_{2} \tau\right)^{k}$ for all $\gamma_{1}, \gamma_{2} \in \Gamma$.

If $\nu$ is a multiplier system of weight $k$, then it is also a multiplier system of weight $k^{\prime}$ for any $k^{\prime} \equiv k(\bmod 2)$, and the conjugate $\bar{\nu}$ is a multiplier system of weight $-k$. If $\nu_{1}$ and $\nu_{2}$ are multiplier systems of weights $k_{1}$ and $k_{2}$ for the same group $\Gamma$, then their product $\nu_{1} \nu_{2}$ is a multiplier system of weight $k_{1}+k_{2}$ for $\Gamma$.

When $k$ is an integer, a multiplier system of weight $k$ for $\Gamma$ is simply a character of $\Gamma$ which satisfies $\nu(-I)=e^{-\pi i k}$. If $\psi$ is an even (resp. odd) Dirichlet character modulo $N$, we can extend $\psi$ to a multiplier system of even (resp. odd) integral weight for $\Gamma$ by setting $\psi(\gamma):=\psi(d)$ for $\gamma=\left(\begin{array}{cc}a & b \\ c & d\end{array}\right)$.

Given a cusp $\mathfrak{a}$ let $\Gamma_{\mathfrak{a}}:=\{\gamma \in \Gamma: \gamma \mathfrak{a}=\mathfrak{a}\}$ denote its stabilizer in $\Gamma$, and let $\sigma_{\mathfrak{a}}$ denote the unique (up to translation by $\pm\left(\begin{array}{ll}1 & * \\ 0 & 1\end{array}\right)$ on the right) matrix in $\mathrm{SL}_{2}(\mathbb{R})$ satisfying $\sigma_{\mathfrak{a}} \infty=\mathfrak{a}$ and $\sigma_{\mathfrak{a}}^{-1} \Gamma_{\mathfrak{a}} \sigma_{\mathfrak{a}}=\Gamma_{\infty}$. Define $\alpha_{\nu, \mathfrak{a}} \in[0,1)$ by the condition

$$
\nu\left(\sigma_{\mathfrak{a}}\left(\begin{array}{ll}
1 & 1 \\
0 & 1
\end{array}\right) \sigma_{\mathfrak{a}}^{-1}\right)=e\left(-\alpha_{\nu, \mathfrak{a}}\right) .
$$

We say that $\mathfrak{a}$ is singular with respect to $\nu$ if $\nu$ is trivial on $\Gamma_{\mathfrak{a}}$, that is, if $\alpha_{\nu, \mathfrak{a}}=0$. For convenience we write $\alpha_{\nu}$ for $\alpha_{\nu, \mathfrak{a}}$ when $\mathfrak{a}=\infty$. Note that if $\alpha_{\nu}>0$ then

$$
\alpha_{\bar{\nu}}=1-\alpha_{\nu} .
$$

We are primarily interested in the multiplier system $\chi$ of weight $1 / 2$ on $\mathrm{SL}_{2}(\mathbb{Z}$ ) (and its conjugate $\bar{\chi}$ of weight $-1 / 2$ ) where

$$
\eta(\gamma \tau)=\chi(\gamma) \sqrt{c \tau+d} \eta(\tau), \quad \gamma=\left(\begin{array}{ll}
a & b \\
c & d
\end{array}\right) \in \mathrm{SL}_{2}(\mathbb{Z}),
$$

and $\eta$ is the Dedekind eta function defined in (1.12). From condition (ii) above we have $\chi(-I)=-i$. From the definition of $\eta(\tau)$ we have $\chi\left(\left(\begin{array}{lll}1 & 1 \\ 0 & 1\end{array}\right)\right)=e(1 / 24)$; it follows that

$$
\alpha_{\chi}=\frac{23}{24} \quad \text { and } \quad \alpha_{\bar{\chi}}=\frac{1}{24},
$$

so the unique cusp $\infty$ of $\mathrm{SL}_{2}(\mathbb{Z})$ is singular neither with respect to $\chi$ nor with respect to $\bar{\chi}$.

For $\gamma=\left(\begin{array}{ll}a & b \\ c & d\end{array}\right)$ with $c>0$ there are two useful formulas for $\chi$. Rademacher (see, for instance, (74.11), (74.12), and (71.21) of [R4]) showed that

$$
\chi(\gamma)=\sqrt{-i} e^{-\pi i s(d, c)} e\left(\frac{a+d}{24 c}\right),
$$


where $s(d, c)$ is the Dedekind sum

$$
s(d, c)=\sum_{r=1}^{c-1} \frac{r}{c}\left(\frac{d r}{c}-\left\lfloor\frac{d r}{c}\right\rfloor-\frac{1}{2}\right) .
$$

On the other hand, Petersson (see, for instance, $\S 4.1$ of [K] ) showed that

$$
\chi(\gamma)= \begin{cases}\left(\frac{d}{c}\right) e\left(\frac{1}{24}\left[(a+d) c-b d\left(c^{2}-1\right)-3 c\right]\right) & \text { if } c \text { is odd } \\ \left(\frac{c}{d}\right) e\left(\frac{1}{24}\left[(a+d) c-b d\left(c^{2}-1\right)+3 d-3-3 c d\right]\right) & \text { if } c \text { is even. }\end{cases}
$$

We will also use the multiplier system $\nu_{\theta}$ of weight $1 / 2$ on $\Gamma_{0}(4 N)$ defined by

$$
\theta(\gamma \tau)=\nu_{\theta}(\gamma) \sqrt{c \tau+d} \theta(\tau), \quad \gamma \in \Gamma_{0}(4 N),
$$

where

$$
\theta(\tau):=\sum_{n \in \mathbb{Z}} e\left(n^{2} \tau\right)
$$

Explicitly, we have

$$
\nu_{\theta}\left(\left(\begin{array}{ll}
a & b \\
c & d
\end{array}\right)\right)=\left(\frac{c}{d}\right) \varepsilon_{d}^{-1}
$$

where $(\vdots)$ is the extension of the Kronecker symbol given e.g. in [S] and

$$
\varepsilon_{d}=\left(\frac{-1}{d}\right)^{\frac{1}{2}}=\left\{\begin{array}{lll}
1 & \text { if } d \equiv 1 \quad(\bmod 4), \\
i & \text { if } d \equiv 3 \quad(\bmod 4)
\end{array}\right.
$$

Let $\Gamma_{0}(N, M)$ denote the subgroup of $\Gamma_{0}(N)$ consisting of matrices whose upper-right entry is divisible by $M$. Equation (2.9) shows that

$$
\chi(\gamma)=\left(\frac{c}{d}\right) e\left(\frac{d-1}{8}\right) \quad \text { for } \gamma=\left(\begin{array}{ll}
a & b \\
c & d
\end{array}\right) \in \Gamma_{0}(24,24) .
$$

This implies that

$$
\chi\left(\left(\begin{array}{cc}
a & 24 b \\
c / 24 & d
\end{array}\right)\right)=\left(\frac{12}{d}\right)\left(\frac{c}{d}\right) \varepsilon_{d}^{-1} \quad \text { for }\left(\begin{array}{ll}
a & b \\
c & d
\end{array}\right) \in \Gamma_{0}(576),
$$

which allows one to relate automorphic functions with multiplier $\chi$ on $\mathrm{SL}_{2}(\mathbb{Z})$ to those with multiplier $\left(\frac{12}{\bullet}\right) \nu_{\theta}$ on $\Gamma_{0}(576)$.

2.3. Maass forms. A function $f: \mathbb{H} \rightarrow \mathbb{C}$ is automorphic of weight $k$ and multiplier $\nu$ for $\Gamma=\Gamma_{0}(N)$ if

$$
\left.f\right|_{k} \gamma=\nu(\gamma) f \quad \text { for all } \gamma \in \Gamma
$$

Let $\mathcal{A}_{k}(N, \nu)$ denote the space of all such functions. A smooth, automorphic function which is also an eigenfunction of $\Delta_{k}$ and which satisfies the growth condition

$$
f(\tau) \ll y^{\sigma}+y^{1-\sigma} \quad \text { for some } \sigma \text {, for all } \tau \in \mathbb{H}
$$

is called a Maass form. We let $\mathcal{A}_{k}(N, \nu, r)$ denote the vector space of Maass forms with spectral parameter $r$. From the preceeding discussion, the raising (resp. lowering) operator maps $\mathcal{A}_{k}(N, \nu, r)$ into $\mathcal{A}_{k+2}(N, \nu, r)$ (resp. $\left.\mathcal{A}_{k-2}(N, \nu, r)\right)$. Also, complex conjugation $f \rightarrow \bar{f}$ gives a bijection $\mathcal{A}_{k}(N, \nu, r) \longleftrightarrow \mathcal{A}_{-k}(N, \bar{\nu}, r)$. 
If $f \in \mathcal{A}_{k}(n, \nu, r)$, then it satisfies $f(\tau+1)=e\left(-\alpha_{\nu}\right) f(\tau)$. For $n \in \mathbb{Z}$ define

$$
n_{\nu}:=n-\alpha_{\nu}
$$

Then $f$ has a Fourier expansion of the form

$$
f(\tau)=\sum_{n=-\infty}^{\infty} a(n, y) e\left(n_{\nu} x\right)
$$

By imposing condition (2.4) on the Fourier expansion we find that for $n_{\nu} \neq 0$, the function $a(n, y)$ satisfies

$$
\frac{a^{\prime \prime}(n, y)}{\left(4 \pi\left|n_{\nu}\right| y\right)^{2}}+\left(\frac{1 / 4+r^{2}}{\left(4 \pi\left|n_{\nu}\right| y\right)^{2}}+\frac{k \operatorname{sgn}\left(n_{\nu}\right)}{8 \pi\left|n_{\nu}\right| y}-\frac{1}{4}\right) a(n, y)=0 .
$$

The Whittaker functions $M_{\kappa, \mu}(y)$ and $W_{\kappa, \mu}(y)$ are the two linearly independent solutions to Whittaker's equation [DL, $§ 13.14]$

$$
W^{\prime \prime}+\left(\frac{1 / 4-\mu^{2}}{y^{2}}+\frac{\kappa}{y}-\frac{1}{4}\right) W=0 .
$$

As $y \rightarrow \infty$, the former solution grows exponentially, while the latter decays exponentially. Since $f$ satisfies the growth condition (2.12), we must have

$$
a(n, y)=a(n) W_{\frac{k}{2} \operatorname{sgn} n_{\nu}, i r}\left(4 \pi\left|n_{\nu}\right| y\right)
$$

for some constant $a(n)$. For $n_{\nu}=0$ we have

$$
a(n, y)=a(0) y^{\frac{1}{2}+i r}+a^{\prime}(0) y^{\frac{1}{2}-i r} .
$$

We call the numbers $a(n)$ (and $\left.a^{\prime}(0)\right)$ the Fourier coefficients of $f$. For $\operatorname{Re}(\mu-\kappa+1 / 2)>0$ we have the integral representation [DL, (13.14.3), (13.4.4)]

$$
W_{\kappa, \mu}(y)=\frac{e^{-y / 2} y^{\mu+1 / 2}}{\Gamma\left(\mu-\kappa+\frac{1}{2}\right)} \int_{0}^{\infty} e^{-y t} t^{\mu-\kappa-\frac{1}{2}}(1+t)^{\mu+\kappa-\frac{1}{2}} d t .
$$

When $\kappa=0$ we have [DL, (13.18.9)]

$$
W_{0, \mu}(y)=\frac{\sqrt{y}}{\sqrt{\pi}} K_{\mu}(y / 2)
$$

where $K_{\mu}$ is the $K$-Bessel function. For Maass forms of weight 0 , many authors normalize the Fourier coefficients in (2.14) so that $a(n)$ is the coefficient of $\sqrt{y} K_{i r}\left(2 \pi\left|n_{\nu}\right| y\right)$, which has the effect of multiplying $a(n)$ by $2\left|n_{\nu}\right|^{1 / 2}$.

By [DL, (13.15.26)], we have

$$
\begin{aligned}
y \frac{d}{d y} W_{\kappa, \mu}(y) & =\left(\frac{y}{2}-\kappa\right) W_{\kappa, \mu}(y)-W_{\kappa+1, \mu}(y) \\
& =\left(\kappa-\frac{y}{2}\right) W_{\kappa, \mu}(y)+\left(\kappa+\mu-\frac{1}{2}\right)\left(\kappa-\mu-\frac{1}{2}\right) W_{\kappa-1, \mu}(y) .
\end{aligned}
$$


This, together with [DL, (13.15.11)] shows that

$$
\begin{aligned}
& L_{k}\left(W_{\frac{k}{2} \operatorname{sgn}\left(n_{\nu}\right), i r}\left(4 \pi\left|n_{\nu}\right| y\right) e\left(n_{\nu} x\right)\right) \\
&=W_{\frac{k-2}{2} \operatorname{sgn}\left(n_{\nu}\right), i r}\left(4 \pi\left|n_{\nu}\right| y\right) e\left(n_{\nu} x\right) \times \begin{cases}-\left(r^{2}+\frac{(k-1)^{2}}{4}\right) & \text { if } n_{\nu}>0, \\
1 & \text { if } n_{\nu}<0,\end{cases}
\end{aligned}
$$

from which it follows that if $f_{L}:=L_{k} f$ has coefficients $a_{L}(n)$, then

$$
a_{L}(n)= \begin{cases}-\left(r^{2}+\frac{(k-1)^{2}}{4}\right) a(n) & \text { if } n_{\nu}>0, \\ a(n) & \text { if } n_{\nu}<0 .\end{cases}
$$

Similarly, for the coefficients $a_{R}(n)$ of $f_{R}:=R_{k} f$, we have

$$
a_{R}(n)= \begin{cases}-a(n) & \text { if } n_{\nu}>0 \\ \left(r^{2}+\frac{(k+1)^{2}}{4}\right) a(n) & \text { if } n_{\nu}<0\end{cases}
$$

From the integral representation (2.15) we find that $\overline{W_{\kappa, \mu}(y)}=W_{\kappa, \bar{\mu}}(y)$ when $y, \kappa \in \mathbb{R}$, so $W_{\kappa, \mu}(y)$ is real when $\mu \in \mathbb{R}$ or (by [DL, (13.14.31)]) when $\mu$ is purely imaginary. Suppose that $f$ has multiplier $\nu$ and that $\alpha_{\nu} \neq 0$. Then by (2.5) we have $-n_{\nu}=(1-n)_{\bar{\nu}}$, so the coefficients $a_{c}(n)$ of $f_{c}:=\bar{f}$ satisfy

$$
a_{c}(n)=\overline{a(1-n)} .
$$

2.4. The spectrum of $\Delta_{k}$. Let $\mathcal{L}_{k}(N, \nu)$ denote the $L^{2}$-space of automorphic functions with respect to the Petersson inner product

$$
\langle f, g\rangle:=\int_{\Gamma \backslash \mathbb{H}} f(\tau) \overline{g(\tau)} d \mu, \quad d \mu:=\frac{d x d y}{y^{2}} .
$$

Let $\mathcal{B}_{k}(N, \nu)$ denote the subspace of $\mathcal{L}_{k}(N, \nu)$ consisting of smooth functions $f$ such that $f$ and $\Delta_{k} f$ are bounded on $\mathbb{H}$. By (2.1), (2.2), and Green's formula, we have the relations (see also $\S 3$ of [Ro1])

$$
\begin{aligned}
\left\langle f,-\Delta_{k} g\right\rangle & =\left\langle R_{k} f, R_{k} g\right\rangle-\frac{k}{2}\left(1+\frac{k}{2}\right)\langle f, g\rangle \\
& =\left\langle L_{k} f, L_{k} g\right\rangle+\frac{k}{2}\left(1-\frac{k}{2}\right)\langle f, g\rangle
\end{aligned}
$$

for any $f, g \in \mathcal{B}_{k}(N, \nu)$. It follows that $\Delta_{k}$ is symmetric and that

$$
\left\langle f,-\Delta_{k} f\right\rangle \geq \lambda_{0}(k)\langle f, f\rangle, \quad \lambda_{0}(k):=\frac{|k|}{2}\left(1-\frac{|k|}{2}\right) .
$$

By Friedrichs' extension theorem (see e.g. [12, Appendix A.1]) the operator $\Delta_{k}$ has a unique self-adjoint extension to $\mathcal{L}_{k}(N, \nu)$ (denoted also by by $\Delta_{k}$ ).

From (2.20) we see that the spectrum of $\Delta_{k}$ is real and contained in $\left[\lambda_{0}(k), \infty\right)$. The holomorphic forms correspond to the bottom of the spectrum. To be precise, if $f_{0} \in \mathcal{L}_{k}(N, \nu)$ has eigenvalue $\lambda_{0}(k)$ then the equations above show that

$$
f_{0}(\tau)= \begin{cases}y^{\frac{k}{2}} F(\tau) & \text { if } k \geq 0 \\ y^{-\frac{k}{2}} \bar{F}(\tau) & \text { if } k<0\end{cases}
$$


where $F: \mathbb{H} \rightarrow \mathbb{C}$ is a holomorphic cusp form of weight $k$; i.e. $F$ vanishes at the cusps and satisfies

$$
F(\gamma \tau)=\nu(\gamma)(c \tau+d)^{k} F(\tau) \quad \text { for all } \gamma=\left(\begin{array}{ll}
a & b \\
c & d
\end{array}\right) \in \Gamma .
$$

In particular, $\lambda_{0}(k)$ is not an eigenvalue when the space of cusp forms is trivial. Note also that if $a_{0}(n)$ are the coefficients of $f_{0}$, then

$$
a_{0}(n)=0 \quad \text { when } \quad \operatorname{sgn}\left(n_{\nu}\right)=-\operatorname{sgn}(k) .
$$

The spectrum of $\Delta_{k}$ on $\mathcal{L}_{k}(N, \nu)$ consists of an absolutely continuous spectrum of multiplicity equal to the number of singular cusps, and a discrete spectrum of finite multiplicity. The Eisenstein series, of which there is one for each singular cusp $\mathfrak{a}$, give rise to the continuous spectrum, which is bounded below by $1 / 4$. When $(k, \nu)=(1 / 2, \chi)$ or $(-1 / 2, \bar{\chi})$ and $\Gamma=\mathrm{SL}_{2}(\mathbb{Z})$ there are no Eisenstein series since the only cusp is not singular.

Let $\mathcal{S}_{k}(N, \nu)$ denote the orthogonal complement in $\mathcal{L}_{k}(N, \nu)$ of the space generated by Eisenstein series. The spectrum of $\Delta_{k}$ on $\mathcal{S}_{k}(N, \nu)$ is countable and of finite multiplicity with no limit points except $\infty$. The exceptional eigenvalues are those which lie in $\left(\lambda_{0}(k), 1 / 4\right)$. Let $\lambda_{1}(N, \nu, k)$ denote the smallest eigenvalue larger than $\lambda_{0}(k)$ in the spectrum of $\Delta_{k}$ on $\mathcal{S}_{k}(N, \nu)$. Selberg's eigenvalue conjecture states that

$$
\lambda_{1}(N, \mathbf{1}, 0) \geq \frac{1}{4}
$$

i.e., there are no exceptional eigenvalues. Selberg [Se2] showed that

$$
\lambda_{1}(N, \mathbf{1}, 0) \geq \frac{3}{16} .
$$

The best progress toward this conjecture was made by Kim and Sarnak [Ki, Appendix 2], who proved that

$$
\lambda_{1}(N, \mathbf{1}, 0) \geq \frac{1}{4}-\left(\frac{7}{64}\right)^{2}=\frac{975}{4096},
$$

as a consequence of Langlands functoriality for the symmetric fourth power of an automorphic representation on $\mathrm{GL}_{2}$.

2.5. Maass cusp forms. The subspace $\mathcal{S}_{k}(N, \nu)$ consists of functions $f$ whose zeroth Fourier coefficient at each singular cusp vanishes. Eigenfunctions of $\Delta_{k}$ in $\mathcal{S}_{k}(N, \nu)$ are called Maass cusp forms. Let $\left\{f_{j}\right\}$ be an orthonormal basis of $\mathcal{S}_{k}(N, \nu)$, and for each $j$ let

$$
\lambda_{j}=\frac{1}{4}+r_{j}^{2}
$$

denote the Laplace eigenvalue and $\left\{a_{j}(n)\right\}$ the Fourier coefficients. Then we have the Fourier expansion

$$
f_{j}(\tau)=\sum_{n_{\nu} \neq 0} a_{j}(n) W_{\frac{k}{2} \operatorname{sgn} n_{\nu}, i_{j}}\left(4 \pi\left|n_{\nu}\right| y\right) e\left(n_{\nu} x\right)
$$

Suppose that $f$ has spectral parameter $r$. Then by (2.19) we have

$$
\left\|L_{k} f\right\|^{2}=\left(r^{2}+\frac{(k-1)^{2}}{4}\right)\|f\|^{2}
$$

Weyl's law describes the distribution of the spectral parameters $r_{j}$. Theorem 2.28 of [He] shows that

$$
\sum_{0 \leq r_{j} \leq T} 1-\frac{1}{4 \pi} \int_{-T}^{T} \frac{\varphi^{\prime}}{\varphi}\left(\frac{1}{2}+i t\right) d t=\frac{\operatorname{vol}(\Gamma \backslash \mathbb{H})}{4 \pi} T^{2}-\frac{K_{0}}{\pi} T \log T+O(T),
$$


where $\varphi(s)$ and $K_{0}$ are the determinant (see [He, p. 298]) and dimension (see [He, p. 281]), respectively, of the scattering matrix $\Phi(s)$ whose entries are given in terms of constant terms of Eisenstein series. In particular we have

$$
\sum_{0 \leq r_{j} \leq T} 1=\frac{1}{12} T^{2}+O(T) \quad \text { for }(k, \nu)=\left(\frac{1}{2}, \chi\right) .
$$

We will be working mostly in the space $\mathcal{S}_{\frac{1}{2}}(1, \chi)$. Throughout the paper we will denote an orthonormal basis for this space by $\left\{u_{j}\right\}$, and we will write the Fourier expansions as

$$
u_{j}(\tau)=\sum_{n \neq 0} \rho_{j}(n) W_{\frac{\operatorname{sgn} n}{4}, i r_{j}}(4 \pi|\widetilde{n}| y) e(\widetilde{n} x), \quad \widetilde{n}:=n-\frac{23}{24} .
$$

2.6. Hecke operators. We introduce Hecke operators on the spaces $\mathcal{S}_{0}(N, \mathbf{1})$ and $\mathcal{S}_{\frac{1}{2}}(1, \chi)$. For each prime $p \nmid N$ the Hecke operator $T_{p}$ acts on a Maass form $f \in \mathcal{A}_{0}(N, \mathbf{1})$ as

$$
T_{p} f(\tau)=p^{-\frac{1}{2}}\left(\sum_{j \bmod p} f\left(\frac{\tau+j}{p}\right)+f(p \tau)\right) .
$$

The $T_{p}$ commute with each other and with the Laplacian $\Delta_{0}$, so $T_{p}$ acts on each $\mathcal{S}_{0}(N, \mathbf{1}, r)$. The action of $T_{p}$ on the Fourier expansion of $f \in \mathcal{S}_{0}(N, \mathbf{1}, r)$ is given by

$$
T_{p} f(\tau)=\sum_{n \neq 0}\left(p^{\frac{1}{2}} a(p n)+p^{-\frac{1}{2}} a(n / p)\right) W_{0, i r}(4 \pi|n| y) e(n x) .
$$

Hecke operators on half-integral weight spaces have been defined for the theta multiplier (see e.g. [KS]). We define Hecke operators $T_{p^{2}}$ for $p \geq 5$ on $\mathcal{A}_{1 / 2}(1, \chi)$ directly by

$$
T_{p^{2}} f=\frac{1}{p}\left[\left.\sum_{b \bmod p^{2}} e\left(\frac{-b}{24}\right) f\right|_{\frac{1}{2}}\left(\begin{array}{cc}
\frac{1}{p} & \frac{b}{p} \\
0 & p
\end{array}\right)+\left.e\left(\frac{p-1}{8}\right) \sum_{h=1}^{p-1} e\left(\frac{-h p}{24}\right)\left(\frac{h}{p}\right) f\right|_{\frac{1}{2}}\left(\begin{array}{cc}
1 & \frac{h}{p} \\
0 & 1
\end{array}\right)+\left.f\right|_{\frac{1}{2}}\left(\begin{array}{ll}
p & 0 \\
0 & \frac{1}{p}
\end{array}\right)\right] .
$$

One can show that

$$
\left.\left(T_{p^{2}} f\right)\right|_{\frac{1}{2}}\left(\begin{array}{ll}
1 & 1 \\
0 & 1
\end{array}\right)=e\left(\frac{1}{24}\right) f \quad \text { and }\left.\quad\left(T_{p^{2}} f\right)\right|_{\frac{1}{2}}\left(\begin{array}{cc}
0 & -1 \\
1 & 0
\end{array}\right)=\sqrt{-i} f
$$

and that $T_{p^{2}}$ commutes with $\Delta_{\frac{1}{2}}$, so $T_{p^{2}}$ is an endomorphism of $\mathcal{S}_{\frac{1}{2}}(1, \chi, r)$. To describe the action of $T_{p^{2}}$ on Fourier expansions, it is convenient to write $f \in \mathcal{S}_{\frac{1}{2}}(1, \chi, r)$ in the form

$$
f(\tau)=\sum_{n \neq 0} a(n) W_{\frac{\mathrm{sgn}(n)}{4}, i r}\left(\frac{\pi|n| y}{6}\right) e\left(\frac{n x}{24}\right) .
$$

Then a standard computation gives

$$
T_{p^{2}} f(\tau)=\sum_{n \neq 0}\left(p a\left(p^{2} n\right)+p^{-\frac{1}{2}}\left(\frac{12 n}{p}\right) a(n)+p^{-1} a\left(n / p^{2}\right)\right) W_{\frac{\operatorname{sgn} n}{4}, i r}\left(\frac{\pi|n| y}{6}\right) e\left(\frac{n x}{24}\right) .
$$


2.7. Further operators. The reflection operator

$$
f(x+i y) \mapsto f(-x+i y)
$$

defines an involution on $\mathcal{S}_{0}(N, \mathbf{1})$ which commutes with $\Delta_{0}$ and the Hecke operators. We say that a Maass cusp form is even if it is fixed by reflection; such a form has Fourier expansion

$$
f(\tau)=\sum_{n \neq 0} a(n) W_{0, i r}(4 \pi|n| y) \cos (2 \pi n x) .
$$

We also have the operator

$$
f(\tau) \mapsto f(d \tau)
$$

which in general raises the level and changes the multiplier. We will only need this operator in the case $d=24$ on $\mathcal{S}_{1 / 2}(1, \chi)$, where (2.11) shows that

$$
f(\tau) \mapsto f(24 \tau): \mathcal{S}_{\frac{1}{2}}(1, \chi, r) \rightarrow \mathcal{S}_{\frac{1}{2}}\left(576,\left(\frac{12}{\bullet}\right) \nu_{\theta}, r\right)
$$

2.8. Generalized Kloosterman sums. Our main objects of study are the generalized Kloosterman sums given by

$$
S(m, n, c, \nu):=\sum_{\substack{0 \leq a, d<c \\
\gamma=\left(\begin{array}{ll}
a & b \\
c & d
\end{array}\right) \in \Gamma}} \bar{\nu}(\gamma) e\left(\frac{m_{\nu} a+n_{\nu} d}{c}\right)=\sum_{\substack{\gamma \in \Gamma_{\infty} \backslash \Gamma / \Gamma_{\infty} \\
\gamma=\left(\begin{array}{ll}
a & b \\
c & d
\end{array}\right)}} \bar{\nu}(\gamma) e\left(\frac{m_{\nu} a+n_{\nu} d}{c}\right)
$$

(to see that these are equal, one checks that each summand is invariant under the substitutions $\gamma \rightarrow\left(\begin{array}{ll}1 & 1 \\ 0 & 1\end{array}\right) \gamma$ and $\gamma \rightarrow \gamma\left(\begin{array}{ll}1 & 1 \\ 0 & 1\end{array}\right)$ using axiom (iii) for multiplier systems and (2.13) $)$. When $\Gamma=\mathrm{SL}_{2}(\mathbb{Z})$ and $\nu=\mathbf{1}$ we recover the ordinary Kloosterman sums

$$
S(m, n, c)=S(m, n, c, \mathbf{1})=\sum_{\substack{d \bmod c \\(d, c)=1}} e\left(\frac{m \bar{d}+n d}{c}\right),
$$

where $d \bar{d} \equiv 1(\bmod c)$. For the eta-multiplier, (2.7) gives

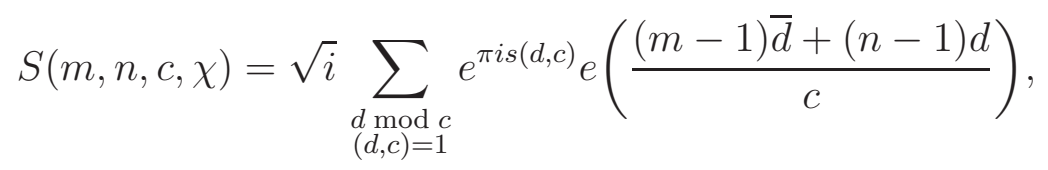

so the sums $A_{c}(n)$ appearing in Rademacher's formula are given by

$$
A_{c}(n)=\sqrt{-i} S(1,1-n, c, \chi) .
$$

We recall the bounds (1.4) and (1.11) from the introduction. A general Weil-type bound for $S(m, n, c, \chi)$ which holds for all $m, n$ follows from work of the second author, and is given in the following proposition.

Proposition 2.1. Let $m, n \in \mathbb{Z}$ and write $24 n-23=N$ and $24 m-23=v^{2} M$, with $M$ squarefree. For $c \geq 1$ we have

$$
|S(m, n, c, \chi)| \ll \tau((v, c)) \tau(c)(M N, c)^{\frac{1}{2}} \sqrt{c}
$$


Proof. We apply Theorem 3 of [A]. In the notation of that paper, we have $S(m, n, c, \chi)=$ $\sqrt{i} K(m-1, n-1 ; c)$. Estimating the right-hand side of [A, (1.20)] trivially, we find that

$$
|S(m, n, c, \chi)| \leq \frac{4}{\sqrt{3}} \tau((v, c)) c^{\frac{1}{2}} R(M N, 24 c),
$$

where

$$
R(y, \ell)=\#\left\{x \bmod \ell: x^{2} \equiv y \bmod \ell\right\} .
$$

The function $R(y, \ell)$ is multiplicative in $\ell$. If $p$ is prime and $p \nmid y$, then

$$
R\left(y, p^{e}\right) \leq \begin{cases}2 & \text { if } p \text { is odd } \\ 4 & \text { if } p=2\end{cases}
$$

If $y=p^{d} y^{\prime}$ with $p \nmid y^{\prime}$ then $R\left(y, p^{e}\right) \leq p^{d / 2} R\left(y^{\prime}, p^{e-d}\right)$. It follows that

$$
R(y, \ell) \leq 2 \cdot 2^{\omega(\ell)}(y, \ell)^{\frac{1}{2}}
$$

where $\omega(\ell)$ is the number of primes dividing $\ell$. By (2.27), (2.28), (2.29), and the fact that $2^{\omega(\ell)} \leq \tau(\ell)$, we obtain (2.26).

One useful consequence of Proposition 2.1] is the "trivial" estimate

$$
\sum_{c \leq X} \frac{|S(m, n, c, \chi)|}{c} \ll X^{\frac{1}{2}} \log X|m n|^{\epsilon},
$$

which follows from a standard argument involving the mean value bound for $\tau(c)$.

\section{A mean value estimate for Coefficients of MaAss Cusp forms}

In this section we prove a general version of Theorem 1.5 which applies to the Fourier coefficients of weight $\pm 1 / 2$ Maass cusp forms with multiplier $\nu$ for $\Gamma_{0}(N)$, where $\nu$ satisfies the following assumptions:

(1) There exists $\beta=\beta_{\nu} \in(1 / 2,1)$ such that

$$
\sum_{c>0} \frac{|S(n, n, c, \nu)|}{c^{1+\beta}} \ll_{\nu} n^{\epsilon} .
$$

(2) None of the cusps of $\Gamma_{0}(N)$ is singular for $\nu$.

By (2.30) the multipliers $\chi$ and $\bar{\chi}$ on $\mathrm{SL}_{2}(\mathbb{Z})$ satisfy these assumptions with $\beta=1 / 2+\epsilon$.

Fix an orthonormal basis of cusp forms $\left\{f_{j}\right\}$ for $\mathcal{S}_{k}\left(\Gamma_{0}(N), \nu\right)$. For each $j$, let $a_{j}(n)$ denote the $n$-th Fourier coefficient of $f_{j}$ and let $r_{j}$ denote the spectral parameter.

Theorem 3.1. Suppose that $k= \pm 1 / 2$ and that $\nu$ satisfies conditions (1) and (2) above. Then for all $x \geq 2$ and $n \geq 1$ we have

$$
n_{\nu} \sum_{0<r_{j} \leq x} \frac{\left|a_{j}(n)\right|^{2}}{\operatorname{ch} \pi r_{j}}= \begin{cases}\frac{x^{\frac{3}{2}}}{3 \pi^{2}}+O_{\nu}\left(x^{\frac{1}{2}} \log x+n^{\beta+\epsilon}\right) & \text { if } k=1 / 2, \\ \frac{x^{\frac{5}{2}}}{5 \pi^{2}}+O_{\nu}\left(x^{\frac{3}{2}} \log x+n^{\beta+\epsilon} x^{\frac{1}{2}}\right) & \text { if } k=-1 / 2 .\end{cases}
$$

The $n>0$ case of Theorem 1.5 follows from a direct application of Theorem 3.1 when $(k, \nu)=(1 / 2, \chi)$. For $n<0$, we apply Theorem 3.1 to the case $(k, \nu)=(-1 / 2, \bar{\chi})$ and use the relation (2.17). 
3.1. An auxiliary version of the Kuznetsov trace formula. We begin with an auxiliary version of Kuznetsov's formula $([\mathrm{Ku}, \S 5])$ which is Lemma 3 of [P2] with $m=n$. By assumption (2) there are no Eisenstein series for the multiplier system $\nu$. While Proskurin assumes that $k>0$ throughout his paper, this lemma is still valid for $k<0$ by the same proof. We include the term $\Gamma(2 \sigma-1)$ which is omitted on the right-hand side of [P2, Lemma 3].

Lemma 3.2. Suppose that $k= \pm 1 / 2$ and that $\nu$ satisfies conditions (1) and (2) above. For $n>0, t \in \mathbb{R}$, and $\sigma>1$ we have

$$
\begin{aligned}
& -\frac{1}{i^{k+1}} \sum_{c>0} \frac{S(n, n, c, \nu)}{c^{2 \sigma}} \int_{L} K_{i t}\left(\frac{4 \pi n_{\nu}}{c} q\right)\left(q+\frac{1}{q}\right)^{2 \sigma-2} q^{k-1} d q \\
& \quad=\frac{\Gamma(2 \sigma-1)}{4\left(2 \pi n_{\nu}\right)^{2 \sigma-1}}-\frac{2^{1-2 \sigma} \pi^{2-2 \sigma} n_{\nu}^{2-2 \sigma}}{\Gamma\left(\sigma-\frac{k}{2}+\frac{i t}{2}\right) \Gamma\left(\sigma-\frac{k}{2}-\frac{i t}{2}\right)} \sum_{r_{j}}\left|a_{j}(n)\right|^{2} \Lambda\left(\sigma+\frac{i t}{2}, \sigma-\frac{i t}{2}, r_{j}\right),
\end{aligned}
$$

where $L$ is the contour $|q|=1, \operatorname{Re}(q)>0$, from $-i$ to $i$ and

$$
\Lambda\left(s_{1}, s_{2}, r\right)=\Gamma\left(s_{1}-\frac{1}{2}-i r\right) \Gamma\left(s_{1}-\frac{1}{2}+i r\right) \Gamma\left(s_{2}-\frac{1}{2}-i r\right) \Gamma\left(s_{2}-\frac{1}{2}+i r\right) .
$$

We justify substituting $\sigma=1$ in this lemma. Suppose that $t \in \mathbb{R}$ and write $a=4 \pi n_{\nu} / c$ and $q=e^{i \theta}$ with $-\pi / 2<\theta<\pi / 2$. By [DL, (10.27.4), (10.25.2), and (5.6.7)] we have, for $c$ sufficiently large,

$$
K_{i t}\left(a e^{i \theta}\right) \ll e^{-\frac{\pi|t|}{2}}\left(e^{\theta t}+e^{-\theta t}\right) .
$$

So $K_{i t}(a q) \ll 1$ on $L$ and the integral over $L$ is bounded uniformly in $a$. By this and the assumption (3.1), the left-hand side of (3.3) is absolutely uniformly convergent for $\sigma \in[1,2]$. In this range we have

$$
\left|\Gamma\left(\sigma-\frac{1}{2}+i y\right)\right|=\frac{\left|\Gamma\left(\sigma+\frac{1}{2}+i y\right)\right|}{\left|\sigma-\frac{1}{2}+i y\right|} \leq 2\left|\Gamma\left(\sigma+\frac{1}{2}+i y\right)\right| .
$$

Using this together with the fact that $\Lambda\left(\sigma+\frac{i t}{2}, \sigma-\frac{i t}{2}, r_{j}\right)$ is positive, we see that the convergence of the right hand side of (3.3) for $\sigma>1$ implies the uniform convergence of the right hand side for $\sigma \in[1,2]$. We may therefore take $\sigma=1$.

To evaluate the integral on the left-hand side of (3.3) we require the following lemma.

Lemma 3.3. Suppose that $a>0$. Then

$$
\begin{aligned}
\int_{L} K_{i t}(a q) q^{k-1} d q \\
=i \sqrt{2} \times \begin{cases}\frac{1}{\sqrt{a}} \int_{0}^{a} u^{-\frac{1}{2}} \int_{0}^{\infty} \cos (t \xi)(\cos (u \operatorname{ch} \xi)-\sin (u \operatorname{ch} \xi)) d \xi d u & \text { if } k=\frac{1}{2}, \\
\sqrt{a} \int_{a}^{\infty} u^{-\frac{3}{2}} \int_{0}^{\infty} \cos (t \xi)(\cos (u \operatorname{ch} \xi)+\sin (u \operatorname{ch} \xi)) d \xi d u & \text { if } k=-\frac{1}{2} .\end{cases}
\end{aligned}
$$

Proof. The case $k=1 / 2$ follows from equation (44) in [P2] (correcting a typo in the upper limit of integration) and the integral representation [DL, (10.9.8)].

For $k=-1 / 2$, we write

$$
\int_{L} K_{i t}(a q) q^{k-1} d q=-\left(\int_{-i R}^{-i}+\int_{i}^{i R}+\int_{L_{R}}\right) K_{i t}(a q) q^{k-1} d q
$$


where the first two integrals are along the imaginary axis, and $L_{R}$ is the semicircle $|q|=R$, $\operatorname{Re}(q)>0$, traversed clockwise. By [DL, (10.40.10)], the integral over $L_{R}$ approaches zero as $R \rightarrow \infty$. For the first and second integrals we change variables to $q=-i u / a$ and $q=i u / a$, respectively, to obtain

$$
\int_{L} K_{i t}(a q) q^{k-1} d q=-\sqrt{a} \int_{a}^{\infty}\left(\sqrt{-i} K_{i t}(i u)-\sqrt{i} K_{i t}(-i u)\right) \frac{d u}{u^{3 / 2}} .
$$

The lemma follows from writing $K_{i t}( \pm i u)$ as a linear combination of $J_{i t}(u)$ and $J_{-i t}(u)$ using [DL, (10.27.9), (10.27.3)] and applying the integral representation [DL, (10.9.8)].

Starting with (3.3) with $\sigma=1$ and using the fact that

$$
\Lambda(1+i t, 1-i t, r)=\frac{\pi^{2}}{\operatorname{ch}(\pi r+\pi t) \operatorname{ch}(\pi r-\pi t)},
$$

we replace $t$ by $2 t$, multiply by

$$
\frac{4 n_{\nu}}{\pi^{2}} g(t), \quad \text { where } g(t):=\left|\Gamma\left(1-\frac{k}{2}+i t\right)\right|^{2} \operatorname{sh} \pi t,
$$

and integrate on $t$ from 0 to $x$. Applying Lemma 3.3 to the result, we obtain

$$
n_{\nu} \sum_{r_{j}} \frac{\left|a_{j}(n)\right|^{2}}{\operatorname{ch} \pi r_{j}} h_{x}\left(r_{j}\right)=\frac{1}{2 \pi^{3}} \int_{0}^{x} g(t) d t+\frac{\left(4 n_{\nu}\right)^{1-k} \sqrt{2}}{i^{k} \pi^{2+k}} \sum_{c>0} \frac{S(n, n, c, \nu)}{c^{2-k}} I\left(x, \frac{4 \pi n_{\nu}}{c}\right),
$$

where

$$
h_{x}(r):=\int_{0}^{x} \frac{2 \operatorname{sh} \pi t \operatorname{ch} \pi r}{\operatorname{ch}(\pi r+\pi t) \operatorname{ch}(\pi r-\pi t)} d t
$$

and

$$
I(x, a):= \begin{cases}\int_{0}^{x} g(t) \int_{0}^{a} u^{-\frac{1}{2}} \int_{0}^{\infty} \cos (2 t \xi)(\cos (u \operatorname{ch} \xi)-\sin (u \operatorname{ch} \xi)) d \xi d u d t & \text { if } k=\frac{1}{2}, \\ \int_{0}^{x} g(t) \int_{a}^{\infty} u^{-\frac{3}{2}} \int_{0}^{\infty} \cos (2 t \xi)(\cos (u \operatorname{ch} \xi)+\sin (u \operatorname{ch} \xi)) d \xi d u d t & \text { if } k=-\frac{1}{2} .\end{cases}
$$

3.2. The function $g(t)$ and the main term. A computation involving [DL, (5.11.3), (5.11.11)] shows that for fixed $x$ and $y \geq 1$ we have

$$
\Gamma(x+i y) \Gamma(x-i y)=2 \pi y^{2 x-1} e^{-\pi y}\left(1+O\left(\frac{1}{y}\right)\right),
$$

from which it follows that

$$
g(t)=\pi t^{1-k}+O\left(t^{-k}\right) \quad \text { as } t \rightarrow \infty .
$$

We have

$$
g(t)=\pi \Gamma\left(1-\frac{k}{2}\right)^{2} t+O\left(t^{2}\right) \quad \text { as } t \rightarrow 0 .
$$

This gives the main term

$$
\frac{1}{2 \pi^{3}} \int_{0}^{x} g(t) d t= \begin{cases}\frac{x^{\frac{3}{2}}}{3 \pi^{2}}+O\left(x^{\frac{1}{2}}\right) & \text { if } k=\frac{1}{2} \\ \frac{x^{\frac{5}{2}}}{5 \pi^{2}}+O\left(x^{\frac{3}{2}}\right) & \text { if } k=-\frac{1}{2}\end{cases}
$$


3.3. Preparing to estimate the error term. The error term in (3.2) is obtained by uniformly estimating the integral $I(x, a)$ in the ranges $a \leq 1$ and $a \geq 1$. The analysis is similar in spirit to [Ku , Section 5], but the nature of the function $g(t)$ introduces substantial difficulties. In this section we collect a number of facts which we will require for these estimates.

Given an interval $[a, b]$, let var $f$ denote the total variation of $f$ on $[a, b]$ which, for $f$ differentiable, is given by

$$
\operatorname{var} f=\int_{a}^{b}\left|f^{\prime}(x)\right| d x .
$$

We begin by collecting some facts about the functions $g^{\prime}(t),(g(t) / t)^{\prime}$, and $t(g(t) / t)^{\prime}$.

Lemma 3.4. Let $k= \pm 1 / 2$. If $x \geq 1$ then on the interval $[0, x]$ we have

$$
\begin{aligned}
\operatorname{var}(g(t) / t)^{\prime} & \ll 1+x^{-1-k}, \\
\operatorname{var} t(g(t) / t)^{\prime} & \ll 1+x^{-k} .
\end{aligned}
$$

For $t>0$ we have

$$
g^{\prime}(t)>0, \quad \operatorname{sgn}(g(t) / t)^{\prime}=-\operatorname{sgn} k .
$$

Proof. Let $\sigma=1-k / 2$. Taking the logarithmic derivative of $g(t) / t$, we find that

$$
\left(\frac{g(t)}{t}\right)^{\prime}=g(t) h(t)
$$

where

We have

$$
h(t):=\frac{1}{t}\left[i(\psi(\sigma+i t)-\psi(\sigma-i t))-\frac{1}{t}+\pi \operatorname{coth} \pi t\right], \quad \psi:=\frac{\Gamma^{\prime}}{\Gamma} .
$$

and

$$
g^{\prime}(t)=g(t)[i(\psi(\sigma+i t)-\psi(\sigma-i t))+\pi \operatorname{coth} \pi t]
$$

$$
h^{\prime}(t)=-\frac{h(t)}{t}-\frac{1}{t}\left[\psi^{\prime}(\sigma+i t)+\psi^{\prime}(\sigma-i t)-\frac{1}{t^{2}}+\pi^{2}(\operatorname{csch} \pi t)^{2}\right] .
$$

At $t=0$ we have the Taylor series

$$
\begin{aligned}
& g(t)=\pi \Gamma(\sigma)^{2} t+\pi \Gamma(\sigma)^{2}\left(\frac{\pi^{2}}{6}-\psi^{\prime}(\sigma)\right) t^{3}+\ldots, \\
& h(t)=\frac{\pi^{2}}{3}-2 \psi^{\prime}(\sigma)+\left(\frac{1}{3} \psi^{\prime \prime \prime}(\sigma)-\frac{\pi^{4}}{45}\right) t^{2}+\ldots
\end{aligned}
$$

Thus we have the estimates

$$
g(t) \ll t, \quad g^{\prime}(t) \ll 1, \quad h(t) \ll 1, \quad \text { and } \quad h^{\prime}(t) \ll t \quad \text { as } t \rightarrow 0 .
$$

For large $t$ we use [DL, (5.11.2) and (5.15.8)] to obtain the asymptotic expansions

$$
\begin{aligned}
i(\psi(\sigma+i t)-\psi(\sigma-i t)) & =-2 \arctan \left(\frac{t}{\sigma}\right)-\frac{t}{\sigma^{2}+t^{2}}+O\left(\frac{1}{t^{2}}\right), \\
\psi^{\prime}(\sigma+i t)+\psi^{\prime}(\sigma-i t) & =\frac{2 \sigma}{\sigma^{2}+t^{2}}+\frac{\sigma^{2}-t^{2}}{\left(\sigma^{2}+t^{2}\right)^{2}}+O\left(\frac{1}{t^{3}}\right) .
\end{aligned}
$$

By (3.8), (3.16), and (3.17) we have the estimates

$$
g(t) \ll t^{1-k}, \quad g^{\prime}(t) \ll t^{-k}, \quad h(t) \ll \frac{1}{t^{2}}, \quad \text { and } \quad h^{\prime}(t) \ll \frac{1}{t^{3}} \quad \text { as } t \rightarrow \infty .
$$


From (3.18) and (3.11) it follows that

$$
\operatorname{var}(g(t) / t)^{\prime}=\int_{0}^{x}\left|g(t) h^{\prime}(t)+g^{\prime}(t) h(t)\right| d t \ll 1+x^{-1-k}
$$

and

$$
\operatorname{var} t(g(t) / t)^{\prime}=\int_{0}^{x}\left|t g(t) h^{\prime}(t)+t g^{\prime}(t) h(t)+g(t) h(t)\right| d t \ll 1+x^{-k} .
$$

Using [DL, (4.36.3), (5.7.6)] we have

$$
h(t)=2 \sum_{n=1}^{\infty}\left(\frac{1}{t^{2}+n^{2}}-\frac{1}{t^{2}+\left(n-\frac{k}{2}\right)^{2}}\right) .
$$

The second claim in (3.14) follows from (3.15). The first is simpler.

Suppose that $f \geq 0$ and $\phi$ are of bounded variation on $[a, b]$ with no common points of discontinuity. By Corollary 2 of $[\mathrm{Kn}]$, there exists $[\alpha, \beta] \subseteq[a, b]$ such that

$$
\int_{a}^{b} f d \phi=(\inf f+\operatorname{var} f) \int_{\alpha}^{\beta} d \phi
$$

where the integrals are of Riemann-Stieltjes type. We obtain the following by taking $\phi(x)=$ $\int_{0}^{x} G(t) d t$.

Lemma 3.5. Suppose that $F \geq 0$ and $G$ are of bounded variation on $[a, b]$ and that $G$ is continuous. Then

$$
\left|\int_{a}^{b} F(x) G(x) d x\right| \leq(\inf F+\operatorname{var} F) \sup _{[\alpha, \beta] \subseteq[a, b]}\left|\int_{\alpha}^{\beta} G(x) d x\right| .
$$

We will frequently make use of the following well-known estimates for oscillatory integrals (see, for instance, [T, Chapter IV]).

Lemma 3.6 (First derivative estimate). Suppose that $F$ and $G$ are real-valued functions on $[a, b]$ with $F$ differentiable, such that $G(x) / F^{\prime}(x)$ is monotonic. If $\left|F^{\prime}(x) / G(x)\right| \geq m>0$ then

$$
\int_{a}^{b} G(x) e(F(x)) d x \ll \frac{1}{m} .
$$

Lemma 3.7 (Second derivative estimate). Suppose that $F$ and $G$ are real-valued functions on $[a, b]$ with $F$ twice differentiable, such that $G(x) / F^{\prime}(x)$ is monotonic. If $|G(x)| \leq M$ and $\left|F^{\prime \prime}(x)\right| \geq m>0$ then

$$
\int_{a}^{b} G(x) e(F(x)) d x \ll \frac{M}{\sqrt{m}} .
$$

3.4. The first error estimate. We estimate the error arising from $I(x, a)$ (recall (3.7)) when $k=1 / 2$.

Proposition 3.8. Suppose that $k=1 / 2$. For $a>0$ we have

$$
I(x, a) \ll \begin{cases}\sqrt{a}(\log (1 / a)+1) & \text { if } a \leq 1 \\ 1 & \text { if } a \geq 1 .\end{cases}
$$


Proof. We write $I(x, a)$ as a difference of integrals, one involving $\cos (u \operatorname{ch} \xi)$, the other involving $\sin (u \operatorname{ch} \xi)$. We will estimate the first integral, the second being similar. We have

$$
\int_{0}^{\infty} \cos (2 t \xi) \cos (u \operatorname{ch} \xi) d \xi=\int_{0}^{T} \cos (2 t \xi) \cos (u \operatorname{ch} \xi) d \xi+R_{T}(u, t),
$$

where, by the second derivatve estimate,

$$
R_{T}(u, t) \ll u^{-\frac{1}{2}} e^{-\frac{T}{2}} .
$$

For $0<\varepsilon<a$ we have, using (3.8),

$$
\int_{0}^{x} g(t) \int_{\varepsilon}^{a} u^{-\frac{1}{2}} R_{T}(u, t) d u d t \ll e^{-\frac{T}{2}} x^{\frac{3}{2}}(|\log a|+|\log \varepsilon|) \rightarrow 0 \quad \text { as } T \rightarrow \infty .
$$

So our goal is to estimate

$$
\lim _{\varepsilon \rightarrow 0^{+}} \lim _{T \rightarrow \infty} I(x, \varepsilon, a, T)
$$

where

$$
I(x, \varepsilon, a, T)=\int_{0}^{x} g(t) \int_{\varepsilon}^{a} u^{-\frac{1}{2}} \int_{0}^{T} \cos (2 t \xi) \cos (u \operatorname{ch} \xi) d \xi d u d t .
$$

Integrating the innermost integral by parts, we find that

$$
\begin{aligned}
I(x, \varepsilon, a, T)=\frac{1}{2} \int_{0}^{x} \frac{g(t)}{t} \sin (2 t T) d t \int_{\varepsilon}^{a} u^{-\frac{1}{2}} \cos (u \operatorname{ch} T) d u \\
+\frac{1}{2} \int_{0}^{T} \operatorname{sh} \xi \int_{0}^{x} \frac{g(t)}{t} \sin (2 t \xi) d t \int_{\varepsilon}^{a} \sqrt{u} \sin (u \operatorname{ch} \xi) d u d \xi .
\end{aligned}
$$

The first derivative estimate (recalling (3.9) and (3.14)) gives

$$
\int_{0}^{x} \frac{g(t)}{t} \sin (2 t T) d t \ll \frac{1}{T} \quad \text { and } \quad \int_{\varepsilon}^{a} u^{-\frac{1}{2}} \cos (u \operatorname{ch} T) d u \ll \frac{1}{\sqrt{\varepsilon} \operatorname{ch} T},
$$

so the first term on the right-hand side of (3.19) approaches zero as $T \rightarrow \infty$.

Turning to the second term, set

$$
G_{x}(\xi):=\int_{0}^{x} \frac{g(t)}{t} \sin (2 t \xi) d t
$$

Integrating by parts gives

$$
G_{x}(\xi)=\frac{\pi \Gamma\left(\frac{3}{4}\right)^{2}}{2 \xi}-\frac{g(x)}{x} \frac{\cos (2 x \xi)}{2 \xi}+\frac{1}{2 \xi} \int_{0}^{x}\left(\frac{g(t)}{t}\right)^{\prime} \cos (2 t \xi) d t .
$$

Applying Lemma 3.5 and (3.12) to the integral in (3.20) we find that

$$
\frac{1}{\xi} \int_{0}^{x}\left(\frac{g(t)}{t}\right)^{\prime} \cos (2 t \xi) d t \ll \frac{1}{\xi} \sup _{0 \leq \alpha<\beta \leq x}\left|\int_{\alpha}^{\beta} \cos (2 t \xi) d t\right| \ll \frac{1}{\xi^{2}} .
$$

On the other hand, (3.14) gives

$$
\left|\frac{1}{\xi} \int_{0}^{x}\left(\frac{g(t)}{t}\right)^{\prime} \cos (2 t \xi) d t\right| \leq \frac{1}{\xi} \int_{0}^{x}-\left(\frac{g(t)}{t}\right)^{\prime} d t \ll \frac{1}{\xi} .
$$

It follows that

$$
G_{x}(\xi)=\frac{\pi \Gamma\left(\frac{3}{4}\right)^{2}}{2 \xi}-\frac{g(x)}{x} \frac{\cos (2 x \xi)}{2 \xi}+O\left(\min \left(\xi^{-1}, \xi^{-2}\right)\right)
$$


The integral

$$
\int_{\varepsilon}^{a} \sqrt{u} \sin (u \operatorname{ch} \xi) d u
$$

evaluates to $H(a, \xi)-H(\varepsilon, \xi)$, where

$$
H(u, \xi):=-\frac{\sqrt{u} \cos (u \operatorname{ch} \xi)}{\operatorname{ch} \xi}+\frac{\sqrt{\pi / 2}}{(\operatorname{ch} \xi)^{\frac{3}{2}}} C\left(\sqrt{\frac{2 u \operatorname{ch} \xi}{\pi}}\right),
$$

and $C(x)$ denotes the Fresnel integral

$$
C(x):=\int_{0}^{x} \cos \left(\frac{\pi}{2} t^{2}\right) d t .
$$

For $u>0$, write

$$
J(x, u):=\int_{0}^{\infty} \operatorname{sh} \xi G_{x}(\xi) H(u, \xi) d \xi
$$

To show that $J(x, u)$ converges, suppose that $B \geq A \geq T$. By (3.22), (3.23), and the bound $C(x) \leq 1$ we have

$$
\begin{aligned}
& \int_{A}^{B} \operatorname{sh} \xi G_{x}(\xi) H(u, \xi) d \xi \\
& \quad=-\sqrt{u} \int_{A}^{B} \operatorname{th} \xi \cos (u \operatorname{ch} \xi)\left(\frac{\pi \Gamma\left(\frac{3}{4}\right)^{2}}{2 \xi}-\frac{g(x)}{x} \frac{\cos (2 x \xi)}{2 \xi}+O\left(\frac{1}{\xi^{2}}\right)\right) d \xi+O\left(\int_{A}^{B} \frac{\operatorname{sh} \xi}{\xi(\operatorname{ch} \xi)^{\frac{3}{2}}} d \xi\right) \\
& \quad \ll u^{-\frac{1}{2}} e^{-A / 2}+u^{\frac{1}{2}} A^{-1}+e^{-A / 2}
\end{aligned}
$$

where we have used the first derivative estimate in the last inequality. We have

$$
\lim _{\varepsilon \rightarrow 0^{+}} \lim _{T \rightarrow \infty} I(x, \varepsilon, a, T)=\frac{1}{2} J(x, a)-\frac{1}{2} \lim _{\varepsilon \rightarrow 0^{+}} J(x, \varepsilon),
$$

so it remains only to estimate $J(x, u)$ in the ranges $u \leq 1$ and $u \geq 1$.

First, suppose that $u \leq 1$. We estimate each of the six terms obtained by multiplying (3.22) and (3.23) in (3.24). Starting with the terms involving $\cos (u \operatorname{ch} \xi)$, we have

$$
\begin{aligned}
\sqrt{u} \int_{0}^{\infty} \frac{\operatorname{th} \xi}{\xi} \cos (u \operatorname{ch} \xi) d \xi & =\sqrt{u}\left(\int_{0}^{\log \frac{1}{u}}+\int_{\log \frac{1}{u}}^{\infty}\right) \frac{\operatorname{th} \xi}{\xi} \cos (u \operatorname{ch} \xi) d \xi \\
& \ll \sqrt{u}(\log (1 / u)+1)
\end{aligned}
$$

by applying the second derivative estimate to the second integral. By the same method,

$$
\sqrt{u} \frac{g(x)}{x} \int_{0}^{\infty} \frac{\operatorname{th} \xi}{\xi} \cos (2 x \xi) \cos (u \operatorname{ch} \xi) d \xi \ll \sqrt{u}(\log (1 / u)+1),
$$

since $g(x) / x \ll 1$. For the third term we have

$$
\sqrt{u} \int_{0}^{\infty} \operatorname{th} \xi \cos (u \operatorname{ch} \xi) \min \left(\xi^{-1}, \xi^{-2}\right) d \xi \ll \sqrt{u} \int_{0}^{1} \frac{\operatorname{th} \xi}{\xi} d \xi+\sqrt{u} \int_{1}^{\infty} \frac{\operatorname{th} \xi}{\xi^{2}} d \xi \ll \sqrt{u} .
$$


For the terms involving the Fresnel integral, we use the trivial estimate $C(x) \leq \min (x, 1)$. For the first of these terms we have

$$
\int_{0}^{\infty} \frac{\operatorname{sh} \xi}{\xi(\operatorname{ch} \xi)^{\frac{3}{2}}} C\left(\sqrt{\frac{2 u \operatorname{ch} \xi}{\pi}}\right) d \xi \ll \sqrt{u} \int_{0}^{\log \frac{1}{u}} \frac{\operatorname{th} \xi}{\xi} d \xi+\int_{\log \frac{1}{u}}^{\infty} \frac{d \xi}{\sqrt{\operatorname{ch} \xi}} \ll \sqrt{u}(\log (1 / u)+1) .
$$

By the same method, the remaining two terms are $\ll \sqrt{u}(\log (1 / u)+1)$. It follows that

$$
J(x, u) \ll \sqrt{u}(\log (1 / u)+1) \quad \text { for } u \leq 1 .
$$

For $u \geq 1$ we show that $J(x, u) \ll 1$. Write

$$
J(x, u)=J_{1}(x, u)+J_{2}(x, u):=\left(\int_{0}^{1 / \sqrt{u}}+\int_{1 / \sqrt{u}}^{\infty}\right) \operatorname{sh} \xi G_{x}(\xi) H(u, \xi) d \xi .
$$

Since $G_{x}(\xi) \ll \xi^{-1}$ and $H(u, \xi) \ll \sqrt{u} / \operatorname{ch} \xi$, we have

$$
J_{1}(x, u) \ll \sqrt{u} \int_{0}^{1 / \sqrt{u}} \frac{\operatorname{th} \xi}{\xi} d \xi \ll 1 .
$$

We break $J_{2}(x, u)$ into six terms, using (3.20) in place of (3.22). We start with the terms involving $\pi \Gamma(3 / 4)^{2} / 2 \xi$. By the first derivative estimate we have

$$
\sqrt{u} \int_{1 / \sqrt{u}}^{\infty} \frac{\operatorname{th} \xi}{\xi} \cos (u \operatorname{ch} \xi) d \xi \ll 1 .
$$

Estimating the Fresnel integral trivially, we have

$$
\int_{1 / \sqrt{u}}^{\infty} \frac{\operatorname{sh} \xi}{\xi(\operatorname{ch} \xi)^{\frac{3}{2}}} C\left(\sqrt{\frac{2 u \operatorname{ch} \xi}{\pi}}\right) d \xi \ll \int_{0}^{\infty} \frac{d \xi}{\sqrt{\operatorname{ch} \xi}} \ll 1 .
$$

The two terms involving $\cos (2 x \xi) / \xi$ are treated similarly, and are $\ll 1$. The term involving the integral in (3.20) and the Fresnel integral can be estimated trivially using (3.21); it is also $\ll 1$.

The final term,

$$
\sqrt{u} \int_{1 / \sqrt{u}}^{\infty} \frac{\operatorname{sh} \xi \cos (u \operatorname{ch} \xi)}{\xi \operatorname{ch} \xi} \int_{0}^{x}\left(\frac{g(t)}{t}\right)^{\prime} \cos (2 t \xi) d t d \xi
$$

is more delicate. We write the integral as

$$
\sqrt{u} \int_{1 / \sqrt{u}}^{\infty}\left\{\frac{1}{\xi \operatorname{ch} \xi} \int_{0}^{x}\left(\frac{g(t)}{t}\right)^{\prime} \cos (2 t \xi) d t\right\} \cdot\{\operatorname{sh} \xi \cos (u \operatorname{ch} \xi) d \xi\}=\sqrt{u} \int_{1 / \sqrt{u}}^{\infty} U d V
$$

and integrate by parts. By (3.21) we have

$$
\left.\sqrt{u} U V\right|_{1 / \sqrt{u}} ^{\infty} \ll \sqrt{u} \cdot \frac{\sqrt{u}}{\operatorname{ch}(1 / \sqrt{u})} \cdot \frac{1}{u} \ll 1 .
$$

Write $U^{\prime}=R+S$, where

$$
R=\left(\frac{1}{\xi \operatorname{ch} \xi}\right)^{\prime} \int_{0}^{x}\left(\frac{g(t)}{t}\right)^{\prime} \cos (2 t \xi) d t, \quad S=\frac{2}{\xi \operatorname{ch} \xi} \int_{0}^{x} t\left(\frac{g(t)}{t}\right)^{\prime} \sin (2 t \xi) d t .
$$


By (3.21) we have

$$
R \ll \frac{1}{\xi \operatorname{ch} \xi}\left(\frac{1}{\xi}+\operatorname{th} \xi\right) \ll \begin{cases}\left(\xi^{2} \operatorname{ch} \xi\right)^{-1} & \text { if } \xi \leq 1 \\ (\xi \operatorname{ch} \xi)^{-1} & \text { if } \xi \geq 1\end{cases}
$$

Applying Lemma 3.5 and the estimate (3.13) we find that

$$
S \ll \frac{1}{\xi^{2} \operatorname{ch} \xi} \text {. }
$$

Thus we have

$$
\sqrt{u} \int_{1 / \sqrt{u}}^{\infty} V d U \ll \frac{1}{\sqrt{u}} \int_{1 / \sqrt{u}}^{1} \frac{d \xi}{\xi^{2}}+\frac{1}{\sqrt{u}} \int_{1}^{\infty} \frac{d \xi}{\xi \operatorname{ch} \xi} \ll 1
$$

We conclude that

$$
J(x, u) \ll 1 \quad \text { for } u \geq 1 .
$$

The proposition follows from (3.25), (3.26), and (3.27).

3.5. The second error estimate. In the case when $k=-1 / 2$ we must keep track of the dependence on $x$.

Proposition 3.9. Suppose that $k=-1 / 2$. For $a>0$ we have

$$
I(x, a) \ll \sqrt{x} \times \begin{cases}a^{-\frac{1}{2}}(\log (1 / a)+1) & \text { if } a \leq 1 \\ a^{-1} & \text { if } a \geq 1 .\end{cases}
$$

Proof. As before, we estimate the term involving $\cos (u \operatorname{ch} \xi)$ and we write

$$
\begin{aligned}
\int_{0}^{\infty} \cos (2 t \xi) \cos (u \operatorname{ch} \xi) d \xi \\
=\frac{1}{2 t} \sin (2 t T) \cos (u \operatorname{ch} T)+\frac{u}{2 t} \int_{0}^{T} \operatorname{sh} \xi \sin (2 t \xi) \sin (u \operatorname{ch} \xi) d \xi+R_{T}(u, t),
\end{aligned}
$$

with $R_{T}(u, t) \ll u^{-1 / 2} e^{-T / 2}$. Recalling (3.8) and (3.9), we have

$$
\int_{0}^{x} g(t) \int_{a}^{\infty} u^{-\frac{3}{2}} R_{T}(u, t) d u d t \ll a^{-1} x^{\frac{5}{2}} e^{-T / 2} \rightarrow 0 \quad \text { as } T \rightarrow \infty
$$

and, by the first derivative estimate,

$$
\int_{0}^{x} \frac{g(t)}{t} \sin (2 t T) d t \int_{a}^{\infty} u^{-\frac{3}{2}} \cos (u \operatorname{ch} T) d u \ll a^{-\frac{3}{2}} x^{\frac{3}{2}} e^{-T} \rightarrow 0 \quad \text { as } T \rightarrow \infty .
$$

Our goal is to estimate

$$
J(x, a):=\int_{0}^{\infty} \operatorname{sh} \xi G_{x}(\xi) H(a, \xi) d \xi
$$

where

$$
G_{x}(\xi):=\int_{0}^{x} \frac{g(t)}{t} \sin (2 t \xi) d t
$$

and

$$
H(a, \xi):=\int_{a}^{\infty} u^{-\frac{1}{2}} \sin (u \operatorname{ch} \xi) d u .
$$

The integral defining $J(x, a)$ converges by an argument as in the proof of Proposition 3.8 . 
Recalling (3.8) and (3.14), the first derivative estimate gives

$$
G_{x}(\xi) \ll \frac{\sqrt{x}}{\xi} .
$$

For a better estimate, we integrate by parts in (3.28) to get

$$
G_{x}(\xi)=\frac{\pi \Gamma\left(\frac{5}{4}\right)^{2}}{2 \xi}-\frac{g(x)}{x} \frac{\cos (2 x \xi)}{2 \xi}+\frac{1}{2 \xi} \int_{0}^{x}\left(\frac{g(t)}{t}\right)^{\prime} \cos (2 t \xi) d t .
$$

We estimate the third term in two ways. Taking absolute values and using (3.14), it is $\ll \sqrt{x} / \xi$; by (3.12) and Lemma 3.5 it is $\ll 1 / \xi^{2}$. Thus

$$
\frac{1}{\xi} \int_{0}^{x}\left(\frac{g(t)}{t}\right)^{\prime} \cos (2 t \xi) d t \ll \min \left(\frac{\sqrt{x}}{\xi}, \frac{1}{\xi^{2}}\right) .
$$

We also need two estimates for $H(a, \xi)$. The first derivative estimate applied to (3.29) gives

$$
H(a, \xi) \ll \frac{1}{\sqrt{a} \operatorname{ch} \xi},
$$

while integrating (3.29) by parts and applying the first derivative estimate gives

$$
H(a, \xi)=\frac{\cos (a \operatorname{ch} \xi)}{\sqrt{a} \operatorname{ch} \xi}+O\left(\frac{1}{a^{\frac{3}{2}}(\operatorname{ch} \xi)^{2}}\right) .
$$

First suppose that $a \leq 1$. Write

$$
J(x, a)=J_{1}(x, a)+J_{2}(x, a)=\left(\int_{0}^{1 / a}+\int_{1 / a}^{\infty}\right) \operatorname{sh} \xi G_{x}(\xi) H(a, \xi) d \xi .
$$

By (3.30) and (3.33) we have

$$
J_{1}(x, a) \ll \sqrt{\frac{x}{a}}\left(\int_{0}^{1}+\int_{1}^{1 / a}\right) \frac{\operatorname{th} \xi}{\xi} d \xi \ll \sqrt{\frac{x}{a}}(1+\log (1 / a)) .
$$

By (3.34), (3.30), (3.31), and the second bound in (3.32) we have

$$
\begin{aligned}
J_{2}(x, a) & =\frac{1}{\sqrt{a}} \int_{1 / a}^{\infty} \operatorname{th} \xi G_{x}(\xi) \cos (a \operatorname{ch} \xi) d \xi+O\left(\frac{\sqrt{x}}{a^{\frac{3}{2}}} \int_{1 / a}^{\infty} \frac{\operatorname{sh} \xi}{\xi(\operatorname{ch} \xi)^{2}} d \xi\right) \\
& \ll \frac{1}{\sqrt{a}} \int_{1 / a}^{\infty} \frac{\operatorname{th} \xi}{\xi} \cos (a \operatorname{ch} \xi) d \xi+\frac{g(x)}{x \sqrt{a}} \int_{1 / a}^{\infty} \frac{\operatorname{th} \xi}{\xi} \cos (2 x \xi) \cos (a \operatorname{ch} \xi) d \xi+\sqrt{x / a} .
\end{aligned}
$$

Applying the first derivative estimate to each integral above, we find that $J_{2}(x, a) \ll \sqrt{x / a}$, from which it follows that

$$
J(x, a) \ll \sqrt{\frac{x}{a}}(\log (1 / a)+1) \quad \text { for } a \leq 1 .
$$

Now suppose that $a \geq 1$. Using (3.30), the contribution to $J(x, a)$ from the second term in (3.34) is

$$
a^{-\frac{3}{2}} \int_{0}^{\infty} \frac{\operatorname{sh} \xi}{(\operatorname{ch} \xi)^{2}} G_{x}(\xi) d \xi \ll a^{-\frac{3}{2}} \sqrt{x}
$$


We break the integral involving $\cos (a \operatorname{ch} \xi)$ at $1 / \sqrt{a}$. By (3.30) the contribution from the initial segment is $\ll a^{-1} \sqrt{x}$. It remains to estimate

$$
\frac{1}{\sqrt{a}} \int_{1 / \sqrt{a}}^{\infty} \operatorname{th} \xi G_{x}(\xi) \cos (a \operatorname{ch} \xi) d \xi
$$

which we break into three terms using (3.31). For the first two terms the first derivative estimate gives

$$
\begin{gathered}
\frac{1}{\sqrt{a}} \int_{1 / \sqrt{a}}^{\infty} \frac{\operatorname{th} \xi}{\xi} \cos (a \operatorname{ch} \xi) d \xi \ll \frac{1}{a}, \\
\frac{g(x)}{x \sqrt{a}} \int_{1 / \sqrt{a}}^{\infty} \frac{\operatorname{th} \xi}{\xi} \cos (2 x \xi) \cos (a \operatorname{ch} \xi) d \xi \ll \frac{\sqrt{x}}{a} .
\end{gathered}
$$

The remaining term,

$$
\frac{1}{\sqrt{a}} \int_{1 / \sqrt{a}}^{\infty} \frac{\operatorname{th} \xi}{\xi} \cos (a \operatorname{ch} \xi) \int_{0}^{x}\left(\frac{g(t)}{t}\right)^{\prime} \sin (2 t \xi) d t d \xi
$$

requires more care, but we follow the estimate for the corresponding term in the proof of Proposition 3.8 using Lemma 3.5 and (3.13). The details are similar, and the contribution is $\ll \frac{\sqrt{x}}{a}$, which, together with (3.35), completes the proof.

3.6. Proof of Theorem 3.1. We give the proof for the case $k=1 / 2$, as the other case is analogous. By Proposition 3.8 we have

$$
I(x, a) \ll \min \left(1, a^{1 / 2} \log (1 / a)\right) \ll_{\delta} a^{\delta} \quad \text { for any } 0 \leq \delta<1 / 2 .
$$

So by (3.5) and (3.10) we have

$$
n_{\nu} \sum_{r_{j}} \frac{\left|a_{j}(n)\right|^{2}}{\operatorname{ch} \pi r_{j}} h_{x}\left(r_{j}\right)=\frac{x^{\frac{3}{2}}}{3 \pi^{2}}+O_{\delta}\left(x^{\frac{1}{2}}+n^{\frac{1}{2}+\delta} \sum_{c>0} \frac{|S(n, n, c, \nu)|}{c^{3 / 2+\delta}}\right) .
$$

Setting $\delta=\beta-1 / 2$, with $\beta$ as in (3.1), this becomes

$$
n_{\nu} \sum_{r_{j}} \frac{\left|a_{j}(n)\right|^{2}}{\operatorname{ch} \pi r_{j}} h_{x}\left(r_{j}\right)=\frac{x^{\frac{3}{2}}}{3 \pi^{2}}+O\left(x^{\frac{1}{2}}+n^{\beta+\epsilon}\right) .
$$

The function $h_{x}(r)$ (recall (3.6) ) is a smooth approximation to the characteristic function of $[0, x]$. We recall some properties from $[\mathrm{Ku}$, (5.4)-(5.7)] for $x \geq 1$ :

$$
\begin{array}{ll}
h_{x}(r)=\frac{2}{\pi} \arctan \left(e^{\pi x-\pi r}\right)+O\left(e^{-\pi r}\right) & \text { for } r \geq 1, \\
h_{x}(r)=1+O\left(x^{-3}+e^{-\pi r}\right) & \text { for } 1 \leq r \leq x-\log x, \\
h_{x}(r) \ll e^{-\pi(r-x)} & \text { for } r \geq x+\log x, \\
0<h_{x}(r)<1 & \text { for } r \geq 0 .
\end{array}
$$

For $x \geq 1$ and $0 \leq r \leq x$ we bound $h_{x}(r)$ uniformly from below as follows. If $r \geq 1$ we have

$$
h_{x}(r)=4 \operatorname{ch} \pi r \int_{0}^{x} \frac{\operatorname{sh} \pi t}{\operatorname{ch} 2 \pi r+\operatorname{ch} 2 \pi t} d t \geq \frac{2 \operatorname{ch} \pi r}{\operatorname{ch} 2 \pi r} \int_{0}^{r} \operatorname{sh} \pi t d t>\frac{1}{4},
$$


while if $r \leq 1$ we have

$$
h_{x}(r) \geq 4 \operatorname{ch} \pi r \int_{0}^{1} \frac{\operatorname{sh} \pi t}{\operatorname{ch} 2 \pi r+\operatorname{ch} 2 \pi t} d t \geq 4 \int_{0}^{1} \frac{\operatorname{sh} \pi t}{\operatorname{ch} 2 \pi+\operatorname{ch} 2 \pi t} d t>\frac{3}{100} .
$$

Similarly, we have $h_{x}(r)>2 / 5$ for $r \in i[0,1 / 4]$.

Set

$$
A(x):=n_{\nu} \sum_{\substack{r_{j} \leq x \\ \text { or } r_{j} \in i \mathbb{R}}} \frac{\left|a_{j}(n)\right|^{2}}{\operatorname{ch} \pi r_{j}}
$$

Then

$$
A(x) \ll n_{\nu} \sum_{\substack{r_{j} \leq x \\ \text { or } r_{j} \in i \mathbb{R}}} \frac{\left|a_{j}(n)\right|^{2}}{\operatorname{ch} \pi r_{j}} h_{x}\left(r_{j}\right) \ll n_{\nu} \sum_{r_{j}} \frac{\left|a_{j}(n)\right|^{2}}{\operatorname{ch} \pi r_{j}} h_{x}\left(r_{j}\right)
$$

which, together with (3.36), gives

$$
A(x) \ll x^{\frac{3}{2}}+n^{\beta+\epsilon} .
$$

Let $A^{*}(x)$ denote the sum (3.40) restricted to $1 \leq r_{j} \leq x$. By (3.41) with $x=1$ we have $A(x)=A^{*}(x)+O\left(n^{\beta+\epsilon}\right)$, so in what follows we work with $A^{*}(x)$.

Assume that $x \geq 2$ and set $X=x+2 \log x$ so that $X-\log X \geq x$. Then by (3.37) there exist $c_{1}, c_{2}>0$ such that

$$
n_{\nu} \sum_{1 \leq r_{j}} \frac{\left|a_{j}(n)\right|^{2}}{\operatorname{ch} \pi r_{j}} h_{X}\left(r_{j}\right) \geq A^{*}(x)\left(1-\frac{c_{1}}{x^{3}}\right)-c_{2} n_{\nu} \sum_{1 \leq r_{j} \leq x} \frac{\left|a_{j}(n)\right|^{2}}{\operatorname{ch} \pi r_{j}} e^{-\pi r_{j}} .
$$

On the other hand, (3.36) gives

$$
n_{\nu} \sum_{1 \leq r_{j}} \frac{\left|a_{j}(n)\right|^{2}}{\operatorname{ch} \pi r_{j}} h_{X}\left(r_{j}\right)=\frac{x^{\frac{3}{2}}}{3 \pi^{2}}+O\left(x^{\frac{1}{2}} \log x+n^{\beta+\epsilon}\right) .
$$

By (3.41) we have

$$
n_{\nu} \sum_{1 \leq r_{j} \leq x} \frac{\left|a_{j}(n)\right|^{2}}{\operatorname{ch} \pi r_{j}} e^{-\pi r_{j}}=n_{\nu} \sum_{\ell=1}^{\lfloor x\rfloor} \sum_{\ell \leq r_{j} \leq \ell+1} \frac{\left|a_{j}(n)\right|^{2}}{\operatorname{ch} \pi r_{j}} e^{-\pi r_{j}} \ll \sum_{\ell=1}^{\infty} e^{-\pi \ell}\left(\ell^{\frac{3}{2}}+n^{\beta+\epsilon}\right) \ll n^{\beta+\epsilon},
$$

so that

$$
A^{*}(x) \leq \frac{x^{\frac{3}{2}}}{3 \pi^{2}}+O\left(x^{\frac{1}{2}} \log x+n^{\beta+\epsilon}\right) .
$$

Similarly, replacing $x$ by $x-\log x$ in (3.36) and using (3.38), (3.39) gives

$$
A^{*}(x) \geq \frac{x^{\frac{3}{2}}}{3 \pi^{2}}+O\left(x^{\frac{1}{2}} \log x+n^{\beta+\epsilon}\right)+O\left(n_{\nu} \sum_{r_{j}>x} \frac{\left|a_{j}(n)\right|^{2}}{\operatorname{ch} \pi r_{j}} e^{-\pi\left(r_{j}-x+\log x\right)}\right) .
$$

Arguing as in (3.42), the error term is $\ll x^{-\frac{3}{2}}+n^{\beta+\epsilon} x^{-3}$, from which we obtain

$$
A^{*}(x) \geq \frac{x^{\frac{3}{2}}}{3 \pi^{2}}+O\left(x^{\frac{1}{2}} \log x+n^{\beta+\epsilon}\right) .
$$

Equations (3.43) and (3.44) together give Theorem 3.1. 


\section{The Kuznetsov trace formula}

We require a variant of Kuznetsov's formula [Ku, Theorem 1], which relates weighted sums of the Kloosterman sums $S(m, n, c, \chi)$ on one side to spectral data on the other side (in this case, weighted sums of coefficients of Maass cusp forms). In [P2], Proskurin generalized Kuznetsov's theorem to any weight $k$ and multiplier system $\nu$, but only for the case $m, n>0$. Here we treat the mixed sign case when $k=1 / 2$ and $\nu=\chi$; the proof follows the same lines. Blomer [B] has recorded this formula for twists of the theta-multiplier by a Dirichlet character; we provide a sketch of the proof in the present case since there are some details which are unique to this situation.

Suppose that $\phi:[0, \infty) \rightarrow \mathbb{C}$ is four times continuously differentiable and satisfies

$$
\phi(0)=\phi^{\prime}(0)=0, \quad \phi^{(j)}(x) \ll_{\epsilon} x^{-2-\epsilon} \quad(j=0, \ldots, 4) \quad \text { as } x \rightarrow \infty
$$

for some $\epsilon>0$. Define

$$
\check{\phi}(r):=\operatorname{ch} \pi r \int_{0}^{\infty} K_{2 i r}(u) \phi(u) \frac{d u}{u} .
$$

We state the analogue of the main result of [P2] in this case.

Theorem 4.1. Suppose that $\phi$ satisfies the conditions (4.1). As in (2.23) let $\rho_{j}(n)$ denote the coefficients of an orthornomal basis $\left\{u_{j}\right\}$ for $\mathcal{S}_{1 / 2}(1, \chi)$ with spectral parameters $r_{j}$. If $m>0$ and $n<0$ then

$$
\sum_{c>0} \frac{S(m, n, c, \chi)}{c} \phi\left(\frac{4 \pi \sqrt{\widetilde{m}|\widetilde{n}|}}{c}\right)=8 \sqrt{i} \sqrt{\widetilde{m}|\widetilde{n}|} \sum_{r_{j}} \frac{\overline{\rho_{j}(m)} \rho_{j}(n)}{\operatorname{ch} \pi r_{j}} \check{\phi}\left(r_{j}\right) .
$$

The proof involves evaluating the inner product of Poincaré series in two ways. Let $\tau=x+i y \in \mathbb{H}$ and $s=\sigma+i t \in \mathbb{C}$. Let $k \in \mathbb{R}$ and let $\nu$ be a multiplier system for $\Gamma$ in weight $k$. For $m>0$ the Poincaré series $\mathcal{U}_{m}(\tau, s, k, \nu)$ is defined by

$$
\mathcal{U}_{m}(\tau, s, k, \nu):=\sum_{\gamma \in \Gamma_{\infty} \backslash \Gamma} \overline{\nu(\gamma)} j(\gamma, \tau)^{-k} \operatorname{Im}(\gamma \tau)^{s} e\left(m_{\nu} \gamma \tau\right), \quad \sigma>1 .
$$

This function satisfies the transformation law

$$
\left.\mathcal{U}_{m}(\cdot, s, k, \nu)\right|_{k} \gamma=\nu(\gamma) \mathcal{U}_{m}(\cdot, s, k, \nu) \quad \text { for all } \gamma \in \Gamma
$$

and has Fourier expansion (see equation (15) of [P2])

$$
\mathcal{U}_{m}(\tau, s, k, \nu)=y^{s} e\left(m_{\nu} \tau\right)+y^{s} \sum_{\ell \in \mathbb{Z}} \sum_{c>0} \frac{S(m, \ell, c, \nu)}{c^{2 s}} B\left(c, m_{\nu}, \ell_{\nu}, y, s, k\right) e\left(\ell_{\nu} x\right)
$$

where

$$
B\left(c, m_{\nu}, \ell_{\nu}, y, s, k\right)=y \int_{-\infty}^{\infty} e\left(\frac{-m_{\nu}}{c^{2} y(u+i)}\right)\left(\frac{u+i}{|u+i|}\right)^{-k} e\left(-\ell_{\nu} y u\right) \frac{d u}{y^{2 s}\left(u^{2}+1\right)^{s}} .
$$

In the next two lemmas we will obtain expressions for the inner product

$$
\left\langle\mathcal{U}_{m}\left(\cdot, s_{1}, \frac{1}{2}, \chi\right), \overline{\mathcal{U}_{1-n}\left(\cdot, s_{2},-\frac{1}{2}, \bar{\chi}\right)}\right\rangle .
$$

The first lemma is stated in such a way that symmetry may be exploited in its proof. 
Lemma 4.2. Suppose that $(k, \nu)$ is one of the pairs $(1 / 2, \chi)$ or $(-1 / 2, \bar{\chi})$. Suppose that $m>0, n<0$. Then for $\operatorname{Re} s_{1}>1$, $\operatorname{Re} s_{2}>1$ we have

$$
\begin{aligned}
& \left\langle\mathcal{U}_{m}\left(\cdot, s_{1}, k, \nu\right), \overline{\mathcal{U}_{1-n}\left(\cdot, s_{2},-k, \bar{\nu}\right)}\right\rangle \\
& =i^{-k} 2^{3-s_{1}-s_{2}} \pi\left(\frac{m_{\nu}}{\left|n_{\nu}\right|}\right)^{\frac{s_{2}-s_{1}}{2}} \frac{\Gamma\left(s_{1}+s_{2}-1\right)}{\Gamma\left(s_{1}-k / 2\right) \Gamma\left(s_{2}+k / 2\right)} \sum_{c>0} \frac{S(m, n, c, \nu)}{c^{s_{1}+s_{2}}} K_{s_{1}-s_{2}}\left(\frac{4 \pi \sqrt{m_{\nu}\left|n_{\nu}\right|}}{c}\right) .
\end{aligned}
$$

Proof of Lemma 4.2. We will prove (4.6) under the assumption $\operatorname{Re} s_{2}>\operatorname{Re} s_{1}$. Recall the definitions (2.25), (2.5), and (2.13), and recall that

$$
(1-n)_{\bar{\nu}}=-n_{\nu}
$$

Replacing $\gamma$ by $-\gamma^{-1}$ in (2.25) and using $\nu(-I)=e^{-\pi i k}$, we find that

$$
S(m, n, c, \nu)=e^{\pi i k} S(1-n, 1-m, c, \bar{\nu}) .
$$

The case $\operatorname{Re} s_{1}>\operatorname{Re} s_{2}$ then follows since both sides of the putative identity are invariant under the changes $s_{1} \leftrightarrow s_{2}, k \leftrightarrow-k, \nu \leftrightarrow \bar{\nu}, m \leftrightarrow 1-n$ (see [DL, (10.27.3)]). The case $\operatorname{Re} s_{1}=\operatorname{Re} s_{2}$ follows by continuity.

Let $I_{m, n}\left(s_{1}, s_{2}\right)$ denote the inner product on the left-hand side of (4.6). Unfolding using (4.4) and (4.3), we find that

$$
\begin{aligned}
I_{m, n}\left(s_{1}, s_{2}\right) & =\int_{\Gamma_{\infty} \backslash \mathbb{H}} \mathcal{U}_{m}\left(\tau, s_{1}, k, \nu\right)(\operatorname{Im} \tau)^{s_{2}} e\left(-n_{\nu}\right) d \mu \\
& =\int_{0}^{\infty} y^{s_{1}+s_{2}-2} \sum_{c>0} \frac{S(m, n, c, \nu)}{c^{2 s_{1}}} B\left(c, m_{\nu}, n_{\nu}, y, s_{1}, k\right) e^{2 \pi n_{\nu} y} d y
\end{aligned}
$$

By [P2, (17)] we have

$$
B\left(c, m_{\nu}, n_{\nu}, y, s_{1}, k\right) \ll_{s_{1}} y^{1-2 \operatorname{Re} s_{1}} e^{-\pi\left|n_{\nu}\right| y} .
$$

Therefore the integral in (4.7) is majorized by the convergent (since $\operatorname{Re} s_{2}>\operatorname{Re} s_{1}$ ) integral

$$
\int_{0}^{\infty} y^{\operatorname{Re} s_{2}-\operatorname{Re} s_{1}-1} e^{3 \pi n_{\nu} y} d y .
$$

Interchanging the integral and sum in (4.7) and using (4.5), we find that

$$
\begin{aligned}
I_{m, n}\left(s_{1}, s_{2}\right)=\sum_{c>0} \frac{S(m, n, c, \nu)}{c^{2 s_{1}}} \int_{-\infty}^{\infty}\left(\frac{u+i}{|u+i|}\right)^{-k}\left(u^{2}+1\right)^{-s_{1}} & \\
& \times\left(\int_{0}^{\infty} y^{s_{2}-s_{1}-1} e\left(\frac{-m_{\nu}}{c^{2} y(u+i)}-n_{\nu} y u\right) e^{2 \pi n_{\nu} y} d y\right) d u .
\end{aligned}
$$

Setting $w=2 \pi n_{\nu}(i u-1) y$ and $a=4 \pi \sqrt{m_{\nu}\left|n_{\nu}\right|} / c$, the inner integral becomes

$$
\left(2 \pi n_{\nu}(i u-1)\right)^{s_{1}-s_{2}} \int_{0}^{(1-i u) \infty} \exp \left(-w-\frac{a^{2}}{4 w}\right) \frac{d w}{w^{s_{1}-s_{2}+1}} .
$$

We may shift the path of integration to the positive real axis since

$$
\int_{T}^{(1-i u) T} \exp \left(-w-\frac{a^{2}}{4 w}\right) \frac{d w}{w^{s_{1}+s_{2}+1}} \ll T^{-\operatorname{Re}\left(s_{1}-s_{2}\right)} e^{-T} \quad \text { as } T \rightarrow \infty .
$$


Using the integral representation [DL, (10.32.10)] for the $K$-Bessel function, we find that the inner integral is equal to

$$
2\left(\frac{m_{\nu}}{\left|n_{\nu}\right|}\right)^{\frac{s_{2}-s_{1}}{2}}(1-i u)^{s_{1}-s_{2}} c^{s_{1}-s_{2}} K_{s_{1}-s_{2}}\left(\frac{4 \pi \sqrt{m_{\nu}\left|n_{\nu}\right|}}{c}\right) .
$$

It follows that

$$
\begin{aligned}
I_{m, n}\left(s_{1}, s_{2}\right)=2\left(\frac{m_{\nu}}{\left|n_{\nu}\right|}\right)^{\frac{s_{2}-s_{1}}{2}} \sum_{c>0} \frac{S(m, n, c, \nu)}{c^{s_{1}+s_{2}}} & K_{s_{1}-s_{2}}\left(\frac{4 \pi \sqrt{m_{\nu}\left|n_{\nu}\right|}}{c}\right) \\
& \times \int_{-\infty}^{\infty}\left(\frac{u+i}{|u+i|}\right)^{-k}\left(u^{2}+1\right)^{-s_{1}}(1-i u)^{s_{1}-s_{2}} d u .
\end{aligned}
$$

Lemma 4.2 follows after using [DL, (5.12.8)] to evaluate the integral.

Recall the definition

$$
\Lambda\left(s_{1}, s_{2}, r\right)=\Gamma\left(s_{1}-\frac{1}{2}-i r\right) \Gamma\left(s_{1}-\frac{1}{2}+i r\right) \Gamma\left(s_{2}-\frac{1}{2}-i r\right) \Gamma\left(s_{2}-\frac{1}{2}+i r\right) .
$$

Lemma 4.3. Suppose that $\operatorname{Re} s_{1}>1, \operatorname{Re} s_{2}>1$. As in (2.23) let $\rho_{j}(n)$ and $r_{j}$ denote the coefficients and spectral parameters of an orthornomal basis $\left\{u_{j}\right\}$ for $\mathcal{S}_{1 / 2}(1, \chi)$. If $m>0$ and $n<0$ then

$$
\left\langle\mathcal{U}_{m}\left(\cdot, s_{1}, \frac{1}{2}, \chi\right), \overline{\mathcal{U}_{1-n}\left(\cdot, s_{2},-\frac{1}{2}, \bar{\chi}\right)}\right\rangle=\frac{\left(4 \pi m_{\chi}\right)^{1-s_{1}}\left(4 \pi\left|n_{\chi}\right|\right)^{1-s_{2}}}{\Gamma\left(s_{1}-1 / 4\right) \Gamma\left(s_{2}+1 / 4\right)} \sum_{r_{j}} \overline{\rho_{j}(m)} \rho_{j}(n) \Lambda\left(s_{1}, s_{2}, r_{j}\right) .
$$

Proof of Lemma 4.3. In this situation there are no Eisenstein series. So for any $f_{1}, f_{2} \in$ $\mathcal{L}_{\frac{1}{2}}(1, \chi)$ we have the Parseval identity [P2, (27)]

$$
\left\langle f_{1}, f_{2}\right\rangle=\sum_{r_{j}}\left\langle f_{1}, u_{j}\right\rangle \overline{\left\langle f_{2}, u_{j}\right\rangle}
$$

By [P2, (32)] we have

$$
\left\langle\mathcal{U}_{m}\left(z, s_{1}, \frac{1}{2}, \chi\right), u_{j}\right\rangle=\overline{\rho_{j}(m)}\left(4 \pi m_{\chi}\right)^{1-s_{1}} \frac{\Gamma\left(s_{1}-\frac{1}{2}-i r_{j}\right) \Gamma\left(s_{1}-\frac{1}{2}+i r_{j}\right)}{\Gamma\left(s_{1}-\frac{1}{4}\right)} .
$$

Unfolding as in the last lemma using the definition (4.3) for $\mathcal{U}_{1-n}$ and the Fourier expansion (2.23) for $u_{j}$ gives

$$
\begin{aligned}
\overline{\left\langle\overline{\mathcal{U}_{1-n}\left(\cdot, s_{2},-\frac{1}{2}, \bar{\chi}\right)}, u_{j}\right\rangle} & =\left\langle u_{j}, \overline{\mathcal{U}_{1-n}\left(\cdot, s_{2},-\frac{1}{2}, \bar{\chi}\right)}\right\rangle \\
& =\rho_{j}(n) \int_{0}^{\infty} y^{s_{2}-2} W_{-\frac{1}{4}, i r_{j}}\left(4 \pi\left|n_{\chi}\right| y\right) e^{2 \pi n_{\chi} y} d y .
\end{aligned}
$$

Using [DL, (13.23.4) and (16.2.5)] the latter expression becomes

$$
\rho_{j}(n)\left(4 \pi\left|n_{\chi}\right|\right)^{1-s_{2}} \frac{\Gamma\left(s_{2}-\frac{1}{2}-i r_{j}\right) \Gamma\left(s_{2}-\frac{1}{2}+i r_{j}\right)}{\Gamma\left(s_{2}+\frac{1}{4}\right)} .
$$

The lemma follows from this together with (4.8) and (4.9).

We are now ready to prove Theorem 4.1, Recall the notation $\tilde{n}=n_{\chi}$. 
Proof of Theorem 4.1. Equating the right-hand sides of Lemmas 4.2 (for $k=1 / 2$ ) and 4.3 and setting

$$
s_{1}=\sigma+\frac{i t}{2}, \quad s_{2}=\sigma-\frac{i t}{2}
$$

we obtain (for $\sigma>1$ )

$$
\begin{aligned}
i^{-\frac{1}{2}} 2^{3-2 \sigma} \pi \Gamma(2 \sigma-1) \sum_{c>0} & \frac{S(m, n, c, \chi)}{c^{2 \sigma}} K_{i t}\left(\frac{4 \pi \sqrt{\widetilde{m}|\widetilde{n}|}}{c}\right) \\
& =(4 \pi \widetilde{m})^{1-\sigma}(4 \pi|\widetilde{n}|)^{1-\sigma} \sum_{r_{j}} \frac{\rho_{j}(m)}{\rho_{j}}(n) \Lambda\left(\sigma+\frac{i t}{2}, \sigma-\frac{i t}{2}, r_{j}\right) .
\end{aligned}
$$

We justify the substitution of $\sigma=1$ in (4.10). By [DL, (10.45.7)] we have

$$
K_{i t}(x) \ll(t \operatorname{sh} \pi t)^{-1 / 2} \quad \text { as } x \rightarrow 0 .
$$

Using (2.30) we see that the left side of (4.10) converges absolutely uniformly for $\sigma \in[1,2]$. For the right hand side we use the inequality $\left|\rho_{j}(m) \rho_{j}(n)\right| \leq\left|\rho_{j}(m)\right|^{2}+\left|\rho_{j}(n)\right|^{2}$. The argument which follows Lemma 3.2 shows that the right side converges absolutely uniformly for $\sigma \in[1,2]$.

Using (3.4), we find that

$$
i^{-\frac{1}{2}} \sum_{c>0} \frac{S(m, n, c, \chi)}{c^{2}} K_{i t}\left(\frac{4 \pi \sqrt{\widetilde{m}|\widetilde{n}|}}{c}\right)=\frac{\pi}{2} \sum_{r_{j}} \frac{\overline{\rho_{j}(m)} \rho_{j}(n)}{\operatorname{ch} \pi\left(\frac{t}{2}-r_{j}\right) \operatorname{ch} \pi\left(\frac{t}{2}+r_{j}\right)} .
$$

Letting $\phi$ be a function satisfying the conditions (4.1), multiply both sides of (4.11) by

$$
\frac{2}{\pi^{2}} t \operatorname{sh} \pi t \int_{0}^{\infty} K_{i t}(u) \phi(u) \frac{d u}{u^{2}}
$$

and integrate from 0 to $\infty$. We apply the Kontorovich-Lebedev transform $([\mathrm{P} 1$, (35)] or [DL, $(10.43 .30-31)])$

$$
\frac{2}{\pi^{2}} \int_{0}^{\infty} K_{i t}(x) t \operatorname{sh} \pi t\left(\int_{0}^{\infty} K_{i t}(u) \phi(u) \frac{d u}{u^{2}}\right) d t=\frac{\phi(x)}{x}
$$

to the left-hand side of (4.11) and the transform [P1, (39)]

$$
\int_{0}^{\infty} \frac{t \operatorname{sh} \pi t}{\operatorname{ch} \pi\left(\frac{t}{2}+r\right) \operatorname{ch} \pi\left(\frac{t}{2}-r\right)}\left(\int_{0}^{\infty} K_{i t}(u) \phi(u) \frac{d u}{u^{2}}\right) d t=\frac{2}{\operatorname{ch} \pi r} \check{\phi}(r)
$$

(recalling the definition (4.2)) to the right-hand side. Then (4.11) becomes

$$
\frac{i^{-1 / 2}}{4 \pi \sqrt{\widetilde{m}|\widetilde{n}|}} \sum_{c>0} \frac{S(m, n, c, \chi)}{c} \phi\left(\frac{4 \pi \sqrt{\widetilde{m}|\widetilde{n}|}}{c}\right)=\frac{2}{\pi} \sum_{r_{j}} \frac{\overline{\rho_{j}(m)} \rho_{j}(n)}{\operatorname{ch} \pi r_{j}} \check{\phi}\left(r_{j}\right),
$$

and Theorem 4.1 follows. 


\section{A THETA LIFT FOR MAASS CUSP FORMS}

In this section we construct a version of the Shimura correspondence as in [Sa, §3] and [KS, $\S 4]$ for Maass forms of weight $1 / 2$ on $\Gamma_{0}(N)$ with the eta multiplier twisted by a Dirichlet character.

Throughout this section, $N \equiv 1 \bmod 24$ is a positive integer and $\psi$ is an even Dirichlet character modulo $N$. When working with the Shimura correspondence and the Hecke operators, it is convenient to write the Fourier expansion of $G \in \mathcal{S}_{\frac{1}{2}}(N, \psi \chi, r)$ as

$$
G(\tau)=\sum_{n \equiv 1(24)} a(n) W_{\frac{\operatorname{sgn}(n)}{4}, i r}\left(\frac{\pi|n| y}{6}\right) e\left(\frac{n x}{24}\right) .
$$

When $G=u_{j}$ as in (2.23) we have the relation

$$
\rho_{j}\left(\frac{n+23}{24}\right)=a(n)
$$

For each $t \equiv 1 \bmod 24$, the following theorem gives a map

$$
S_{t}: \mathcal{S}_{\frac{1}{2}}(N, \psi \chi, r) \rightarrow \mathcal{S}_{0}\left(6 N, \psi^{2}, 2 r\right) .
$$

We will only apply this theorem in the case $N=1$. The proof in the case $N=t=1$ is simpler than the general proof given below; we must work in this generality since the proof in the case $t>1$ requires the map $S_{1}$ on $\Gamma_{0}(t)$ forms.

Theorem 5.1. Let $N \equiv 1 \bmod 24$ be squarefree. Suppose that $G \in \mathcal{S}_{\frac{1}{2}}(N, \psi \chi, r)$ with $r \neq i / 4$ has Fourier expansion (5.1). Let $t \equiv 1 \bmod 24$ be a squarefree positive integer and define coefficients $b_{t}(n)$ by the relation

$$
\sum_{n=1}^{\infty} \frac{b_{t}(n)}{n^{s}}=L\left(s+1, \psi\left(\frac{t}{\bullet}\right)\right) \sum_{n=1}^{\infty}\left(\frac{12}{n}\right) \frac{a\left(t n^{2}\right)}{n^{s-\frac{1}{2}}},
$$

where $L(s, \psi)$ is the usual Dirichlet L-function. Then the function $S_{t}(G)$ defined by

$$
\left(S_{t} G\right)(\tau):=\sum_{n=1}^{\infty} b_{t}(n) W_{0,2 i r}(4 \pi n y) \cos (2 \pi n x)
$$

is an even Maass cusp form in $\mathcal{S}_{0}\left(6 N, \psi^{2}, 2 r\right)$.

An important property of the Shimura correspondence is Hecke equivariance. Since we only need this fact for $N=1$, we state it only in that case.

Corollary 5.2. Suppose that $G \in \mathcal{S}_{\frac{1}{2}}(1, \chi, r)$ with $r \neq i / 4$ and that $t \equiv 1 \bmod 24$ is a squarefree positive integer. Then for any prime $p \geq 5$ we have

$$
T_{p} S_{t}(G)=\left(\frac{12}{p}\right) S_{t}\left(T_{p^{2}} G\right)
$$

Proof. We prove this in the case $p \nmid t$; the other case is similar, and easier. Let $G$ have coefficients $a(n)$ as in (5.1) . The coefficients $b_{t}(n)$ of $S_{t}(G)$ are given by

$$
b_{t}(n)=\sum_{j k=n}\left(\frac{t}{j}\right)\left(\frac{12}{k}\right) \frac{\sqrt{k}}{j} a\left(t k^{2}\right) \text {, }
$$


and the coefficients $A(n)$ of $T_{p^{2}} G$ are given by

$$
A(n)=p a\left(p^{2} n\right)+p^{-\frac{1}{2}}\left(\frac{12 n}{p}\right) a(n)+p^{-1} a\left(n / p^{2}\right) .
$$

We must show that

$$
p^{\frac{1}{2}} b_{t}(p n)+p^{-\frac{1}{2}} b_{t}(n / p)=\left(\frac{12}{p}\right) \sum_{j k=n}\left(\frac{t}{j}\right)\left(\frac{12}{k}\right) \frac{\sqrt{k}}{j} A\left(t k^{2}\right) .
$$

Writing $n=p^{\alpha} n^{\prime}$ with $p \nmid n^{\prime}$, the left-hand side of (5.3) equals

$$
p^{-\frac{1}{2}} \sum_{\ell=0}^{\alpha+1} S_{\ell}+p^{\frac{1}{2}} \sum_{\ell=0}^{\alpha-1} S_{\ell}, \quad \text { where } S_{\ell}=p^{-\alpha}\left(\frac{t}{p}\right)^{\alpha+1-\ell}\left(\frac{12}{p}\right)^{\ell} p^{\frac{3 \ell}{2}} \sum_{j k=n^{\prime}}\left(\frac{t}{j}\right)\left(\frac{12}{k}\right) \frac{\sqrt{k}}{j} a\left(t p^{2 \ell} k^{2}\right) .
$$

A computation shows that the right-hand side of (5.3) equals

$$
p^{-\frac{1}{2}} \sum_{\ell=0}^{\alpha} S_{\ell+1}+p^{-\frac{1}{2}} S_{0}+p^{\frac{1}{2}} \sum_{\ell=1}^{\alpha} S_{\ell-1}
$$

and the corollary follows.

Using Theorem 5.1 we can rule out the existence of exceptional eigenvalues in $\mathcal{S}_{1 / 2}(1, \chi)$ and obtain a lower bound on the second smallest eigenvalue $\lambda_{1}=\frac{1}{4}+r_{1}^{2}$ (we note that Bruggeman [Br1, Theorem 2.15] obtained $\lambda_{1}>\frac{1}{4}$ using different methods). Theorem 5.1 shows that $2 r_{1}$ is bounded below by the smallest spectral parameter for $\mathcal{S}_{0}(6, \mathbf{1})$. Huxley $\left[\mathrm{H1}, \mathrm{H2}\right.$ studied the problem of exceptional eigenvalues in weight 0 for subgroups of $\mathrm{SL}_{2}(\mathbb{Z})$ whose fundamental domains are "hedgehog" shaped. On page 247 of [H1] we find a lower bound which, for $N=6$, gives $2 r_{1}>0.4$. Computations of Strömberg [LMF] suggest (since $2 r_{1}$ corresponds to an even Maass cusp form) that $2 r_{1} \approx 3.84467$. Using work of Booker and Strömbergsson [BS] on Selberg's eigenvalue conjecture we can prove the following lower bound.

Corollary 5.3. Let $\lambda_{1}=\frac{1}{4}+r_{1}^{2}$ be as above. Then $r_{1}>1.9$.

Proof. Let $\left\{\widetilde{r}_{j}\right\}$ denote the set of spectral parameters corresponding to even forms in $\mathcal{S}_{0}(6, \mathbf{1})$. We show that each $\widetilde{r}_{j}>3.8$ using the method described in [BS, Section 4] in the case of level 6 and trivial character. We are thankful to the authors of that paper for providing the numerical details of this computation. Given a suitable test function $h$ such that $h(t) \geq 0$ on $\mathbb{R}$ and $h(t) \geq 1$ on $[-3.8,3.8]$, we compute via an explicit version of the Selberg trace formula [BS, Theorem 4] that

$$
\sum_{\widetilde{r}_{j}} h\left(\widetilde{r}_{j}\right)<1
$$

so we cannot have $\widetilde{r}_{j} \leq 3.8$ for any $j$. Let

$$
f(t)=\left(\frac{\sin t / 6}{t / 6}\right)^{2} \sum_{n=0}^{2} x_{n} \cos \left(\frac{n t}{3}\right)
$$

where

$$
x_{0}=1.167099688946872, \quad x_{1}=1.735437017086616, \quad x_{2}=0.660025094420283 .
$$

With $h=f^{2}$, we compute that the sum in (5.4) is approximately 0.976 . 
The proof of Theorem 5.1 occupies the remainder of this section. We first modify the theta functions introduced by Shintani $[\mathrm{Sh}]$ and Niwa $[\mathrm{N}]$. Next, we construct the Shimura lift for $t=1$ and derive the relation (5.2) in that case. The function $S_{t}(G)$ is obtained by applying the lift $S_{1}$ to the form $G(t \tau)$. We conclude the section by showing that $S_{t}(G)$ has the desired level.

5.1. The theta functions of Shintani and Niwa. In this subsection we adopt the notation of $\left[\mathrm{N}\right.$ ] for easier comparison with that paper. Let $Q=\frac{1}{12 N}\left({ }_{-2}{ }^{-2}\right)$, and for $x, y \in \mathbb{R}^{3}$ define

$$
\langle x, y\rangle=x^{T} Q y=\frac{1}{12 N}\left(x_{2} y_{2}-2 x_{1} y_{3}-2 x_{3} y_{1}\right) .
$$

The signature of $Q$ is $(2,1)$. Let $L \subset L^{\prime} \subset L^{*}$ denote the lattices

$$
\begin{aligned}
L & =N \mathbb{Z} \oplus 12 N \mathbb{Z} \oplus 6 N \mathbb{Z}, \\
L^{\prime} & =\mathbb{Z} \oplus \quad N \mathbb{Z} \oplus 6 N \mathbb{Z}, \\
L^{*} & =\mathbb{Z} \oplus \quad \mathbb{Z} \oplus 6 \mathbb{Z} .
\end{aligned}
$$

Then $L^{*}$ is the dual lattice of $L$, and for $x, y \in L$ we have $\langle x, y\rangle \in \mathbb{Z}$ and $\langle x, x\rangle \in 2 \mathbb{Z}$. Let $f: \mathbb{R}^{3} \rightarrow \mathbb{C}$ be a Schwarz function satisfying the conditions of [N], Corollary 0$]$ for $\kappa=1$. If $\psi$ is a character $\bmod N$ and $h=\left(h_{1}, N h_{2}, 0\right) \in L^{\prime} / L$, define

$$
\psi_{1}(h):=\psi\left(h_{1}\right)\left(\frac{12}{h_{2}}\right) \text {. }
$$

For $z=u+i v$ we define

$$
\theta(z, f, h):=v^{-\frac{1}{4}} \sum_{x \in L}\left[r_{0}\left(\sigma_{z}\right) f\right](x+h)
$$

and

$$
\theta(z, f):=\sum_{h \in L^{\prime} / L} \bar{\psi}_{1}(h) \theta(z, f, h)
$$

where $\sigma \mapsto r_{0}(\sigma)$ is the Weil representation (see [N, p. 149]) and

$$
\sigma_{z}:=\left(\begin{array}{cc}
v^{\frac{1}{2}} & u v^{-\frac{1}{2}} \\
0 & v^{-\frac{1}{2}}
\end{array}\right), \quad z=u+i v \in \mathbb{H} .
$$

The following lemma gives the transformation law for $\theta(z, f)$.

Lemma 5.4. Suppose that $N \equiv 1(\bmod 24)$ and that $\sigma=\left(\begin{array}{ll}a & b \\ c & d\end{array}\right) \in \Gamma_{0}(N)$. With $\theta(z, f)$ defined as in (5.5) we have

$$
\theta(\sigma z, f)=\bar{\psi}(d)\left(\frac{N}{d}\right) \chi(\sigma)(c z+d)^{\frac{1}{2}} \theta(z, f),
$$

where $\chi$ is the eta multiplier (2.6).

In the proof of Lemma 5.4 we will encounter the quadratic Gauss sum

$$
G(a, b, c)=\sum_{x \bmod c} e\left(\frac{a x^{2}+b x}{c}\right) .
$$

With $g=(a, c)$, we have

$$
G(a, b, c)= \begin{cases}g G(a / g, b / g, c / g) & \text { if } g \mid b, \\ 0 & \text { otherwise. }\end{cases}
$$


We require the following identity relating the Dedekind sums $s(d, c)$ to these Gauss sums.

Lemma 5.5. Suppose that $(c, d)=1$ and that $k \in \mathbb{Z}$. Let $\bar{d}$ satisfy $d \bar{d} \equiv 1(\bmod c)$. Then

$$
\sqrt{12 c}\left(\frac{12}{k}\right) e\left(\frac{\bar{d}\left(k^{2}-1\right)}{24 c}\right) e^{\pi i s(d, c)}=\sum_{h \bmod 12}\left(\frac{12}{h}\right) e\left(\frac{-d\left(\frac{h^{2}-1}{24}\right)}{c}-\frac{h k}{12 c}\right) G(-6 d, d h+k, c) \text {. }
$$

Proof. First suppose that $(k, 6)=1$. If $c$ is even and $d \bar{d} \equiv 1(\bmod 2 c)$, or if $c$ is odd, then by [A, Lemma 5 and (4.1)] we have

$$
\begin{aligned}
& \sqrt{12 c}\left(\frac{12}{k}\right) e\left(\frac{\bar{d}\left(k^{2}-1\right)}{24 c}\right) e^{\pi i s(d, c)} \\
& \quad=e\left(\frac{k}{12 c}\right) \sum_{j \bmod 2 c} e\left(\frac{-3 d j^{2}-d j+j(c+k)}{2 c}\right)+e\left(\frac{-k}{12 c}\right) \sum_{j \bmod 2 c} e\left(\frac{-3 d j^{2}-d j+j(c-k)}{2 c}\right) .
\end{aligned}
$$

If $c$ is even and $d \bar{d} \not \equiv 1(\bmod 2 c)$ then, applying (5.8) with $\bar{d}$ replaced by $\bar{d}+c$, we see that (5.8) is true in this case as well. Splitting each of the sums in (5.8) into two sums by writing $j=2 x$ or $j=2 x+1$ shows that (5.8) equals the right-hand side of (5.7).

It remains to show that the right-hand side of (5.7) is zero when $\delta:=(k, 6)>1$. If $(\delta, c)>1$ then (5.6) shows that $\left(\frac{12}{h}\right) G(-6 d, d h+k, c)=0$. If $(\delta, c)=1$, write

$$
A(h)=e\left(\frac{-d\left(\frac{h^{2}-1}{24}\right)}{c}-\frac{h k}{12 c}\right) G(-6 d, d h+k, c) .
$$

If $\delta=2$ or 6 then a computation shows that, replacing $x$ by $x-\overline{2}$ in $G(-6 d, d h+k, c)$, we have $A(h)=A(h-6)$ for $(h, 6)=1$. Similarly, if $\delta=3$ and $c$ is odd then, replacing $x$ by $x+\overline{6} h$, we find that $A(h)=-A(-h)$. Finally, if $\delta=3$ and $c$ is even then, replacing $x$ by $x-\overline{3}$, we find that $A(1)=A(5)$ and $A(7)=A(11)$. In each case we find that

$$
\sum_{h \bmod 12}\left(\frac{12}{h}\right) A(h)=0 \text {. }
$$

Proof of Lemma 5.4. Since $\sigma_{z+1}=\left(\begin{array}{ll}1 & 1 \\ 0 & 1\end{array}\right) \sigma_{z}$, Proposition 0 of [N] gives

$$
\theta(z+1, f)=\sum_{h \in L^{\prime} / L} e\left(\frac{N h_{2}^{2}}{24}\right) \bar{\psi}_{1}(h) \theta(z, f, h)=e\left(\frac{1}{24}\right) \theta(z, f) .
$$

For $\sigma=\left(\begin{array}{ll}a & b \\ c & d\end{array}\right) \in \Gamma_{0}(N)$ with $c>0$ we have, by Corollary 0 of [N],

$$
\theta(\sigma z, f)=(c z+d)^{\frac{1}{2}} \sum_{k \in L^{*} / L} \theta(z, f, k) \cdot \sqrt{-i} \sum_{h \in L^{\prime} / L} \bar{\psi}_{1}(h) c(h, k)_{\sigma}
$$

where

$$
c(h, k)_{\sigma}=\frac{1}{(N c)^{\frac{3}{2}} \sqrt{12}} e\left(\frac{d\langle k, k\rangle}{2 c}\right) \sum_{r \in L / c L} e\left(\frac{a\langle h+r, h+r\rangle}{2 c}-\frac{\langle k, h+r\rangle}{c}\right) .
$$

For $h \in L^{\prime} / L, k \in L^{*} / L$, and $r \in L / c L$, we write $h=\left(h_{1}, N h_{2}, 0\right), k=\left(k_{1}, k_{2}, 6 k_{3}\right)$, and $r=\left(N r_{1}, 12 N r_{2}, 6 N r_{3}\right)$. Then the sum on $r$ in (5.9) equals

$$
e\left(\frac{a N h_{2}^{2}-2 h_{2} k_{2}}{24 c}\right) G\left(6 a N, a N h_{2}-k_{2}, c\right) S(c),
$$


where (recalling that $N \mid c$ ),

$$
\begin{aligned}
S(c) & =e\left(\frac{h_{1} k_{3}}{N c}\right) \sum_{r_{1}, r_{3}(c)} e\left(\frac{-a N r_{1} r_{3}-a h_{1} r_{3}+r_{1} k_{3}+r_{3} k_{1}}{c}\right) \\
& = \begin{cases}N c e\left(\frac{d k_{1} k_{3}^{\prime}}{c}\right) & \text { if } k_{3}=N k_{3}^{\prime} \text { and } k_{1} \equiv a h_{1} \quad(\bmod N), \\
0 & \text { otherwise. }\end{cases}
\end{aligned}
$$

So by (5.10), (5.11), and (15.6) we have $c(h, k)_{\sigma}=0$ unless $k=\left(k_{1}, N k_{2}^{\prime}, 6 N k_{3}^{\prime}\right) \in L^{\prime}$, in which case $k_{1} \equiv a h_{1}(\bmod N)$. For such $k$ we have

$$
\sum_{h \in L^{\prime} / L} \bar{\psi}_{1}(h) c(h, k)_{\sigma}=\frac{\bar{\psi}\left(d k_{1}\right)}{\sqrt{12 c^{\prime}}} e\left(\frac{d\left(k_{2}^{\prime}\right)^{2}}{24 c^{\prime}}\right) \sum_{h_{2} \bmod 12}\left(\frac{12}{h_{2}}\right) e\left(\frac{a h_{2}^{2}-2 h_{2} k_{2}^{\prime}}{24 c^{\prime}}\right) G\left(6 a, a h_{2}-k_{2}^{\prime}, c^{\prime}\right) .
$$

Applying Lemma 5.5 we have

$$
\sqrt{-i} \sum_{h \in L^{\prime} / L} \bar{\psi}_{1}(h) c(h, k)_{\sigma}=\bar{\psi}(d) \bar{\psi}_{1}(k) \sqrt{-i} e\left(\frac{a+d}{24 c^{\prime}}\right) e^{\pi i s\left(-a, c^{\prime}\right)} .
$$

By (68.4) and (68.5) of [R4] we have $s\left(-a, c^{\prime}\right)=-s\left(a, c^{\prime}\right)=-s\left(d, c^{\prime}\right)$. Therefore

$$
\theta(\sigma z, f)=\bar{\psi}(d) \chi\left(\left(\begin{array}{cc}
a & N b \\
c^{\prime} & d
\end{array}\right)\right)(c z+d)^{\frac{1}{2}} \theta(z, f) .
$$

By (2.9) and the assumption $N \equiv 1(\bmod 24)$, we have $\chi\left(\left(\begin{array}{cc}a & N b \\ c^{\prime} & d\end{array}\right)\right)=\left(\frac{N}{d}\right) \chi\left(\left(\begin{array}{ll}a & b \\ c & d\end{array}\right)\right)$, from which the lemma follows.

For $w=\xi+i \eta \in \mathbb{H}$ and $0 \leq \theta<2 \pi$, let

$$
g=g(w, \theta)=\left(\begin{array}{ll}
1 & \xi \\
0 & 1
\end{array}\right)\left(\begin{array}{cc}
\eta^{\frac{1}{2}} & 0 \\
0 & \eta^{-\frac{1}{2}}
\end{array}\right)\left(\begin{array}{cc}
\cos \theta & \sin \theta \\
-\sin \theta & \cos \theta
\end{array}\right) \in \mathrm{SL}_{2}(\mathbb{R}) .
$$

The action of $\mathrm{SL}_{2}(\mathbb{R})$ on $\mathbb{R}^{3}$ is given by $g \cdot x=x^{\prime}$, where

$$
\left(\begin{array}{cc}
x_{1}^{\prime} & x_{2}^{\prime} / 2 \\
x_{2}^{\prime} / 2 & x_{3}^{\prime}
\end{array}\right)=g\left(\begin{array}{cc}
x_{1} & x_{2} / 2 \\
x_{2} / 2 & x_{3}
\end{array}\right) g^{T}
$$

Since $\langle x, x\rangle=-\frac{1}{3 N} \operatorname{det}\left(\begin{array}{cc}x_{1} & x_{2} / 2 \\ x_{2} / 2 & x_{3}\end{array}\right)$ we have $\langle g x, g x\rangle=\langle x, x\rangle$. The action of $\mathrm{SL}_{2}(\mathbb{R})$ on functions $f: \mathbb{R}^{3} \rightarrow \mathbb{C}$ is given by $g f(x):=f\left(g^{-1} x\right)$.

We specialize to $f=f_{3}$, with

$$
f_{3}(x)=\exp \left(-\frac{\pi}{12 N}\left(2 x_{1}^{2}+x_{2}^{2}+2 x_{3}^{2}\right)\right)
$$

as in [N, Example 3], and we consider the function $\theta\left(z, g f_{3}\right)$. Let $k(\theta)=\left(\begin{array}{c}\cos \theta \sin \theta \\ -\sin \theta \cos \theta\end{array}\right)$. Since $f_{3}(k(\theta) x)=f_{3}(x)$, the function $\theta\left(z, g f_{3}\right)$ is independent of the variable $\theta$. Therefore it makes sense to define

$$
\vartheta(z, w):=\theta\left(z, g(w, 0) f_{3}\right)=v^{\frac{1}{2}} \sum_{x \in L^{\prime}} \bar{\psi}_{1}(x) e\left(\frac{u}{2}\langle x, x\rangle\right) f_{3}\left(\sqrt{v} \sigma_{w}^{-1} x\right),
$$

where the second equality follows from

$$
\left[r_{0}\left(\sigma_{z}\right) f\right](x)=v^{\frac{3}{4}} e\left(\frac{u}{2}\langle x, x\rangle\right) f(\sqrt{v} x) .
$$


To determine the transformation of $\vartheta(z, w)$ in the variable $w$, we use the relation

$$
\sigma_{\gamma w}=\gamma \sigma_{w} k(\arg (c w+d)), \quad \gamma=\left(\begin{array}{ll}
a & b \\
c & d
\end{array}\right) \in \mathrm{SL}_{2}(\mathbb{R}) .
$$

Suppose that $\gamma=\left(\begin{array}{ll}a & b \\ c & d\end{array}\right) \in \Gamma_{0}(6 N)$. If $x^{\prime}=\gamma x$ then by (5.12) we have

$$
\begin{aligned}
& x_{1}^{\prime}=a^{2} x_{1}+a b x_{2}+b^{2} x_{3}, \\
& x_{2}^{\prime}=2 a c x_{1}+(1+2 b c) x_{2}+2 b d x_{3}, \\
& x_{3}^{\prime}=c^{2} x_{1}+c d x_{2}+d^{2} x_{3} .
\end{aligned}
$$

It follows that $\gamma L^{\prime}=L^{\prime}$ and that $\psi_{1}\left(x^{\prime}\right)=\psi^{2}(a) \psi_{1}(x)$. Thus

$$
\vartheta(z, \gamma w)=\psi^{2}(d) \vartheta(z, w) \quad \text { for all } \gamma \in \Gamma_{0}(6 N) .
$$

A computation shows that replacing $w=\xi+i \eta$ by $w^{\prime}=-\xi+i \eta$ in $f_{3}\left(\sqrt{v} \sigma_{w}^{-1} x\right)$ has the same effect as replacing $x_{2}$ by $-x_{2}$, so

$$
\vartheta(z,-\xi+i \eta)=\vartheta(z, \xi+i \eta) .
$$

5.2. Proof of Theorem 5.1 in the case $t=1$. Suppose that $F \in \mathcal{S}_{\frac{1}{2}}\left(N, \bar{\psi}\left(\frac{N}{\bullet}\right) \chi, r\right)$ with $r \neq i / 4$. For $z=u+i v$ and $w=\xi+i \eta$ we define

$$
\Psi_{F}(w):=\int_{\mathcal{D}} v^{\frac{1}{4}} \overline{\vartheta(z, w)} F(z) d \mu,
$$

where $\mathcal{D}=\Gamma_{0}(N) \backslash \mathbb{H}$ and $d \mu=\frac{d u d v}{v^{2}}$. Note that the integral is well-defined by Lemma 5.4. There is no issue with convergence since $\vartheta$ and $F$ are both rapidly decreasing at the cusps.

Let $D^{(w)}$ denote the Casimir operator for $\mathrm{SL}_{2}(\mathbb{R})$ defined by

$$
D^{(w)}=\eta^{2}\left(\frac{\partial^{2}}{\partial \xi^{2}}+\frac{\partial^{2}}{\partial \eta^{2}}\right)-\eta \frac{\partial^{2}}{\partial \xi \partial \theta} .
$$

To show that $\Psi_{F}$ is an eigenform of $\Delta_{0}^{(w)}$, we use Lemmas 1.4 and 1.5 of [Sh], which give

$$
v^{-\frac{1}{2}} r_{0}\left(\sigma_{z}\right) D^{(w)} f=\Delta_{\frac{1}{2}}^{(z)}\left[v^{-\frac{1}{2}} r_{0}\left(\sigma_{z}\right) f\right] .
$$

Since $\Psi_{F}$ is constant with respect to $\theta$, we have $\Delta_{0}^{(w)} \Psi_{F}=D^{(w)} \Psi_{F}$. By the lemma on p. 304 of $[\mathrm{Sa}$, it follows that

$$
\Delta_{0}^{(w)} \Psi_{F}+\left(\frac{1}{4}+(2 r)^{2}\right) \Psi_{F}=0 .
$$

This, together with the transformation law (5.16) shows that $\Psi_{F}$ is a Maass form. The following lemma shows that $\Psi_{F}$ is a cusp form. This is the only point where we use the assumption that $N$ is squarefree. It would be possible to remove this assumption with added complications by arguing as in [C]. For the remainder of this section, to avoid confusion with the eta function, we write $w=\xi+i y$.

Lemma 5.6. Suppose that $N \equiv 1(\bmod 24)$ is squarefree and that $F \in \mathcal{S}_{\frac{1}{2}}\left(N, \bar{\psi}\left(\frac{N}{\bullet}\right) \chi, r\right)$. Let $\eta_{N}(w):=y^{\frac{1}{4}} \eta(N w)$. For each cusp $\mathfrak{a}=\gamma_{\mathfrak{a}} \infty$ there exists $c_{\mathfrak{a}} \in \mathbb{C}$ such that as $y \rightarrow \infty$ we have

$$
\left(\left.\Psi_{F}\right|_{0} \gamma_{\mathfrak{a}}\right)(i y)= \begin{cases}c_{\mathfrak{a}}\left\langle\eta_{N}, F\right\rangle y+O(1) & \text { if } \psi \text { is principal }, \\ O(1) & \text { otherwise. }\end{cases}
$$


Proof. For each $d \mid 6 N$, let $\left.f \mapsto f\right|_{0} W_{d}$ be the Atkin-Lehner involution [AL, §2] given by any matrix $W_{d} \in \mathrm{SL}_{2}(\mathbb{R})$ of the form

$$
W_{d}=\left(\begin{array}{cc}
\sqrt{d} \alpha & \beta / \sqrt{d} \\
6 N \gamma / \sqrt{d} & \sqrt{d} \delta
\end{array}\right), \quad \alpha, \beta, \gamma, \delta \in \mathbb{Z} .
$$

Since $N \equiv 1(\bmod 24)$ is squarefree, every cusp of $\Gamma_{0}(6 N)$ is of the form $W_{d} \infty$ for some $d$. Thus it suffices to establish (5.18) for $\gamma_{\mathfrak{a}}=W_{d}$.

By (5.14) - (5.15) we have $W_{d} L^{\prime}=L^{\prime}$, so

$$
\vartheta_{d}(z, w):=\vartheta\left(z, W_{d} w\right)=v^{\frac{1}{2}} \sum_{x \in L^{\prime}} \bar{\psi}_{1}\left(W_{d} x\right) e\left(\frac{u}{2}\langle x, x\rangle\right) f_{3}\left(\sqrt{v} \sigma_{w}^{-1} x\right) .
$$

To determine the asymptotic behavior of $\vartheta_{d}(z, i y)$ as $y \rightarrow \infty$, we follow the method of Cipra [C, $§ 4.3]$. We write

$$
\vartheta_{d}(z, i y)=v^{\frac{1}{2}} \sum_{\substack{x \in L^{\prime} \\ x_{3}=0}} \bar{\psi}_{1}\left(W_{d} x\right) e\left(\frac{z}{24 N} x_{2}^{2}\right) \exp \left(-\frac{\pi v}{6 N y^{2}} x_{1}^{2}\right)+\varepsilon(z, y),
$$

where

$$
|\varepsilon(z, y)| \leq v^{\frac{1}{2}} \sum_{x_{1} \in \mathbb{Z}} \exp \left(\frac{-\pi v}{6 N y^{2}} x_{1}^{2}\right) \sum_{x_{2} \in \mathbb{Z}} \exp \left(\frac{-\pi v}{12 N} x_{2}^{2}\right) \sum_{x_{3} \neq 0} \exp \left(\frac{-\pi v y^{2}}{6 N} x_{3}^{2}\right) \ll\left(v+\frac{1}{v}\right) y e^{-c_{1} v y^{2}}
$$

for some $c_{1}>0$ (see [C, Appendix B]). As in [C, Proposition B.3], the contribution of $\varepsilon(z, y)$ to $\left(\left.\Psi_{F}\right|_{0} W_{d}\right)(i y)$ is $o(1)$ as $y \rightarrow \infty$. For $x_{3}=0$ and $W_{d} x=\left(x_{1}^{\prime}, x_{2}^{\prime}, x_{3}^{\prime}\right)$ we have

$$
\begin{aligned}
x_{1}^{\prime} & \equiv d \alpha^{2} x_{1} \quad(\bmod N), \\
x_{2}^{\prime} & \equiv\left(1+\frac{12 N}{d} \gamma \beta\right) x_{2} \quad(\bmod 12)
\end{aligned}
$$

(in particular, $\bar{\psi}_{1}\left(W_{d} x\right)=0$ unless $d \in\{1,2,3,6\}$ ). Thus for some $c_{2}$ the main term of (5.19) equals

$$
\begin{aligned}
c_{2} v^{\frac{1}{2}} \sum_{x_{1} \in \mathbb{Z}} \bar{\psi}\left(x_{1}\right) \exp \left(-\frac{\pi v}{6 N y^{2}} x_{1}^{2}\right) \sum_{x_{2} \in N \mathbb{Z}}\left(\frac{12}{x_{2}}\right) & e\left(\frac{z}{24 N} x_{2}^{2}\right) \\
& =2 c_{2} \eta(N z) v^{\frac{1}{2}} \sum_{h \bmod N} \bar{\psi}(h) \theta_{1}\left(\frac{i v}{12 N y^{2}}, h, N\right),
\end{aligned}
$$

where $\theta_{1}(\cdot, h, N)$ is as in [C, Theorem 1.10(i)]. By the first two assertions of that theorem we have

$$
v^{\frac{1}{2}} \sum_{h \bmod N} \bar{\psi}(h) \theta_{1}\left(\frac{i v}{12 N y^{2}}, h, N\right)=\sqrt{\frac{6}{N}} y \sum_{h \bmod N} \bar{\psi}(h)+O_{N}(\sqrt{v}) .
$$

The latter sum is zero unless $\psi$ is principal. In that case we have

$$
\left(\left.\Psi_{F}\right|_{0} W_{d}\right)(i y)=c_{3} y \int_{\mathcal{D}} v^{\frac{1}{4}} \overline{\eta(N z)} F(z) d \mu+O(1)
$$

for some $c_{3} \in \mathbb{C}$; otherwise $\Psi_{F}$ is $O(1)$ at the cusps.

If the spectral parameter of $F$ is not $i / 4$ then $F$ is orthogonal to $\eta_{N}$. Since a Maass form that is bounded at the cusps is a cusp form, we obtain the following proposition (recall (5.17)). 
Proposition 5.7. Suppose that $F \in \mathcal{S}_{\frac{1}{2}}\left(N, \bar{\psi}\left(\frac{N}{\bullet}\right) \chi, r\right)$, where $N \equiv 1(\bmod 24)$ is squarefree and $r \neq i / 4$. Then $\Psi_{F}$ is an even Maass cusp form in $\mathcal{S}_{0}\left(6 N, \bar{\psi}^{2}, 2 r\right)$.

With $G$ as in Theorem 5.1 let $F:=\left.G\right|_{\frac{1}{2}} w_{N}$, where

$$
w_{N}=\left(\begin{array}{cc}
0 & -1 / \sqrt{N} \\
\sqrt{N} & 0
\end{array}\right)
$$

is the Fricke involution. To obtain the $t=1$ case of Theorem 5.1 we will compute the Fourier expansion of

$$
\Phi_{F}(w):=\Psi_{F}(-1 / 6 N w) .
$$

Once we show that the coefficients of $\Phi_{F}$ satisfy the relation (5.2), Proposition 5.7completes the proof. We first show that $F$ defined in this way satisfies the hypotheses of Proposition 5.7.

Lemma 5.8. Suppose that $N \equiv 1(\bmod 24)$. If $G \in \mathcal{S}_{\frac{1}{2}}(N, \psi \chi, r)$ and $F=\left.G\right|_{\frac{1}{2}} w_{N}$, then $F \in \mathcal{S}_{\frac{1}{2}}\left(N, \bar{\psi}\left(\frac{N}{\bullet}\right) \chi, r\right)$.

Proof. Since the slash operator commutes with $\Delta_{k}$, it suffices to show that $F$ transforms with mutliplier $\bar{\psi}\left(\frac{N}{\bullet}\right) \chi$. Writing $\gamma=\left(\begin{array}{ll}a & b \\ c & d\end{array}\right) \in \Gamma_{0}(N)$ and $\gamma^{\prime}=w_{N} \gamma w_{N}^{-1}=\left(\begin{array}{cc}d & -c / N \\ -b N & a\end{array}\right)$ we have

$$
\left.F\right|_{\frac{1}{2}} \gamma=\bar{\psi}(d) \chi\left(\gamma^{\prime}\right) F \text {. }
$$

Thus it suffices to show that $\chi\left(\gamma^{\prime}\right)=\left(\frac{N}{d}\right) \chi(\gamma)$. For this we argue as in [ㅍ, Proposition 1.4].

Write $w_{N}=S V$, where

$$
S=\left(\begin{array}{cc}
0 & -1 \\
1 & 0
\end{array}\right), \quad V=\left(\begin{array}{cc}
\sqrt{N} & 0 \\
0 & 1 / \sqrt{N}
\end{array}\right) .
$$

Let $\widetilde{\eta}(w):=y^{1 / 4} \eta(w)$ and let $g:=\left.\widetilde{\eta}\right|_{\frac{1}{2}} V$. Then by (2.9), $g$ transforms on $\Gamma_{0}(N)$ with multiplier $\left(\frac{N}{\bullet}\right) \chi$. We compute

$$
\chi\left(w_{N} \gamma w_{N}^{-1}\right) \widetilde{\eta}=\left.\widetilde{\eta}\right|_{\frac{1}{2}} w_{N} \gamma w_{N}^{-1}=\left.\sqrt{-i} g\right|_{\frac{1}{2}} \gamma w_{N}^{-1}=\left.\sqrt{-i}\left(\frac{N}{d}\right) \chi(\gamma) g\right|_{\frac{1}{2}} w_{N}^{-1}=\left(\frac{N}{d}\right) \chi(\gamma) \widetilde{\eta} .
$$

We will determine the Fourier coefficients of $\Phi_{F}$ by computing its Mellin transform $\Omega(s)$ in two ways. We have

$$
\Omega(s):=\int_{0}^{\infty} \Phi_{F}(i y) y^{s} \frac{d y}{y}=(6 N)^{-s} \int_{0}^{\infty} \Psi_{F}(i / y) y^{s} \frac{d y}{y} .
$$

For $w$ purely imaginary, the sum in (5.13) simplifies to

$$
\vartheta(z, i y)=\vartheta_{1}(z) \vartheta_{2}(z, y),
$$

where $\vartheta_{1}(z)=2 \eta(N z)$ and

$$
\begin{aligned}
\vartheta_{2}(z, y) & =v^{\frac{1}{2}} \sum_{m, n \in \mathbb{Z}} \bar{\psi}(m) e(-u m n) \exp \left(-\pi v\left(\frac{m^{2}}{6 N y^{2}}+6 N y^{2} n^{2}\right)\right) \\
& =\frac{1}{y \sqrt{6 N}} \sum_{m, n \in \mathbb{Z}} \bar{\psi}(m) \exp \left(-\frac{\pi}{6 N y^{2} v}|m z+n|^{2}\right) .
\end{aligned}
$$


The latter equality follows from Poisson summation on $n$.

Setting $A:=\frac{\pi}{6 N v}|m z+n|^{2}$ for the moment, we find that

$$
\Omega(s)=2(6 N)^{-s-\frac{1}{2}} \sum_{m, n} \psi(m) \int_{\mathcal{D}} v^{\frac{1}{4}} \overline{\eta(N z)} F(z) \int_{0}^{\infty} y^{s} e^{-A y^{2}} d y d \mu .
$$

For $\operatorname{Re}(s)>1$ the inner integral evaluates to $\frac{1}{2} A^{\frac{-s-1}{2}} \Gamma\left(\frac{s+1}{2}\right)$, so we have

$$
\Omega(s)=\frac{\Gamma\left(\frac{s+1}{2}\right)}{(6 N)^{\frac{s}{2}} \pi^{\frac{s+1}{2}}} \sum_{m, n} \psi(m) \int_{\mathcal{D}} v^{\frac{1}{4}} \overline{\eta(N z)} F(z)\left(\frac{v}{|m z+n|^{2}}\right)^{\frac{s+1}{2}} d \mu .
$$

Replacing $z$ by $w_{N} z$, recalling that $G=\left.F\right|_{\frac{1}{2}} w_{N}$ and using the fact that $\psi$ is even, we obtain

$$
\Omega(s)=\frac{\sqrt{i} N^{\frac{1}{4}}}{6^{\frac{s}{2}} \pi^{\frac{s+1}{2}}} \Gamma\left(\frac{s+1}{2}\right) \sum_{m, n} \psi(m) \int_{\mathcal{D}} v^{\frac{1}{4}} \overline{\eta(z)} G(z)\left(\frac{v}{|N n z+m|^{2}}\right)^{\frac{s+1}{2}} d \mu .
$$

For $(m, N)=1$, write $m=g d$ and $N n=g c$ with $g=(m, n)=(m, N n)$ so that

$$
\sum_{m, n} \psi(m)\left(\frac{v}{|N n z+m|^{2}}\right)^{\frac{s+1}{2}}=L(s+1, \psi) \sum_{\gamma \in \Gamma_{\infty} \backslash \Gamma_{0}(N)} \psi(\gamma) \operatorname{Im}(\gamma z)^{\frac{s+1}{2}},
$$

where $\psi(\gamma)=\psi(d)$ for $\gamma=\left(\begin{array}{ll}a & b \\ c & d\end{array}\right)$. Thus

$$
\Omega(s)=c(s) \sum_{\gamma \in \Gamma_{\infty} \backslash \Gamma_{0}(N)} \psi(\gamma) \int_{\mathcal{D}} v^{\frac{1}{4}} \overline{\eta(z)} G(z) \operatorname{Im}(\gamma z)^{\frac{s+1}{2}} d \mu,
$$

where

$$
c(s)=\frac{\sqrt{i} N^{\frac{1}{4}}}{6^{\frac{s}{2}} \pi^{\frac{s+1}{2}}} \Gamma\left(\frac{s+1}{2}\right) L(s+1, \psi) .
$$

Since $\psi(\gamma) v^{1 / 4} \overline{\eta(z)} G(z)=\operatorname{Im}(\gamma z)^{1 / 4} \overline{\eta(\gamma z)} G(\gamma z)$ for $\gamma \in \Gamma$, we have

$$
\Omega(s)=c(s) \int_{\Gamma_{\infty} \backslash \mathbb{H}} v^{\frac{s+1}{2}+\frac{1}{4}} \overline{\eta(z)} G(z) d \mu .
$$

Recall that $G$ has Fourier expansion

$$
G(z)=\sum_{n \equiv 1(24)} a(n) W_{\frac{\operatorname{sgn}(n)}{4}, i r}\left(\frac{\pi|n| v}{6}\right) e\left(\frac{n u}{24}\right) .
$$

Thus we have

$$
\Omega(s)=c(s) \sum_{n=1}^{\infty}\left(\frac{12}{n}\right) a\left(n^{2}\right) \int_{0}^{\infty} v^{\frac{s}{2}-\frac{5}{4}} W_{\frac{1}{4}, i r}\left(\frac{\pi n^{2} v}{6}\right) e^{-\frac{\pi n^{2}}{12} v} d v .
$$

By [DL, (13.23.4) and (16.2.5)], the integral evaluates to

$$
\left(\frac{6}{\pi n^{2}}\right)^{\frac{s}{2}-\frac{1}{4}} \frac{\Gamma\left(\frac{s}{2}+\frac{1}{4}+i r\right) \Gamma\left(\frac{s}{2}+\frac{1}{4}-i r\right)}{\Gamma\left(\frac{s+1}{2}\right)}
$$

so we conclude that

$$
\Omega(s)=\sqrt{i}\left(\frac{\pi N}{6}\right)^{\frac{1}{4}} \pi^{-s-\frac{1}{2}} \Gamma\left(\frac{s}{2}+\frac{1}{4}+i r\right) \Gamma\left(\frac{s}{2}+\frac{1}{4}-i r\right) L(s+1, \psi) \sum_{n=1}^{\infty}\left(\frac{12}{n}\right) \frac{a\left(n^{2}\right)}{n^{s-\frac{1}{2}}} .
$$


On the other hand, since $\Psi_{F}$ is an even Maass cusp form, it follows that $\Phi_{F}$ is also even. Since $\Phi_{F}$ has eigenvalue $\frac{1}{4}+(2 r)^{2}$, it has a Fourier expansion of the form

$$
\Phi_{F}(w)=2 \sum_{n=1}^{\infty} b(n) W_{0,2 i r}(4 \pi n y) \cos (2 \pi n x) .
$$

By the definition (5.20) of $\Omega(s)$ and [DL, (13.18.9) and (10.43.19)] we have

$$
\begin{aligned}
\Omega(s) & =4 \sum_{n=1}^{\infty} \sqrt{n} b(n) \int_{0}^{\infty} K_{2 i r}(2 \pi n y) y^{s-\frac{1}{2}} d y \\
& =\pi^{-s-\frac{1}{2}} \Gamma\left(\frac{s}{2}+\frac{1}{4}+i r\right) \Gamma\left(\frac{s}{2}+\frac{1}{4}-i r\right) \sum_{n=1}^{\infty} \frac{b(n)}{n^{s}} .
\end{aligned}
$$

So the coefficients $b(n)$ are given by the relation

$$
\sum_{n=1}^{\infty} \frac{b(n)}{n^{s}}=\sqrt{i}\left(\frac{\pi N}{6}\right)^{\frac{1}{4}} L(s+1, \psi) \sum_{n=1}^{\infty}\left(\frac{12}{n}\right) \frac{a\left(n^{2}\right)}{n^{s-\frac{1}{2}}} .
$$

This proves Theorem 5.1 in the case $t=1$ since $b(n)$ is a constant multiple of the function $b_{1}(n)$ in (5.2).

5.3. The case $t>1$. For squarefree $t \equiv 1(\bmod 24)$ with $t>1$ we argue as in Section 3 of [N]. For a function $f$ on $\mathbb{H}$ define $f_{t}(\tau):=f(t \tau)$. We apply Theorem 5.1 to

$$
G_{t}(\tau)=\sum_{n \equiv 1(24)} a(n / t) W_{\frac{\operatorname{sgn}(n)}{4}, i r}\left(\frac{\pi|n| y}{6}\right) e\left(\frac{n x}{24}\right) \in \mathcal{S}_{\frac{1}{2}}\left(N t, \psi\left(\frac{t}{\bullet}\right) \chi, r\right) .
$$

The coefficients $c(n)$ of $S_{1}\left(G_{t}\right) \in \mathcal{S}_{0}\left(6 N t, \psi^{2}, 2 r\right)$ are given by

$$
\sum_{n=1}^{\infty} \frac{c(n)}{n^{s}}=L\left(s+1, \psi\left(\frac{t}{\bullet}\right)\right) \sum_{n=1}^{\infty}\left(\frac{12}{n}\right) \frac{a\left(n^{2} / t\right)}{n^{s-\frac{1}{2}}}=L\left(s+1, \psi\left(\frac{t}{\bullet}\right)\right) t^{-s+\frac{1}{2}} \sum_{n=1}^{\infty}\left(\frac{12}{n}\right) \frac{a\left(t n^{2}\right)}{n^{s-\frac{1}{2}}} .
$$

Thus $c(n)=0$ unless $t \mid n$, in which case $c(n)=\sqrt{t} b_{t}(n / t)$, where $b_{t}(n)$ are the coefficients of $S_{t}(G)$. We conclude that $S_{1}\left(G_{t}\right)=\sqrt{t}\left[S_{t}(G)\right]_{t}$. By a standard argument (see e.g. [N], Section 3]) we have

$$
S_{t}(G) \in \mathcal{S}_{0}\left(6 N, \psi^{2}, 2 r\right)
$$

This completes the proof of Theorem 5.1 .

\section{Estimates for a $K$-Bessel transform}

This section contains uniform estimates for the $K$-Bessel transform $\check{\phi}(r)$ which are required in Sections 7 and 9, Recall that

$$
\check{\phi}(r)=\operatorname{ch} \pi r \int_{0}^{\infty} K_{2 i r}(u) \phi(u) \frac{d u}{u},
$$

where $\phi$ is a suitable test function (see (4.1)). Given $a, x, T>0$ with

$$
T \leq \frac{x}{3} \quad \text { and } \quad T \asymp x^{1-\delta}, \quad 0<\delta<\frac{1}{2},
$$

we choose $\phi=\phi_{a, x, T}:[0, \infty) \rightarrow[0,1]$ to be a smooth function satisfying

(i) $\phi(t)=1$ for $\frac{a}{2 x} \leq t \leq \frac{a}{x}$, 
(ii) $\phi(t)=0$ for $t \leq \frac{a}{2 x+2 T}$ and $t \geq \frac{a}{x-T}$,

(iii) $\phi^{\prime}(t) \ll\left(\frac{a}{x-T}-\frac{a}{x}\right)^{-1} \ll \frac{x^{2}}{a T}$, and

(iv) $\phi$ and $\phi^{\prime}$ are piecewise monotone on a fixed number of intervals (whose number is independent of $a, x$, and $T$ ).

In Theorem 4.1, the function $\check{\phi}(r)$ is evaluated at the spectral parameters $r_{j}$ corresponding to the eigenfunctions $u_{j}$ as in (2.23). In view of Corollary 5.3, we require estimates only for $r \geq 1$. We will prove the following theorem.

Theorem 6.1. Suppose that $a, x, T$, and $\phi=\phi_{a, x, T}$ are as above. Then

$$
\check{\phi}(r) \ll \begin{cases}r^{-\frac{3}{2}} e^{-r / 2} & \text { if } 1 \leq r \leq \frac{a}{8 x}, \\ r^{-1} & \text { if } \max \left(\frac{a}{8 x}, 1\right) \leq r \leq \frac{a}{x}, \\ \min \left(r^{-\frac{3}{2}}, r^{-\frac{5}{2}} \frac{x}{T}\right) & \text { if } r \geq \max \left(\frac{a}{x}, 1\right) .\end{cases}
$$

To prove Theorem 6.1 we require estimates for $K_{i v}(v z)$ which are uniform for $z \in(0, \infty)$ and $v \in[1, \infty)$.

6.1. Uniform estimates for the $K$-Bessel function. We estimate $K_{i v}(v z)$ in the following ranges as $v \rightarrow \infty$ :

(A) the oscillatory range $0<z \leq 1-O\left(v^{-\frac{2}{3}}\right)$,

(B) the transitional range $1-O\left(v^{-\frac{2}{3}}\right) \leq z \leq 1+O\left(v^{-\frac{2}{3}}\right)$, and

(C) the decaying range $z \geq 1+O\left(v^{-\frac{2}{3}}\right)$.

Suppose that $c$ is a positive constant. In the transitional range there is a significant "bump" in the $K$-Bessel function. By [BST, (14) and (21)] we have

$$
e^{\frac{\pi v}{2}} K_{i v}(v z) \ll_{c} v^{-\frac{1}{3}} \quad \text { for } z \geq 1-c v^{-\frac{2}{3}} .
$$

In the decaying range the $K$-Bessel function is positive and decreasing. By [BST, (14)] we have

$$
e^{\frac{\pi v}{2}} K_{i v}(v z) \ll_{c} \frac{e^{-v \mu(z)}}{v^{\frac{1}{2}}\left(z^{2}-1\right)^{\frac{1}{4}}} \quad \text { for } z \geq 1+c v^{-\frac{2}{3}}
$$

where

$$
\mu(z):=\sqrt{z^{2}-1}-\arccos \left(\frac{1}{z}\right) .
$$

The oscillatory range is much more delicate. Balogh $\mathrm{Ba}$ gives a uniform asymptotic expansion for $K_{i v}(v z)$ in terms of the Airy function Ai and its derivative $\mathrm{Ai}^{\prime}$. For $z \in(0,1)$ define

$$
w(z):=\operatorname{arccosh}\left(\frac{1}{z}\right)-\sqrt{1-z^{2}}
$$

and define $\zeta$ and $\xi$ by

$$
\frac{2}{3} \zeta^{\frac{3}{2}}=-i w(z), \quad \xi=v^{\frac{2}{3}} \zeta
$$


Taking $m=1$ in equation (2) of [Ba] we have

$$
e^{\frac{\pi v}{2}} K_{i v}(v z)=\frac{\pi \sqrt{2}}{v^{\frac{1}{3}}}\left(\frac{-\zeta}{1-z^{2}}\right)^{\frac{1}{4}}\left\{\operatorname{Ai}(\xi)\left[1+\frac{A_{1}(\zeta)}{v^{2}}\right]+\mathrm{Ai}^{\prime}(\xi) \frac{B_{0}(\zeta)}{v^{\frac{4}{3}}}+\frac{e^{i v w(z)}}{1+|\xi|^{\frac{1}{4}}} O\left(v^{-3}\right)\right\},
$$

uniformly for $v \in[1, \infty)$, where

$$
\begin{aligned}
& A_{1}(\zeta):=\frac{455}{10368 w(z)^{2}}-\frac{7\left(3 z^{2}+2\right)}{1728\left(1-z^{2}\right)^{\frac{3}{2}} w(z)}-\frac{81 z^{4}+300 z^{2}+4}{1152\left(1-z^{2}\right)^{3}}, \\
& B_{0}(\zeta):=\left(\frac{2}{3}\right)^{\frac{1}{3}} \frac{e^{2 \pi i / 3}}{w(z)^{\frac{1}{3}}}\left(\frac{3 z^{2}+2}{24\left(1-z^{2}\right)^{\frac{3}{2}}}-\frac{5}{72 w(z)}\right) .
\end{aligned}
$$

A computation shows that $A_{1}(\zeta)$ and $B_{0}(\zeta)$ have finite limits as $z \rightarrow 0^{+}$and as $z \rightarrow 1^{-}$, so both functions are $O(1)$ for $z \in(0,1)$.

Note that $\arg \xi=-\frac{\pi}{3}$. In order to work on the real line, we apply [DL, (9.6.2-3)] to obtain

$$
\begin{aligned}
\operatorname{Ai}(\xi) & =\left(\frac{2}{3}\right)^{1 / 6} \frac{i}{2^{3 / 2}}(v w(z))^{\frac{1}{3}} H_{\frac{1}{3}}^{(1)}(v w(z)), \\
\operatorname{Ai}^{\prime}(\xi) & =-\left(\frac{3}{2}\right)^{1 / 6} \frac{i}{2^{3 / 2}}(v w(z))^{\frac{2}{3}} H_{\frac{2}{3}}^{(1)}(v w(z)),
\end{aligned}
$$

where $H_{\frac{1}{3}}^{(1)}$ and $H_{\frac{2}{3}}^{(1)}$ are Hankel functions of the first kind. So we have

$$
\begin{aligned}
e^{\frac{\pi v}{2}} K_{i v}(v z)=\frac{\pi}{2} e^{2 \pi i / 3} & \frac{w(z)^{\frac{1}{2}}}{\left(1-z^{2}\right)^{\frac{1}{4}}} H_{\frac{1}{3}}^{(1)}(v w(z))\left[1+\frac{A_{1}(\zeta)}{v^{2}}\right] \\
& +\left(\frac{3}{2}\right)^{1 / 3} \frac{\pi}{2} e^{-\pi i / 3} \frac{w(z)^{\frac{5}{6}}}{\left(1-z^{2}\right)^{\frac{1}{4}}} H_{\frac{2}{3}}^{(1)}(v w(z)) \frac{B_{0}(\zeta)}{v}+O\left(\frac{v^{-\frac{7}{2}}}{\left(1-z^{2}\right)^{\frac{1}{4}}}\right) .
\end{aligned}
$$

Since $w(z) \rightarrow \infty$ as $z \rightarrow 0$ and $\left(1-z^{2}\right)^{-\frac{1}{4}} \rightarrow \infty$ as $z \rightarrow 1$, we derive more convenient expressions for (6.4) for $z$ in the intervals $(0,3 / 4]$ and $\left[3 / 16,1-c v^{-\frac{2}{3}}\right)$.

Proposition 6.2. Suppose that $z \in(0,3 / 4]$ and that $v \geq 1$. Then

$$
e^{\frac{\pi v}{2}} K_{i v}(v z)=e^{\pi i / 4}\left(\frac{\pi}{2}\right)^{\frac{1}{2}} \frac{e^{i v w(z)}}{v^{\frac{1}{2}}\left(1-z^{2}\right)^{\frac{1}{4}}}\left[1-i \frac{3 z^{2}+2}{24 v\left(1-z^{2}\right)^{\frac{3}{2}}}\right]+O\left(v^{-\frac{5}{2}}\right) .
$$

Proof. Since $\left(1-z^{2}\right) \gg 1$ for $z \leq 3 / 4$, the error term in (6.4) is $\ll v^{-\frac{7}{2}}$. By [DL, (10.17.5) and $\S 10.17($ iii)] we have

$$
\begin{aligned}
& H_{\frac{1}{3}}^{(1)}(v w(z))=\left(\frac{2}{\pi}\right)^{\frac{1}{2}} e^{-5 \pi i / 12} \frac{e^{i v w(z)}}{(v w(z))^{\frac{1}{2}}}\left(1-\frac{5 i}{72 v w(z)}\right)+O\left((v w(z))^{-\frac{5}{2}}\right), \\
& H_{\frac{2}{3}}^{(1)}(v w(z))=\left(\frac{2}{\pi}\right)^{\frac{1}{2}} e^{-7 \pi i / 12} \frac{e^{i v w(z)}}{(v w(z))^{\frac{1}{2}}}+O\left((v w(z))^{-\frac{3}{2}}\right) .
\end{aligned}
$$

In particular, this implies that

$$
\frac{w(z)^{\frac{1}{2}}}{\left(1-z^{2}\right)^{\frac{1}{4}}} H_{\frac{1}{3}}^{(1)}(v w(z)) \frac{A_{1}(\zeta)}{v^{2}} \ll v^{-\frac{5}{2}}
$$


so by (6.4) we have

$$
e^{\frac{\pi v}{2}} K_{i v}(v z)=e^{\pi i / 4}\left(\frac{\pi}{2}\right)^{\frac{1}{2}} \frac{e^{i v w(z)}}{v^{\frac{1}{2}}\left(1-z^{2}\right)^{\frac{1}{4}}}\left[1-\frac{5 i}{72 v w(z)}+e^{-7 \pi i / 6}\left(\frac{3}{2}\right)^{\frac{1}{3}} w(z)^{\frac{1}{3}} \frac{B_{0}(\zeta)}{v}\right]+O\left(v^{-\frac{5}{2}}\right) .
$$

Using (6.3), we obtain (6.5).

We require some notation for the next proposition. Let $J_{\nu}(x)$ and $Y_{\nu}(x)$ denote the $J$ and $Y$-Bessel functions, and define

$$
M_{\nu}(x)=\sqrt{J_{\nu}^{2}(x)+Y_{\nu}^{2}(x)} .
$$

Proposition 6.3. Suppose that $c>0$. Suppose that $v \geq 1$ and that $\frac{3}{16} \leq z \leq 1-c v^{-\frac{2}{3}}$. Then

$$
e^{\frac{\pi v}{2}} K_{i v}(v z)=\frac{\pi}{2} e^{2 \pi i / 3} \frac{w(z)^{\frac{1}{2}}}{\left(1-z^{2}\right)^{\frac{1}{4}}} M_{\frac{1}{3}}(v w(z)) e^{i \theta_{\frac{1}{3}}(v w(z))}+O_{c}\left(v^{-4 / 3}\right),
$$

where $\theta_{\frac{1}{3}}(x)$ is a real-valued continuous function satisfying

$$
\theta_{\frac{1}{3}}^{\prime}(x)=\frac{2}{\pi x M_{\frac{1}{3}}^{2}(x)} .
$$

Proof. Since $\left(1-z^{2}\right) \gg_{c} v^{-\frac{2}{3}}$ for $z \leq 1-c v^{-\frac{2}{3}}$, the error term in (6.4) is $\ll_{c} v^{-\frac{10}{3}}$. The modulus and phase of $H_{\alpha}^{(1)}(x)$ are given by [DL, $\left.\S 10.18\right]$

$$
H_{\alpha}^{(1)}(x)=M_{\alpha}(x) e^{i \theta_{\alpha}(x)},
$$

where $M_{\alpha}^{2}(x) \theta_{\alpha}^{\prime}(x)=2 / \pi x$. A straightforward computation shows that $w(z) \gg(1-z)^{\frac{3}{2}}$. It follows that $v w(z) \gg_{c} 1$ for $z \leq 1-c v^{-\frac{2}{3}}$, so by [DL, (10.18.17)] we obtain

$$
M_{\alpha}(v w(z)) \ll_{\alpha, c} \frac{1}{(v w(z))^{\frac{1}{2}}} .
$$

This, together with (6.4) and the fact that $A_{1}(\zeta)$ and $B_{0}(\zeta)$ are $O(1)$, gives (6.6).

6.2. Estimates for $\check{\phi}(r)$. We treat each of the three ranges considered in Theorem 6.1 separately in the following propositions. We will make frequent use of an integral estimate which is an immediate corollary of the second mean value theorem for integrals.

Lemma 6.4. Suppose that $f$ and $g$ are continuous functions on $[a, b]$ and that $g$ is piecewise monotonic on $M$ intervals. Then

$$
\left|\int_{a}^{b} f(x) g(x) d x\right| \leq 2 M \sup _{x \in[a, b]}|g(x)| \sup _{[\alpha, \beta] \subseteq[a, b]}\left|\int_{\alpha}^{\beta} f(x) d x\right| .
$$

Proposition 6.5. With the notation of Theorem 6.1, suppose that $1 \leq r \leq a / 8 x$. Then

$$
\check{\phi}(r) \ll r^{-\frac{3}{2}} e^{-r / 2} .
$$

Proof. Since $r \leq a / 8 x$ and $T \leq x / 3$, we have $\frac{a}{4(x+T) r} \geq \frac{3}{2}$. Taking $c=2^{-1 / 3}$ in (6.2) gives

$$
\check{\phi}(r)=\operatorname{ch} \pi r \int_{\frac{a}{4(x+T) r}}^{\frac{a}{2(x-T) r}} K_{2 i r}(2 r y) \phi(2 r y) \frac{d y}{y} \ll r^{-\frac{1}{2}} \int_{\frac{3}{2}}^{\infty} e^{-2 r \mu(y)} \frac{d y}{y\left(y^{2}-1\right)^{\frac{1}{4}}} .
$$


Since $\mu^{\prime}(y)=\sqrt{y^{2}-1} / y$ we have

$$
\check{\phi}(r) \ll r^{-\frac{3}{2}} \int_{\frac{3}{2}}^{\infty}\left(-e^{-2 r \mu(y)}\right)^{\prime} \frac{d y}{\left(y^{2}-1\right)^{\frac{3}{4}}} \ll r^{-\frac{3}{2}} e^{-2 r \mu(3 / 2)},
$$

which, together with $\mu(3 / 2) \approx .277$ proves the proposition.

Proposition 6.6. With the notation of Theorem 6.1, suppose that $\max (a / 8 x, 1) \leq r \leq a / x$. Then

$$
\check{\phi}(r) \ll r^{-1} \text {. }
$$

Before proving Proposition 6.6, we require a lemma describing the behavior of the function $M_{\frac{1}{3}}(x)$ in Proposition 6.3.

Lemma 6.7. The function $\widetilde{M}(x):=x M_{\frac{1}{3}}^{2}(x)$ is increasing on $[0, \infty)$ with $\lim _{x \rightarrow \infty} \widetilde{M}(x)=\frac{2}{\pi}$.

Proof. We will prove that $w(x):=\sqrt{\widetilde{M}(x)}$ is increasing. From [DL, (10.7.3-4), (10.18.17)] we have

$$
\widetilde{M}(0)=0, \quad 0 \leq \widetilde{M}(x) \leq \frac{2}{\pi}, \quad \text { and } \quad \lim _{x \rightarrow \infty} \widetilde{M}(x)=\frac{2}{\pi} .
$$

It is therefore enough to show that $w^{\prime \prime}(x)<0$ for all $x>0$. In view of the second order differential equation [DL, (10.18.14)] satisfied by $w$ it will suffice to prove that

$$
\widetilde{M}(x)>\frac{12 x}{\pi \sqrt{36 x^{2}+5}} .
$$

The inequality (6.8) can be proved numerically, using the expansions of $\widetilde{M}(x)$ at 0 and $\infty$. By [DL, $\S 10.18($ iii)] we have

$$
\widetilde{M}(x) \geq \frac{2}{\pi}\left(1+\sum_{k=1}^{n} \frac{1 \cdot 3 \cdots(2 k-1)}{2 \cdot 4 \cdots(2 k)} \frac{\left(\frac{4}{9}-1\right)\left(\frac{4}{9}-9\right) \cdots\left(\frac{4}{9}-(2 k-1)^{2}\right)}{(2 x)^{2 k}}\right)
$$

for odd $n \geq 1$. Taking $n=3$ in (6.9), we verify numerically that (6.8) holds for $x>2.34$.

From [DL, (10.2.3)] we have

$$
\widetilde{M}(x)=\frac{4 x}{3}\left(J_{\frac{1}{3}}^{2}(x)-J_{\frac{1}{3}}(x) J_{-\frac{1}{3}}(x)+J_{-\frac{1}{3}}^{2}(x)\right) .
$$

So by [DL, (10.8.3)] it follows that the series for $\widetilde{M}(x)$ at 0 is alternating, and that $\widetilde{M}(x)$ is larger than the truncation of this series after the term with exponent $47 / 3$. Moreover, this truncation is larger than $\frac{12 x}{\pi \sqrt{36 x^{2}+5}}$ for $x<2.45$. The claim (6.8) follows.

Proof of Proposition 6.6. Fix $c=\frac{1}{2}$ and write

$$
\begin{aligned}
\check{\phi}(r) & =\check{\phi}_{1}(r)+\check{\phi}_{2}(r)+\check{\phi}_{3}(r) \\
& =\left(\int_{\frac{a}{4(x+T) r}}^{1-c r^{-\frac{2}{3}}}+\int_{1-c r^{-\frac{2}{3}}}^{1+c r^{-\frac{2}{3}}}+\int_{1+c r^{-\frac{2}{3}}}^{\infty}\right) \operatorname{ch~} \pi r K_{2 i r}(2 r y) \phi(2 r y) \frac{d y}{y} .
\end{aligned}
$$

We will show that $\check{\phi}_{i}(r) \ll r^{-1}$ for $i=1,2,3$. Note that $\frac{a}{4(x+T) r} \geq \frac{3}{16}$. 
By Proposition 6.3 we have

$$
\check{\phi}_{1}(r) \ll\left|\int_{\frac{a}{4(x+T) r}}^{1-c r^{-\frac{2}{3}}} e^{i \theta_{\frac{1}{3}}(2 r w(y))} \frac{M_{\frac{1}{3}}(2 r w(y)) w(y)^{\frac{1}{2}} \phi(2 r y)}{y\left(1-y^{2}\right)^{\frac{1}{4}}} d y\right|+r^{-\frac{4}{3}} \int_{\frac{3}{16}}^{1} \phi(2 r y) \frac{d y}{y} .
$$

The error term is $O\left(r^{-4 / 3}\right)$. Using (6.7) and the fact that $w^{\prime}(y)=-\sqrt{1-y^{2}} / y$, the first term in (6.10) equals

$$
\frac{\pi}{2(2 r)^{\frac{3}{2}}}\left|\int_{\frac{a}{4(x+T) r}}^{1-c r^{-\frac{2}{3}}}\left(e^{i \theta_{\frac{1}{3}}(2 r w(y))}\right)^{\prime} \frac{\left(2 r w(y) M_{\frac{1}{3}}^{2}(2 r w(y))\right)^{\frac{3}{2}}}{\left(1-y^{2}\right)^{\frac{3}{4}}} \phi(2 r y) d y\right| .
$$

Note that $w(y)$ is decreasing, so by Lemma 6.7 the function $2 r w(y) M_{\frac{1}{3}}^{2}(2 r w(y))$ is decreasing. We apply Lemma 6.4 three times; first with the decreasing function

$$
g(y)=\left(2 r w(y) M_{\frac{1}{3}}(2 r w(y))\right)^{\frac{3}{2}} \ll 1,
$$

next with the increasing function

$$
g(y)=\left(1-y^{2}\right)^{-\frac{3}{4}} \ll r^{\frac{1}{2}},
$$

and then with $g(y)=\phi(2 r y)$. We conclude that $\check{\phi}_{1}(r) \ll r^{-1}$.

For $\check{\phi}_{2}(r)$ we apply (6.1) to obtain

$$
\check{\phi}_{2}(r) \ll r^{-\frac{1}{3}} \int_{1-c r^{-\frac{2}{3}}}^{1+c r^{-\frac{2}{3}}} d y \ll r^{-1} .
$$

For $\check{\phi}_{3}(r)$ we argue as in the proof of Proposition 6.5 to obtain

$$
\check{\phi}_{3}(r) \ll r^{-\frac{3}{2}} \int_{1+c r^{-\frac{2}{3}}}^{\infty}\left(-e^{-2 r \mu(y)}\right)^{\prime} \frac{d y}{\left(y^{2}-1\right)^{\frac{3}{4}}} .
$$

For $y \geq 1+c r^{-\frac{2}{3}}$ we have $\left(y^{2}-1\right)^{\frac{3}{4}} \gg r^{-\frac{1}{2}}$, so $\check{\phi}_{3}(r) \ll r^{-1}$.

Proposition 6.8. Suppose that $r \geq \max (a / x, 1)$. Then

$$
\check{\phi}(r) \ll \min \left(r^{-\frac{3}{2}}, r^{-\frac{5}{2}} \frac{x}{T}\right) .
$$

Proof. Since $r \geq a / x$ and $T \leq x / 3$, the interval on which $\phi(2 r y) \neq 0$ is contained in $(0,3 / 4]$. So by Proposition 6.2 we have

$$
\check{\phi}(r) \ll r^{-\frac{1}{2}}\left|I_{0}\right|+r^{-\frac{3}{2}}\left|I_{1}\right|+r^{-\frac{5}{2}} \int_{\frac{a}{4(x+T) r}}^{\frac{a}{2(x-T) r}} \frac{d y}{y},
$$

where

$$
I_{\alpha}=\int_{\frac{a}{4(x+T) r}}^{\frac{a}{2(x-T) r}} e^{2 i r w(y)} \frac{\phi(2 r y)\left(3 y^{2}+2\right)^{\alpha}}{y\left(1-y^{2}\right)^{\frac{1}{4}+\frac{3}{2} \alpha}} d y .
$$

The third term in (6.11) equals

$$
r^{-\frac{5}{2}} \log \frac{2(x+T)}{x-T} \ll r^{-\frac{5}{2}}
$$


For the other terms we use $w^{\prime}(y)=-\sqrt{1-y^{2}} / y$ to write

$$
I_{\alpha} \ll r^{-1}\left|\int_{\frac{a}{4(x+T) r}}^{\frac{a}{2(x-T) r}}\left(e^{2 i r w(y)}\right)^{\prime} \frac{\phi(2 r y)\left(3 y^{2}+2\right)^{\alpha}}{\left(1-y^{2}\right)^{\frac{3}{4}+\frac{3}{2} \alpha}} d y\right| .
$$

Since $y \leq 3 / 4$ we have

$$
\frac{\left(3 y^{2}+2\right)^{\alpha}}{\left(1-y^{2}\right)^{\frac{3}{4}+\frac{3}{2} \alpha}} \ll 1
$$

so by Lemma 6.4 we obtain $I_{\alpha} \ll r^{-1}$ and therefore $\check{\phi}(r) \ll r^{-\frac{3}{2}}$.

For large $r$, we obtain a better estimate by integrating by parts in (6.12) for $\alpha=0$. Since $\phi(2 r y)=0$ at the limits of integration, we find that

$$
I_{0} \ll r^{-1}\left|\int_{\frac{a}{4(x+T) r}}^{\frac{a}{2(x-T) r}} e^{2 i r w(y)} \frac{y \phi(2 r y)}{\left(1-y^{2}\right)^{\frac{7}{4}}} d y\right|+r^{-1}\left|\int_{\frac{a}{4(x+T) r}}^{\frac{a}{2(x-T) r}} e^{2 i r w(y)} \frac{r \phi^{\prime}(2 r y)}{\left(1-y^{2}\right)^{\frac{3}{4}}} d y\right|=: J_{1}+J_{2} .
$$

As above, Lemma 6.4 gives

$$
J_{1} \ll r^{-2}\left|\int_{\frac{a}{4(x+T) r}}^{\frac{a}{2(x-T) r}}\left(e^{2 i r w(y)}\right)^{\prime} \frac{y^{2} \phi(2 r y)}{\left(1-y^{2}\right)^{\frac{9}{4}}} d y\right| \ll r^{-2} .
$$

For $J_{2}$, we apply Lemma 6.4 with the estimates $\phi^{\prime}(2 r y) \ll x^{2} / a T$ and $y \ll a / r x$ to obtain

$$
J_{2}=r^{-1}\left|\int_{\frac{a}{4(x+T) r}}^{\frac{a}{2(x-T) r}}\left(e^{2 i r w(y)}\right)^{\prime} \frac{y \phi^{\prime}(2 r y)}{\left(1-y^{2}\right)^{\frac{5}{4}}} d y\right| \ll r^{-2} \frac{x}{T} .
$$

So $I_{0} \ll r^{-2} x / T$. This, together with (6.11) and our estimate for $I_{1}$ above, gives (6.8).

\section{Application to sums of Kloosterman sums}

We are in a position to prove Theorem 1.3, which asserts that for $m>0, n<0$ we have

$$
\sum_{c \leq X} \frac{S(m, n, c, \chi)}{c} \ll\left(X^{\frac{1}{6}}+|m n|^{\frac{1}{4}}\right)|m n|^{\epsilon} \log X .
$$

This will follow from an estimate for dyadic sums.

Proposition 7.1. If $m>0, n<0$ and $x>4 \pi \sqrt{\widetilde{m}|\widetilde{n}|}$ then

$$
\sum_{x \leq c \leq 2 x} \frac{S(m, n, c, \chi)}{c} \ll\left(x^{\frac{1}{6}} \log x+|m n|^{\frac{1}{4}}\right)|m n|^{\epsilon}
$$

To obtain Theorem 1.3 from the proposition, we estimate using (2.30) to see that the initial segment $c \leq 4 \pi \sqrt{\widetilde{m}|\widetilde{n}|}$ of $(\underline{7.1})$ contributes $O\left(|m n|^{\frac{1}{4}+\epsilon}\right)$. We break the rest of the sum into dyadic pieces $x \leq c \leq 2 x$ with $4 \pi \sqrt{\widetilde{m}|\widetilde{n}|}<x \leq X / 2$. Estimating each of these with Proposition 7.1 and summing their contributions gives (7.1).

Proof of Proposition 7.1. Let

$$
a:=4 \pi \sqrt{\widetilde{m}|\widetilde{n}|}, \quad T:=x^{\frac{2}{3}},
$$


and suppose that $x>a$. We apply Theorem 4.1 using a test function $\phi$ which satisfies conditions (i)-(iv) of Section 6. Proposition 2.1] and the mean value bound for the divisor function give

$$
\begin{aligned}
\mid \sum_{c=1}^{\infty} \frac{S(m, n, c, \chi)}{c} \phi\left(\frac{a}{c}\right) & -\sum_{\substack{x \leq c \leq 2 x \\
c}} \frac{S(m, n, c, \chi)}{c} \mid \\
& \leq \sum_{\substack{x-T \leq c \leq x \\
2 x \leq c \leq 2}} \frac{|S(m, n, c, \chi)|}{c} \ll \frac{T \log x}{\sqrt{x}}|m n|^{\epsilon} \ll|m n|^{\epsilon} x^{\frac{1}{6}} \log x .
\end{aligned}
$$

Using Theorem 4.1, it will therefore suffice to obtain the stated estimate for the quantity

$$
\sum_{c=1}^{\infty} \frac{S(m, n, c, \chi)}{c} \phi\left(\frac{a}{c}\right)=8 \sqrt{i} \sqrt{\widetilde{m}|\widetilde{n}|} \sum_{1<r_{j}} \frac{\overline{\rho_{j}(m)} \rho_{j}(n)}{\operatorname{ch} \pi r_{j}} \check{\phi}\left(r_{j}\right),
$$

where we have used Corollary 5.3 and the fact that the eigenvalue $r_{0}=i / 4$ makes no contribution since $n$ is negative.

We break the sum (7.3) into dyadic intervals $A \leq r_{j} \leq 2 A$. Using the Cauchy-Schwarz inequality together with Theorems 1.5 and 6.1 (recall that $a / x<1<r_{j}$ ) we obtain

$$
\begin{aligned}
& \sqrt{\widetilde{m}|\widetilde{n}|} \sum_{A \leq r_{j} \leq 2 A}\left|\frac{\overline{\rho_{j}(m)} \rho_{j}(n)}{\operatorname{ch} \pi r_{j}} \check{\phi}\left(r_{j}\right)\right| \\
& \ll \min \left(A^{-\frac{3}{2}}, A^{-\frac{5}{2}} x^{\frac{1}{3}}\right)\left(\widetilde{m} \sum_{A \leq r_{j} \leq 2 A} \frac{\left|\rho_{j}(m)\right|^{2}}{\operatorname{ch} \pi r_{j}}\right)^{\frac{1}{2}}\left(|\widetilde{n}| \sum_{A \leq r_{j} \leq 2 A} \frac{\left|\rho_{j}(n)\right|^{2}}{\operatorname{ch} \pi r_{j}}\right)^{\frac{1}{2}} \\
& \ll \min \left(A^{-\frac{3}{2}}, A^{-\frac{5}{2}} x^{\frac{1}{3}}\right)\left(A^{\frac{3}{2}}+m^{\frac{1}{2}+\epsilon}\right)^{\frac{1}{2}}\left(A^{\frac{5}{2}}+|n|^{\frac{1}{2}+\epsilon} A^{\frac{1}{2}}\right)^{\frac{1}{2}} \\
& \ll \min \left(A^{\frac{1}{2}}, A^{-\frac{1}{2}} x^{\frac{1}{3}}\right)\left(1+m^{\frac{1}{4}+\epsilon} A^{-\frac{3}{4}}+|n|^{\frac{1}{4}+\epsilon} A^{-1}+|m n|^{\frac{1}{4}+\epsilon} A^{-\frac{7}{4}}\right) .
\end{aligned}
$$

Summing the contribution from the dyadic intervals gives

$$
\sqrt{\widetilde{m}|\widetilde{n}|} \sum_{1<r_{j}} \frac{\overline{\rho_{j}(m)} \rho_{j}(n)}{\operatorname{ch} \pi r_{j}} \check{\phi}\left(r_{j}\right) \ll x^{\frac{1}{6}}+|m n|^{\frac{1}{4}+\epsilon},
$$

and Proposition 7.1 follows.

\section{A second estimate For COEFficients of MAAss Cusp Forms}

In the case $m=1$ we can improve the estimate of Proposition 7.1 by using a second estimate for the sum of the Fourier coefficients of Maass cusp forms in $\mathcal{S}_{\frac{1}{2}}(1, \chi)$. The next theorem is an improvement on Theorem 1.5 only when $n$ is much larger than $x$.

Theorem 8.1. Suppose that $\left\{u_{j}\right\}$ is an orthonormal basis of $\mathcal{S}_{\frac{1}{2}}(1, \chi)$ with spectral parameters $r_{j}$ and coefficients $\rho_{j}(n)$ as in (2.23). Suppose that the $u_{j}$ are eigenforms of the Hecke operators $T_{p^{2}}$ for $p \nmid 6$. If $24 n-23$ is not divisible by $5^{4}$ or $7^{4}$ then

$$
\sum_{0<r_{j} \leq x} \frac{\left|\rho_{j}(n)\right|^{2}}{\operatorname{ch} \pi r_{j}} \ll|n|^{-\frac{4}{7}+\epsilon} x^{5-\frac{\operatorname{sgn} n}{2}} .
$$


Remark. As the proof will show, the exponent 4 in the assumption on $n$ in Theorem 8.1 can be replaced by any positive integer $m$. Increasing $m$ has the effect of increasing the implied constant in (8.1).

We require an average version of an estimate of Duke [D, Theorem 5]. Let $\nu_{\theta}$ denote the weight $1 / 2$ multiplier for $\Gamma_{0}(4)$ defined in (2.10).

Proposition 8.2. Let $D$ be an even fundamental discriminant, let $N$ be a positive integer with $D \mid N$, and let

$$
(k, \nu)=\left(\frac{1}{2}, \nu_{\theta}\left(\frac{|D|}{\bullet}\right)\right) \quad \text { or } \quad\left(\frac{3}{2}, \bar{\nu}_{\theta}\left(\frac{|D|}{\bullet}\right)\right) .
$$

Suppose that $\left\{v_{j}\right\}$ is an orthonormal basis of $\mathcal{S}_{k}(N, \nu)$ with spectral parameters $r_{j}$ and coefficients $b_{j}(n)$. If $n>0$ is squarefree then

$$
\sum_{0 \leq r_{j} \leq x} \frac{\left|b_{j}(n)\right|^{2}}{\operatorname{ch} \pi r_{j}} \ll_{N, D} n^{-\frac{4}{7}+\epsilon} x^{5-k}
$$

Sketch of proof. Let $\phi, \widehat{\phi}$ be as in the proof of Theorem 5 of [D]. Then $\widehat{\phi}(r)>0$ and

$$
\widehat{\phi}(r) \sim \frac{1}{2 \pi^{2}}|r|^{k-5} \quad \text { as }|r| \rightarrow \infty .
$$

Set

$$
V_{1}(n, n):=n \sum_{r_{j}} \frac{\left|b_{j}(n)\right|^{2}}{\operatorname{ch} \pi r_{j}} \widehat{\phi}\left(r_{j}\right) .
$$

By Theorem 2 of [D] we have $\left|V_{1}(n, n)+V_{2}(n, n)\right| \ll\left|S_{N}\right|+\left|V_{3}(n, n)\right|$, where $S_{N}$ is defined in the proof of Theorem 5 and $V_{2}, V_{3}$ are defined in Section 3 of that paper. Since $V_{1}(n, n)$ and $V_{2}(n, n)$ are visibly non-negative, we have

$$
V_{1}(n, n) \ll\left|S_{N}\right|+\left|V_{3}(n, n)\right| .
$$

The terms $S_{N}$ and $V_{3}(n, n)$ are estimated by averaging over the level. For $P>(4 \log 2 n)^{2}$ let

$$
\bar{Q}=\{p N: p \text { prime, } P<p \leq 2 P, p \nmid 2 n\} \text {. }
$$

Summing (8.4) gives

$$
\sum_{Q \in \bar{Q}} V_{1}^{(Q)}(n, n) \ll \sum_{Q \in \bar{Q}}\left|S_{Q}\right|+\sum_{Q \in \bar{Q}}\left|V_{3}^{(Q)}(n, n)\right|,
$$

where $V_{1}^{(Q)}, S_{Q}$, and $V_{3}^{(Q)}$ are the analogues of $V_{1}, S_{N}$, and $V_{3}$ for $\Gamma_{0}(Q)$.

For each $Q \in \bar{Q}$, the functions $\left\{\left[\Gamma_{0}(N): \Gamma_{0}(Q)\right]^{-1 / 2} u_{j}(\tau)\right\}$ form an orthonormal subset of $\mathcal{S}_{k}(Q, \nu)$. It follows that

$$
V_{1}^{(Q)}(n, n) \geq \frac{n}{\left[\Gamma_{0}(N): \Gamma_{0}(Q)\right]} \sum_{r_{j}} \frac{\left|b_{j}(n)\right|^{2}}{\operatorname{ch} \pi r_{j}} \widehat{\phi}\left(r_{j}\right)=\frac{V_{1}(n, n)}{\left[\Gamma_{0}(N): \Gamma_{0}(Q)\right]} .
$$

Since $\left[\Gamma_{0}(N): \Gamma_{0}(Q)\right] \leq p+1 \ll P$ we find that

$$
V_{1}(n, n) \ll P V_{1}^{(Q)}(n, n) \quad \text { for all } Q \in \bar{Q} \text {. }
$$


Since $|\bar{Q}| \asymp P / \log P$ we conclude that

$$
V_{1}(n, n) \ll \log P \sum_{Q \in \bar{Q}} V_{1}^{(Q)}(n, n) .
$$

In the proof of Theorem 5 of [D], Duke gives the estimates

$$
\sum_{Q \in \bar{Q}}\left|V_{3}^{(Q)}(n, n)\right| \ll_{N, D} n^{\frac{3}{7}+\epsilon} \quad \text { and } \quad \sum_{Q \in \bar{Q}}\left|S_{Q}\right| \ll_{N, D}\left((n / P)^{\frac{1}{2}}+(n P)^{\frac{3}{8}}\right) n^{\epsilon}
$$

which follow from work of Iwaniec [11]. By (8.5), (8.6), and (8.7) we conclude that

$$
V_{1}(n, n) \ll_{N, D} \log P\left((n / P)^{\frac{1}{2}}+(n P)^{\frac{3}{8}}+n^{\frac{3}{7}}\right) n^{\epsilon} .
$$

Choosing $P=n^{1 / 7}$, we find that $V_{1}(n, n) \ll_{N, D} n^{3 / 7+\epsilon}$. By (8.3) we have

$$
n \sum_{0 \leq r_{j} \leq x} \frac{\left|b_{j}(n)\right|^{2}}{\operatorname{ch} \pi r_{j}} \ll n \sum_{0 \leq r_{j} \leq x} r_{j}^{5-k} \frac{\left|b_{j}(n)\right|^{2}}{\operatorname{ch} \pi r_{j}} \widehat{\phi}\left(r_{j}\right) \ll x^{5-k} V_{1}(n, n),
$$

from which (8.2) follows.

We turn to the proof of Theorem 8.1 ,

Proof of Theorem 8.1. Set $\alpha:=\left[\Gamma_{0}(1): \Gamma_{0}(24,24)\right]^{1 / 2}$. With $\left\{u_{j}\right\}$ as in the hypotheses, and recalling (2.21), define

$$
\begin{aligned}
& v_{j}(\tau):=\frac{1}{\alpha} u_{j}(24 \tau)=\sum_{n \equiv 1(24)} b_{j}(n) W_{\frac{1}{4} \operatorname{sgn} n, i r_{j}}(4 \pi|n| y) e(n x), \\
& v_{j}^{\prime}(\tau):=\frac{1}{\alpha}\left(r_{j}^{2}+\frac{1}{16}\right)^{-\frac{1}{2}} \overline{L_{\frac{1}{2}} u_{j}}(24 \tau)=\sum_{n \equiv 23(24)} b_{j}^{\prime}(n) W_{\frac{3}{4} \operatorname{sgn} n, i r_{j}}(4 \pi|n| y) e(n x) .
\end{aligned}
$$

By (2.11) we have

$$
v_{j} \in \mathcal{S}_{\frac{1}{2}}\left(576, \nu_{\theta}\left(\frac{12}{\bullet}\right)\right), \quad v_{j}^{\prime} \in \mathcal{S}_{\frac{3}{2}}\left(576, \overline{\nu_{\theta}}\left(\frac{12}{\bullet}\right)\right) .
$$

From (2.18) and (2.21) we see that $\left\{v_{j}\right\},\left\{v_{j}^{\prime}\right\}$ are orthonormal sets which can be extended to orthonormal bases for these spaces. Using (2.16) and (2.17) and comparing Fourier expansions, we find for positive $n$ that

$$
b_{j}(n)=\frac{1}{\alpha} \rho_{j}\left(\frac{n+23}{24}\right), \quad b_{j}^{\prime}(n)=\frac{1}{\alpha}\left(r_{j}^{2}+\frac{1}{16}\right)^{-\frac{1}{2}} \overline{\rho_{j}\left(\frac{-n+23}{24}\right)} .
$$

It follows from Proposition 8.2 that for squarefree $n$ we have

$$
\sum_{0<r_{j} \leq x} \frac{\left|\rho_{j}\left(\frac{n+23}{24}\right)\right|^{2}}{\operatorname{ch} \pi r_{j}} \ll|n|^{-\frac{4}{7}+\epsilon} x^{5-\frac{\operatorname{sgn} n}{2}} .
$$

To establish (8.8) for non-squarefree $n$ we use the assumption that the $\left\{u_{j}\right\}$ are Hecke eigenforms. Recall the definition of the lift $S_{t}$ from Theorem 5.1. For each $j$ there exists some squarefree positive $t \equiv 1(\bmod 24)$ for which $S_{t}\left(u_{j}\right) \neq 0$. Denote by $\lambda_{j}(p)$ the eigenvalue of $u_{j}$ under $T_{p^{2}}$. From Corollary 5.2 it follows that $\left(\frac{12}{p}\right) \lambda_{j}(p)$ is a Hecke eigenvalue of the weight zero Maass cusp form $S_{t}\left(u_{j}\right)$. For these eigenvalues we have the estimate

$$
\left|\lambda_{j}(p)\right| \leq p^{\frac{7}{64}}+p^{-\frac{7}{64}}
$$

due to Kim and Sarnak [Ki, Appendix 2]. 
The Hecke action (2.24) gives

$$
p \rho_{j}\left(\frac{p^{2} n+23}{24}\right)=\lambda_{j}(p) \rho_{j}\left(\frac{n+23}{24}\right)-p^{-\frac{1}{2}}\left(\frac{12 n}{p}\right) \rho_{j}\left(\frac{n+23}{24}\right)-p^{-1} \rho_{j}\left(\frac{n / p^{2}+23}{24}\right) .
$$

Suppose that $p^{2} \nmid n$. Then

$$
\left|\rho_{j}\left(\frac{p^{2} n+23}{24}\right)\right| \leq\left(p^{-\frac{57}{64}}+p^{-\frac{71}{64}}+p^{-\frac{3}{2}}\right)\left|\rho_{j}\left(\frac{n+23}{24}\right)\right| \leq \begin{cases}1.25 p^{-\frac{4}{7}} & \text { if } p=5 \text { or } 7 \\ p^{-\frac{4}{7}} & \text { if } p \geq 11 .\end{cases}
$$

Thus (8.8) holds whenever $n$ is not divisible by $p^{4}$ for any $p$.

To treat the remaining cases, assume that $p \geq 11$ and that for $p^{2} \nmid n$ we have

$$
\left|\rho_{j}\left(\frac{p^{2 \ell} n+23}{24}\right)\right| \leq p^{-\frac{4 \ell}{7}}\left|\rho_{j}\left(\frac{n+23}{24}\right)\right|, \quad \ell \leq m-1 .
$$

Then for $m \geq 2$, the relation (8.9) gives

$$
\begin{aligned}
\left|\rho_{j}\left(\frac{p^{2 m} n+23}{24}\right)\right| & \leq\left(p^{-\frac{57}{64}}+p^{-\frac{71}{64}}\right)\left|\rho_{j}\left(\frac{p^{2 m-2} n+23}{24}\right)\right|+p^{-2}\left|\rho_{j}\left(\frac{p^{2 m-4} n+23}{24}\right)\right| \\
& \leq\left[\left(p^{-\frac{57}{64}}+p^{-\frac{71}{64}}\right) p^{\frac{4}{7}}+p^{-2+\frac{8}{7}}\right] p^{-\frac{4 m}{7}}\left|\rho_{j}\left(\frac{n+23}{24}\right)\right| .
\end{aligned}
$$

For $p \geq 11$ the quantity in brackets is $<1$. It follows that (8.8) holds for all $n$ which are not divisible by $5^{4}$ or $7^{4}$.

\section{Sums of Kloosterman sums in the Case $m=1$}

Throughout this section, $n$ will denote a negative integer. In our application to the error term in Rademacher's formula we need a estimate for sums of the Kloosterman sums $S(1, n, c, \chi)$ which improves the bound of Theorem 1.3 with respect to $n$. We will also make the assumption throughout that

$$
24 n-23 \text { is not divisible by } 5^{4} \text { or } 7^{4}
$$

so that we may apply Theorem 8.1 (see the remark after that theorem).

Theorem 9.1. For $0<\delta<1 / 2$ and $n<0$ satisfying (9.1) we have

$$
\sum_{c \leq X} \frac{S(1, n, c, \chi)}{c} \ll_{\delta}|n|^{\frac{13}{56}+\epsilon} X^{\frac{3}{4} \delta}+\left(|n|^{\frac{41}{168}+\epsilon}+X^{\frac{1}{2}-\delta}\right) \log X .
$$

Theorem 9.1 will follow from an estimate for dyadic sums.

Proposition 9.2. Suppose that $0<\delta<1 / 2$ and that $n<0$ satisfies (9.1). Then

$$
\sum_{x \leq c \leq 2 x} \frac{S(1, n, c, \chi)}{c} \ll_{\delta}|n|^{\frac{41}{28}+\epsilon} x^{-\frac{5}{2}}+|n|^{\frac{13}{56}+\epsilon} x^{\frac{3}{4} \delta}+x^{\frac{1}{2}-\delta} \log x .
$$

Deduction of Theorem 9.1 from Proposition 9.2. We break the sum (9.2) into an initial segment corresponding to $c \leq|n|^{\alpha}$ and dyadic intervals of the form (9.3) with $x \geq|n|^{\alpha}$. Estimating the initial segment using Lehmer's bound (1.4) and applying Proposition 9.2 to each of the dyadic intervals, we find that

$$
\sum_{c \leq X} \frac{S(1, n, c, \chi)}{c} \ll_{\delta, \alpha}|n|^{\frac{\alpha}{2}+\epsilon}+|n|^{\frac{13}{56}+\epsilon} X^{\frac{3}{4} \delta}+\left(|n|^{\frac{41}{28}-\frac{5}{2} \alpha+\epsilon}+X^{\frac{1}{2}-\delta}\right) \log X .
$$


Theorem 9.1 follows upon setting $\alpha=41 / 84$.

Proof of Proposition 9.2. Let $T$ satisfy

$$
T \asymp x^{1-\delta} \quad \text { and } \quad T \leq \frac{x}{3},
$$

where $0<\delta<1 / 2$ is a parameter to be chosen later, and set

$$
a:=4 \pi \sqrt{|\widetilde{n}|} .
$$

Arguing as in (7.2) (using Lehmer's bound (1.4) to remove the dependence on $n$ ) we have

$$
\left|\sum_{c=1}^{\infty} \frac{S(1, n, c, \chi)}{c} \phi\left(\frac{a}{c}\right)-\sum_{x \leq c \leq 2 x} \frac{S(1, n, c, \chi)}{c}\right| \ll \delta x^{\frac{1}{2}-\delta} \log x .
$$

Let $\left\{u_{j}\right\}$ be an orthonormal basis for $\mathcal{S}_{\frac{1}{2}}(1, \chi)$ as in (2.23) which consists of eigenforms for the Hecke operators $T_{p^{2}}$ with $p \nmid 6$. Theorem 4.1 gives

$$
\sum_{c=1}^{\infty} \frac{S(1, n, c, \chi)}{c} \phi\left(\frac{a}{c}\right)=8 \sqrt{i} \sqrt{|\widetilde{n}|} \sum_{r_{j}} \frac{\overline{\rho_{j}(1)} \rho_{j}(n)}{\operatorname{ch} \pi r_{j}} \check{\phi}\left(r_{j}\right) .
$$

We break the sum on $r_{j}$ into ranges corresponding to the three ranges of the $K$-Bessel function:
(i) $r_{j} \leq \frac{a}{8 x}$
(ii) $\frac{a}{8 x}<r_{j}<\frac{a}{x}$,
(iii) $r_{j} \geq \frac{a}{x}$.

We may restrict our attention to $r_{j}>1$ by Corollary 5.3 (the eigenvalue $r_{0}=i / 4$ does not contribute).

For the first range, Theorem 6.1 gives gives $\check{\phi}\left(r_{j}\right) \ll r_{j}^{-3 / 2} e^{-r_{j} / 2}$. By Theorem 8.1 we have

$$
\frac{\rho_{j}(1)}{\sqrt{\operatorname{ch} \pi r_{j}}} \ll r_{j}^{\frac{9}{4}}, \quad \frac{\rho_{j}(n)}{\sqrt{\operatorname{ch} \pi r_{j}}} \ll|n|^{-\frac{2}{7}+\epsilon} r_{j}^{\frac{11}{4}} .
$$

Combining these estimates gives

$$
\sqrt{|\widetilde{n}|} \sum_{1<r_{j} \leq \frac{a}{8 x}}\left|\frac{\overline{\rho_{j}(1)} \rho_{j}(n)}{\operatorname{ch} \pi r_{j}} \check{\phi}\left(r_{j}\right)\right| \ll|n|^{\frac{3}{14}+\epsilon} \sum_{1<r_{j} \leq \frac{a}{8 x}} r_{j}^{\frac{7}{2}} e^{-r_{j} / 2} \ll|n|^{\frac{3}{14}+\epsilon},
$$

where we have used (2.22) to conclude that the latter sum over $r_{j}$ is $O(1)$.

For the other ranges we need the mean value estimates of Theorem 1.5 .

$$
\begin{gathered}
\sum_{1<r_{j} \leq x} \frac{\left|\rho_{j}(1)\right|^{2}}{\operatorname{ch} \pi r_{j}} \ll x^{\frac{3}{2}}, \\
|\widetilde{n}| \sum_{1<r_{j} \leq x} \frac{\left|\rho_{j}(n)\right|^{2}}{\operatorname{ch} \pi r_{j}} \ll x^{\frac{5}{2}}+|n|^{\frac{1}{2}+\epsilon} x^{\frac{1}{2}},
\end{gathered}
$$


as well as the average version of Duke's estimate (Theorem 8.1):

$$
|\widetilde{n}| \sum_{r_{j} \leq x} \frac{\left|\rho_{j}(n)\right|^{2}}{\operatorname{ch} \pi r_{j}} \ll|n|^{\frac{3}{7}+\epsilon} x^{\frac{11}{2}} .
$$

Using (9.7) and (9.8) with the Cauchy-Schwarz inequality, we find that

$$
\sqrt{|\widetilde{n}|} \sum_{r_{j} \leq x} \frac{\left|\overline{\rho_{j}(1)} \rho_{j}(n)\right|}{\operatorname{ch} \pi r_{j}} \ll x^{\frac{3}{4}}\left(x^{\frac{5}{2}}+|n|^{\frac{1}{2}+\epsilon} x^{\frac{1}{2}}\right)^{\frac{1}{2}} \ll x^{2}+|n|^{\frac{1}{4}+\epsilon} x .
$$

Using (9.7) and (9.9) we obtain

$$
\sqrt{|\widetilde{n}|} \sum_{r_{j} \leq x} \frac{\left|\overline{\rho_{j}(1)} \rho_{j}(n)\right|}{\operatorname{ch} \pi r_{j}} \ll x^{\frac{3}{4}}\left(|n|^{\frac{3}{7}+\epsilon} x^{\frac{11}{2}}\right)^{\frac{1}{2}} \ll|n|^{\frac{3}{14}+\epsilon} x^{\frac{7}{2}} .
$$

In the second range Theorem 6.1 gives $\check{\phi}\left(r_{j}\right) \ll r_{j}^{-1}$. It follows from (9.10) (assuming as we may that $a / x \geq 1$ ) that

$$
\sqrt{|\widetilde{n}|} \sum_{\frac{a}{8 x}<r_{j}<\frac{a}{x}}\left|\frac{\overline{\rho_{j}(1)} \rho_{j}(n)}{\operatorname{ch} \pi r_{j}} \check{\phi}\left(r_{j}\right)\right| \ll|n|^{\frac{3}{14}+\epsilon}\left(\frac{a}{x}\right)^{\frac{5}{2}} \ll|n|^{\frac{41}{28}+\epsilon} x^{-\frac{5}{2}} .
$$

In the third range Theorem 6.1 gives

$$
\check{\phi}\left(r_{j}\right) \ll \min \left(r_{j}^{-\frac{3}{2}}, r_{j}^{-\frac{5}{2}} \frac{x}{T}\right) .
$$

We use dyadic sums corresponding to intervals $A \leq r_{j} \leq 2 A$ with $A \geq \max \left(\frac{a}{x}, 1\right)$. For such a sum we have

$$
\begin{aligned}
\sqrt{|\widetilde{n}|} \sum_{A \leq r_{j} \leq 2 A}\left|\frac{\overline{\rho_{j}(1)} \rho_{j}(n)}{\operatorname{ch} \pi r_{j}} \check{\phi}\left(r_{j}\right)\right| & \ll \min \left(A^{-\frac{3}{2}}, A^{-\frac{5}{2}} \frac{x}{T}\right) \min \left(A^{2}+|n|^{\frac{1}{4}+\epsilon} A,|n|^{\frac{3}{14}+\epsilon} A^{\frac{7}{2}}\right) \\
& \ll \min \left(A^{\frac{1}{2}}, A^{-\frac{1}{2}} \frac{x}{T}\right)\left(1+|n|^{\frac{13}{56}+\epsilon} A^{\frac{1}{4}}\right) \\
& \ll|n|^{\frac{13}{56}+\epsilon} \min \left(A^{\frac{3}{4}}, A^{-\frac{1}{4}} \frac{x}{T}\right),
\end{aligned}
$$

where we have used the fact that for positive $B, C$, and $D$ we have

$$
\min (B, C+D) \leq \min (B, C)+\min (C, D) \quad \text { and } \quad \min (B, C) \leq \sqrt{B C} .
$$

Combining the dyadic sums, we find that

$$
\sqrt{|\widetilde{n}|} \sum_{r_{j} \geq \frac{a}{x}}\left|\frac{\overline{\rho_{j}(1)} \rho_{j}(n)}{\operatorname{ch} \pi r_{j}} \check{\phi}\left(r_{j}\right)\right| \ll|n|^{\frac{13}{56}+\epsilon}\left(\frac{x}{T}\right)^{\frac{3}{4}} \ll_{\delta}|n|^{\frac{13}{56}+\epsilon} x^{\frac{3}{4} \delta} .
$$

Using (9.5) with (9.6), (9.11), and (9.12) we obtain

$$
\sum_{c=1}^{\infty} \frac{S(1, n, c, \chi)}{c} \check{\phi}\left(\frac{a}{c}\right) \ll_{\delta}|n|^{\frac{41}{28}+\epsilon} x^{-\frac{5}{2}}+|n|^{\frac{13}{56}+\epsilon} x^{\frac{3}{4} \delta} .
$$

Proposition 9.2 follows from this estimate together with (9.4). 


\section{Application to the REMAinder term in RADEMACHER'S FORMula}

In the final section, we use Theorem 9.1 to prove Theorems 1.1 and 1.2 . Recall that we wish to bound $R(n, N)$, where

$$
p(n)=\frac{2 \pi}{(24 n-1)^{\frac{3}{4}}} \sum_{c=1}^{N} \frac{A_{c}(n)}{c} I_{\frac{3}{2}}\left(\frac{\pi \sqrt{24 n-1}}{6 c}\right)+R(n, N),
$$

and

$$
A_{c}(n)=\sqrt{-i} S(1,1-n, c, \chi) .
$$

To match the notation of the previous sections, we assume that $n<0$ satisfies (9.1) and we provide a bound for

$$
R(1-n, N)=\frac{2 \pi \sqrt{-i}}{24^{\frac{3}{4}}}|\widetilde{n}|^{-\frac{3}{4}} \sum_{c>N} \frac{S(1, n, c, \chi)}{c} I_{\frac{3}{2}}\left(\frac{a}{c}\right),
$$

where

$$
a:=\sqrt{\frac{2}{3}} \pi \sqrt{|\widetilde{n}|}
$$

We will apply partial summation to (10.1). For fixed $\nu, M>0$ and $0 \leq z \leq M$, the asymptotic formula [DL, (10.30.1)] gives

$$
I_{\nu}(z) \ll_{\nu, M} z^{\nu}
$$

We have a straightforward lemma.

Lemma 10.1. Suppose that $a>0$ and that $\alpha>0$. Then for $\frac{t}{a} \geq \alpha$ we have

$$
\left(I_{\frac{3}{2}}(a / t)\right)^{\prime} \ll_{\alpha} a^{\frac{3}{2}} t^{-\frac{5}{2}}
$$

Proof. By [DL, (10.29.1)] we have

$$
I_{\frac{3}{2}}^{\prime}(t)=\frac{1}{2}\left(I_{\frac{1}{2}}(t)+I_{\frac{5}{2}}(t)\right) .
$$

It follows that

$$
\left(I_{\frac{3}{2}}(a / t)\right)^{\prime}=-\frac{a}{2 t^{2}}\left(I_{\frac{1}{2}}(a / t)+I_{\frac{5}{2}}(a / t)\right) .
$$

For $\frac{t}{a} \geq \alpha$, equations (10.3) and (10.4) give

$$
\left(I_{\frac{3}{2}}(a / t)\right)^{\prime} \ll_{\alpha} a^{\frac{3}{2}} t^{-\frac{5}{2}}+a^{\frac{7}{2}} t^{-\frac{9}{2}},
$$

and the lemma follows.

Proof of Theorems 1.1 and 1.2. Let $a$ be as in (10.2). Then

$$
R(1-n, N) \ll|\widetilde{n}|^{-\frac{3}{4}}\left|\sum_{c>N} \frac{S(1, n, c, \chi)}{c} I_{\frac{3}{2}}\left(\frac{a}{c}\right)\right| .
$$

Let

$$
S(n, X):=\sum_{c \leq X} \frac{S(1, n, c, \chi)}{c}
$$

so that Theorem 9.1 (for $0<\delta<1 / 2$ ) gives

$$
S(n, X) \ll_{\delta}|n|^{\frac{13}{56}+\epsilon} X^{\frac{3}{4} \delta}+\left(|n|^{\frac{41}{168}+\epsilon}+X^{\frac{1}{2}-\delta}\right) \log X .
$$


By partial summation using (10.3) we have

$$
\sum_{c>N} \frac{S(1, n, c, \chi)}{c} I_{\frac{3}{2}}\left(\frac{a}{c}\right)=-S(n, N) I_{\frac{3}{2}}(a / N)-\int_{N}^{\infty} S(n, t)\left(I_{\frac{3}{2}}(a / t)\right)^{\prime} d t .
$$

Let $\alpha>0$ be fixed and take

$$
N=\alpha|n|^{\frac{1}{2}+\beta}
$$

where $\beta \in[0,1 / 2]$ is to be chosen. By (10.3) we have

$$
I_{\frac{3}{2}}(a / N) \ll_{\alpha}|n|^{\frac{3}{4}} N^{-\frac{3}{2}}
$$

and by Lemma 10.1 we have

$$
\left(I_{\frac{3}{2}}(a / t)\right)^{\prime} \ll_{\alpha}|n|^{\frac{3}{4}} t^{-\frac{5}{2}} \quad \text { for } t \geq N .
$$

Thus

$$
S(n, N) I_{\frac{3}{2}}(a / N) \ll_{\delta, \alpha}|n|^{\frac{3}{4}} N^{-\frac{3}{2}}\left(|n|^{\frac{13}{56}} N^{\frac{3}{4} \delta}+|n|^{\frac{41}{168}}+N^{\frac{1}{2}-\delta}\right)|n|^{\epsilon},
$$

and the same bound holds for the integral term. Therefore

$$
R\left(1-n, \alpha|n|^{\frac{1}{2}+\beta}\right) \ll_{\delta, \alpha}|n|^{\left(\frac{1}{2}+\beta\right)\left(\frac{3}{4} \delta-\frac{3}{2}\right)+\frac{13}{56}+\epsilon}+|n|^{-\frac{3}{2}\left(\frac{1}{2}+\beta\right)+\frac{41}{168}+\epsilon}+|n|^{-\left(\frac{1}{2}+\beta\right)(1+\delta)+\epsilon} .
$$

When $\beta=0$, the estimate (10.5) becomes

$$
R\left(1-n, \alpha|n|^{\frac{1}{2}}\right) \ll_{\delta, \alpha}|n|^{\frac{3}{8} \delta-\frac{29}{56}+\epsilon}+|n|^{-\frac{85}{168}+\epsilon}+|n|^{-\frac{1}{2}-\frac{1}{2} \delta+\epsilon} .
$$

For any choice of $\delta \in\left[\frac{1}{84}, \frac{2}{63}\right]$ this gives

$$
R\left(1-n, \alpha|n|^{\frac{1}{2}}\right) \ll_{\alpha}|n|^{-\frac{1}{2}-\frac{1}{168}+\epsilon} .
$$

Theorem 1.1 follows after replacing $n$ by $1-n$.

To optimize, we choose $\beta=\frac{5}{252}$ and $\delta=\frac{4}{131}$, which makes all three exponents in (10.5) equal to $-\frac{1}{2}-\frac{1}{28}$. This gives Theorem 1.2

\section{REFERENCES}

[A] N. Andersen. Singular invariants and coefficients of weak harmonic Maass forms of weight 5/2. Preprint, arXiv:1410.7349 [math.NT].

[AL] A. O. L. Atkin and J. Lehner. Hecke operators on $\Gamma_{0}(m)$. Math. Ann., 185:134-160, 1970.

[Ba] C. B. Balogh. Uniform asymptotic expansions of the modified Bessel function of the third kind of large imaginary order. Bull. Amer. Math. Soc., 72:40-43, 1966.

[BM] E. M. Baruch and Z. Mao. A generalized Kohnen-Zagier formula for Maass forms. J. Lond. Math. Soc. (2), 82(1):1-16, 2010.

[BO] B. C. Berndt and K. Ono. Ramanujan's unpublished manuscript on the partition and tau functions with proofs and commentary. Sém. Lothar. Combin., 42, 1999. In The Andrews Festschrift, D. Foata and G.-N. Han, eds., Springer-Verlag, Berlin, 2001, pp. 39-110.

[Bl] V. Blomer. Sums of Hecke eigenvalues over values of quadratic polynomials. Int. Math. Res. Not. IMRN, (16):Art. ID rnn059. 29, 2008.

[BS] A. R. Booker and A. Strömbergsson. Numerical computations with the trace formula and the Selberg eigenvalue conjecture. J. Reine Angew. Math., 607:113-161, 2007.

[BST] A. R. Booker, A. Strömbergsson, and H. Then. Bounds and algorithms for the $K$-Bessel function of imaginary order. LMS J. Comput. Math., 16:78-108, 2013.

[Br1] R. W. Bruggeman. Modular forms of varying weight. III. J. Reine Angew. Math., 371:144-190, 1986.

[Br2] R. W. Bruggeman. Kloosterman sums for the modular group. In Number theory in progress, Vol. 2 (Zakopane-Kościelisko, 1997), pages 651-674. de Gruyter, Berlin, 1999. 
[C] B. A. Cipra. On the Niwa-Shintani theta-kernel lifting of modular forms. Nagoya Math. J., 91:49117, 1983.

[DL] NIST Digital Library of Mathematical Functions. http://dlmf.nist.gov/, Release 1.0.10 of 2015-0807. Online companion to OLBC].

[D] W. Duke. Hyperbolic distribution problems and half-integral weight Maass forms. Invent. Math., 92(1):73-90, 1988.

[DFI] W. Duke, J. B. Friedlander, and H. Iwaniec. The subconvexity problem for Artin $L$-functions. Invent. Math., 149(3):489-577, 2002.

[FM] A. Folsom and R. Masri. Equidistribution of Heegner points and the partition function. Math. Ann., 348(2):289-317, 2010.

[GSe] S. Ganguly and J. Sengupta. Sums of Kloosterman sums over arithmetic progressions. Int. Math. Res. Not. IMRN, (1):137-165, 2012.

[GS] D. Goldfeld and P. Sarnak. Sums of Kloosterman sums. Invent. Math., 71(2):243-250, 1983.

[HR] G. H. Hardy and S. Ramanujan. Asymptotic formulæ in combinatory analysis [Proc. London Math. Soc. (2) 17 (1918), 75-115]. In Collected papers of Srinivasa Ramanujan, pages 276-309. AMS Chelsea Publ., Providence, RI, 2000.

[HB] D. R. Heath-Brown. Arithmetic applications of Kloosterman sums. Nieuw Arch. Wiskd. (5), $1(4): 380-384,2000$.

[He] D. A. Hejhal. The Selberg trace formula for $\operatorname{PSL}(2, \mathbb{R})$. Vol. 2. Lecture Notes in Mathematics. Springer-Verlag, Berlin, 1983.

[H1] M. N. Huxley. Introduction to Kloostermania. In Elementary and analytic theory of numbers (Warsaw, 1982), volume 17 of Banach Center Publ., pages 217-306. PWN, Warsaw, 1985.

[H2] M. N. Huxley. Exceptional eigenvalues and congruence subgroups. In The Selberg trace formula and related topics (Brunswick, Maine, 1984), volume 53 of Contemp. Math., pages 341-349. Amer. Math. Soc., Providence, RI, 1986.

[I1] H. Iwaniec. Fourier coefficients of modular forms of half-integral weight. Invent. Math., 87(2):385401, 1987.

[I2] H. Iwaniec. Spectral methods of automorphic forms. Graduate Studies in Mathematics. American Mathematical Society, Providence, RI; Revista Matemática Iberoamericana, Madrid, second edition, 2002.

[KS] S. Katok and P. Sarnak. Heegner points, cycles and Maass forms. Israel J. Math., 84(1-2):193-227, 1993.

[Ki] H. H. Kim. Functoriality for the exterior square of $\mathrm{GL}_{4}$ and the symmetric fourth of $\mathrm{GL}_{2}$. J. Amer. Math. Soc., 16(1):139-183, 2003. With appendix 1 by D. Ramakrishnan and appendix 2 by Kim and P. Sarnak.

[K] M. I. Knopp. Modular functions in analytic number theory. Markham Publishing Co., Chicago, Ill., 1970.

[Kn] I. Knowles. Integral mean value theorems and the Ganelius inequality. Proc. Roy. Soc. Edinburgh Sect. A, 97:145-150, 1984 .

$[\mathrm{Ku}] \quad$ N. V. Kuznetsov. The Petersson conjecture for cusp forms of weight zero and the Linnik conjecture. Sums of Kloosterman sums. Mat. Sb. (N.S.), 111(153):334-383, 1980.

[L1] D. H. Lehmer. On the series for the partition function. Trans. Amer. Math. Soc., 43(2):271-295, 1938.

[L2] D. H. Lehmer. On the remainders and convergence of the series for the partition function. Trans. Amer. Math. Soc., 46:362-373, 1939.

[Li] J. V. Linnik. Additive problems and eigenvalues of the modular operators. In Proc. Internat. Congr. Mathematicians (Stockholm, 1962), pages 270-284. Inst. Mittag-Leffler, Djursholm, 1963.

[LMF] LMFDB Collaboration. The $L$-functions and modular forms database, Maass forms on $\Gamma_{0}(6)$. http://www.lmfdb.org/ModularForm/GL2/Q/Maass, 2013. [Online; accessed 1 October 2015].

[M1] H. Maass. Über eine neue Art von nichtanalytischen automorphen Funktionen und die Bestimmung Dirichletscher Reihen durch Funktionalgleichungen. Math. Ann., 121:141-183, 1949.

[M2] H. Maass. Die Differentialgleichungen in der Theorie der elliptischen Modulfunktionen. Math. Ann., 125:235-263, 1952. 
[N] S. Niwa. Modular forms of half integral weight and the integral of certain theta-functions. Nagoya Math. J., 56:147-161, 1975.

[OLBC] F. W. J. Olver, D. W. Lozier, R. F. Boisvert, and C. W. Clark, editors. NIST Handbook of Mathematical Functions. Cambridge University Press, New York, NY, 2010. Print companion to [DL].

$[\mathrm{Pr}] \quad$ W. d. A. Pribitkin. A generalization of the Goldfeld-Sarnak estimate on Selberg's Kloosterman zeta-function. Forum Math., 12(4):449-459, 2000.

[P1] N. V. Proskurin. The summation formulas for general Kloosterman sums. Zap. Nauchn. Sem. Leningrad. Otdel. Mat. Inst. Steklov. (LOMI), 82:103-135, 1979.

[P2] N. V. Proskurin. On general Kloosterman sums. Zap. Nauchn. Sem. S.-Peterburg. Otdel. Mat. Inst. Steklov. (POMI), 302(19):107-134, 2003.

[R1] H. Rademacher. On the partition function $p(n)$. Proc. London Math. Soc., 43(4):241-254, 1936.

[R2] H. Rademacher. On the expansion of the partition function in a series. Ann. of Math. (2), 44:416$422,1943$.

[R3] H. Rademacher. On the Selberg formula for $A_{k}(n)$. J. Indian Math. Soc. (N.S.), 21:41-55 (1958), 1957.

[R4] H. Rademacher. Topics in analytic number theory. Springer-Verlag, New York-Heidelberg, 1973. Edited by E. Grosswald, J. Lehner and M. Newman, Die Grundlehren der mathematischen Wissenschaften, Band 169.

[Ro1] W. Roelcke. Das Eigenwertproblem der automorphen Formen in der hyperbolischen Ebene, I. Math. Ann., 167:292-337, 1966.

[Ro2] W. Roelcke. Das Eigenwertproblem der automorphen Formen in der hyperbolischen Ebene, II. Math. Ann., 168:261-324, 1966.

[Sa] P. Sarnak. Additive number theory and Maass forms. In Number theory (New York, 1982), volume 1052 of Lecture Notes in Math., pages 286-309. Springer, Berlin, 1984.

[ST] P. Sarnak and J. Tsimerman. On Linnik and Selberg's conjecture about sums of Kloosterman sums. In Algebra, arithmetic, and geometry: in honor of Yu. I. Manin. Vol. II, volume 270 of Progr. Math., pages 619-635. Birkhäuser Boston, Inc., Boston, MA, 2009.

[Se1] A. Selberg. Harmonic analysis and discontinuous groups in weakly symmetric Riemannian spaces with applications to Dirichlet series. J. Indian Math. Soc. (N.S.), 20:47-87, 1956.

[Se2] A. Selberg. On the estimation of Fourier coefficients of modular forms. In Proc. Sympos. Pure Math., Vol. VIII, pages 1-15. Amer. Math. Soc., Providence, R.I., 1965.

[S] G. Shimura. On modular forms of half integral weight. Ann. of Math. (2), 97:440-481, 1973.

[Sh] T. Shintani. On construction of holomorphic cusp forms of half integral weight. Nagoya Math. J., $58: 83-126,1975$.

[T] E. C. Titchmarsh. The Theory of the Riemann Zeta-Function. Oxford, at the Clarendon Press, 1951.

[W] A. Weil. On some exponential sums. Proc. Nat. Acad. Sci. U. S. A., 34:204-207, 1948.

[Wh] A. L. Whiteman. A sum connected with the series for the partition function. Pacific J. Math., 6:159-176, 1956.

Department of Mathematics, University of Illinois, Urbana, IL 61801

E-mail address: sahlgren@illinois.edu

Department of Mathematics, University of Illinois, Urbana, IL 61801

E-mail address: nandrsn4@illinois.edu 